\title{
Integrated Optical Sensors utilizing Slow-light Propagation in Grated-waveguide Cavities
}




\section{Graduation committee}

\section{Chairman and secretary}

Prof. Dr. Ir. A. J. Mouthaan University of Twente

\section{Promoter}

Prof. Dr. M. Pollnau University of Twente

\section{Assistant promoter}

Dr. H. J. W. M. Hoekstra University of Twente

\section{Members}

$\begin{array}{ll}\text { Prof. Dr. Ir. G. J. M. Krijnen } & \text { University of Twente } \\ \text { Prof. Dr. S. G. Lemay } & \text { University of Twente } \\ \text { Prof. Dr. Ir. D. Van Thourhout } & \text { Ghent University } \\ \text { Dr. Ir. R. M. de Ridder } & \text { University of Twente }\end{array}$

The research described in this thesis was carried out at the Integrated Optical MicroSystems (IOMS) Group, Faculty of Electrical Engineering, Mathematics and Computer Science, MESA+ Institute for Nanotechnology, University of Twente, P.O. Box 217, 7500 AE, Enschede, The Netherlands. It was financially supported by MEMSland, a project of the Point One program funded by the Dutch Ministry of Economic Affairs and the Dutch Technology Foundation - STW through project TOE. 6596.

Front cover: 3D schematic picture of a grated-waveguide (GWG), and its transmission spectra.

Back cover: SEM image of a GWG-cantilever integrated device.

Printed by Wöhrmann Print Service, Zutphen, The Netherlands.

Copyright $@ 2012$ by PHAM VAN SO, Veldhoven, The Netherlands. All rights reserved.

ISBN 978-90-365-3373-7

DOI: $10.3990 / 1.9789036533737$

URL: http://dx.doi.org/10.3990/1.9789036533737 


\title{
INTEGRATED OPTICAL SENSORS \\ UTILIZING SLOW-LIGHT PROPAGATION \\ IN GRATED-WAVEGUIDE CAVITIES
}

\section{DISSERTATION}

\author{
to obtain \\ the degree of doctor at the University of Twente, \\ on the authority of the rector magnificus, \\ prof. dr. H. Brinksma \\ on account of the decision of the graduation committee, \\ to be publicly defended \\ on Friday the $1^{\text {st }}$ of June 2012 at 12:45
}

by

PHAM VAN SO

born on the $16^{\text {th }}$ of October 1981

in Long An, Vietnam 
This dissertation is approved by:

the promoter: Prof. Dr. M. Pollnau

the assistant promoter: Dr. Hugo J. W. M. Hoekstra 
Pedicated to myparents

LeThiturho sham Van Vimh

Tomigroothers and sisters

Rimh Táng ballávätulchitem

- Pham Vanso - 



\section{Contents}

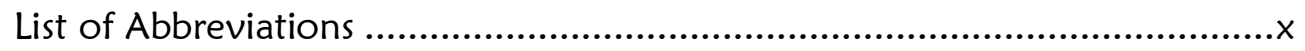

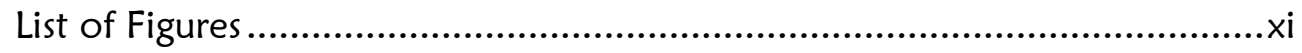

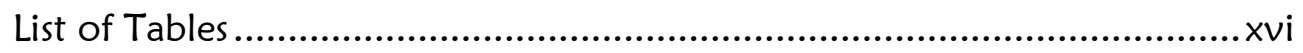

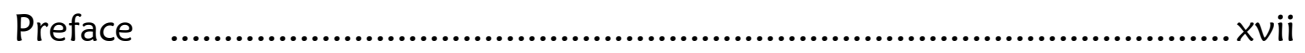

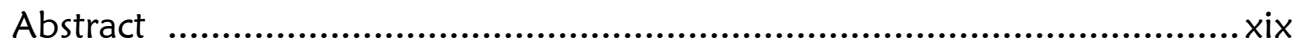

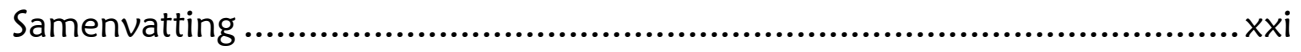

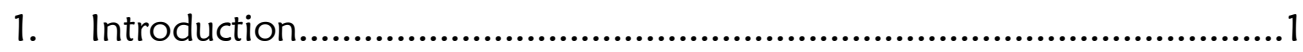

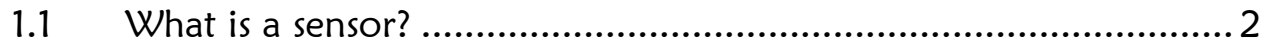

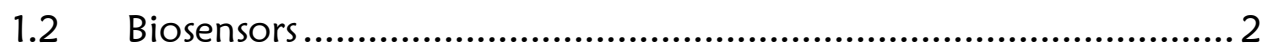

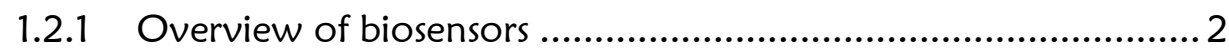

1.2.2 Label-free biosensors based on optical resonant cavities ............. 3

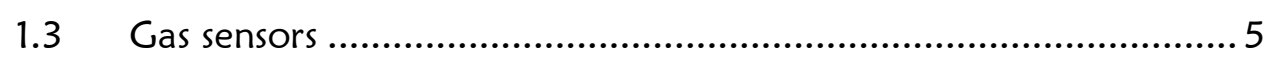

1.3.1 Trace gas sensing methods.................................................... 6

1.3.2 Cantilever-based gas sensors.............................................. 7

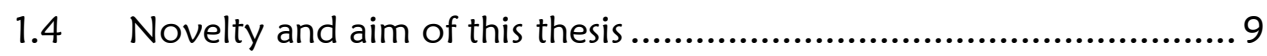

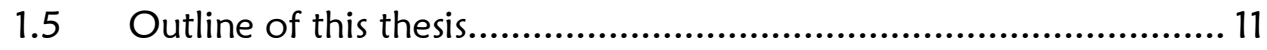

2. Slow-light propagation in a grated-waveguide cavity ..........................13

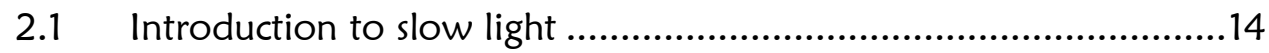

2.2 Slow light propagation in a grated waveguide cavity ....................15

2.2.1 Grated waveguide structure .................................................15

2.2.2 Theoretical methods for gratings ............................................16

2.2.3 Fabry-Perot resonance in a grated waveguide cavity .................18

2.3 Sensing applications and sensitivity analysis ................................20

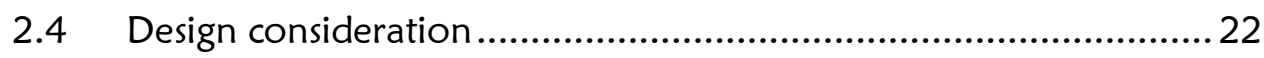

2.4.1 Ridge waveguide and grating parameters .............................. 22

2.4.2 Mechanical structure parameters ............................................ 24

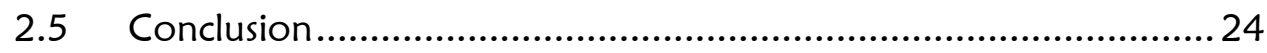


3. Bulk-index concentration and direct, label-free protein sensing .25

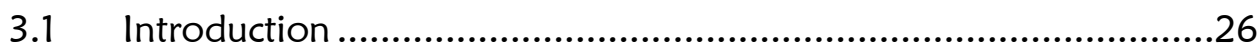

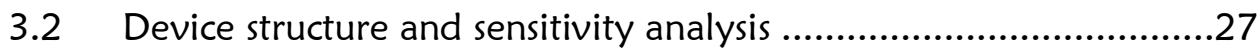

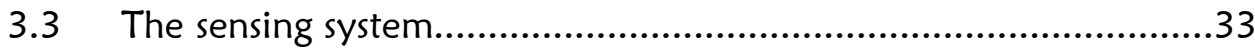

3.4 Bulk concentration sensing: results and discussion ........................35

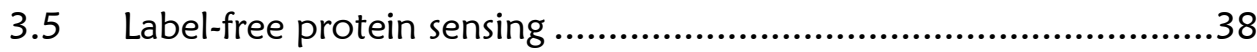

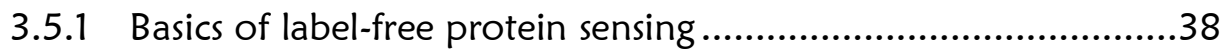

3.5.2 Basics of antigen-antibody interaction.......................................39

3.5.3 Immobilization of antibody on the $\mathrm{Si}_{3} \mathrm{~N}_{4}$ surface...................... 40

3.5.4 PepN enzyme sensing: results and discussion............................ 41

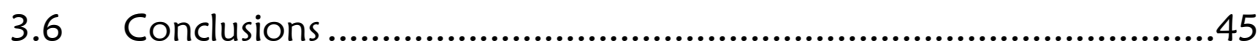

4. Mechano-optical read-out system for hydrogen sensor ........................47

4.1 Introduction and outline...........................................................48

4.2 Device structure and sensitivity ................................................48

4.3 Gas absorption induced cantilever bending................................. 50

4.4 Cantilever design consideration ................................................. 51

4.4.1 Residual stress-induced initial bending..................................... 51

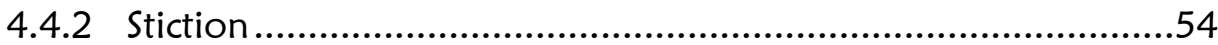

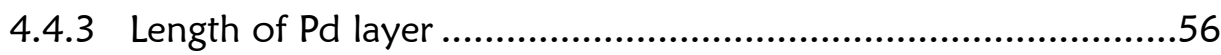

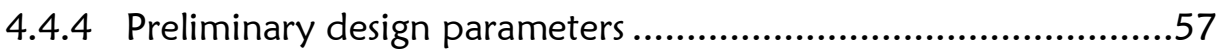

4.5 Fabrication and characterization of the mechano-optical read-out 57

4.5.1 Fabrication flow chart ..........................................................58

4.5.2 Fabrication issues and device characterization ..........................59

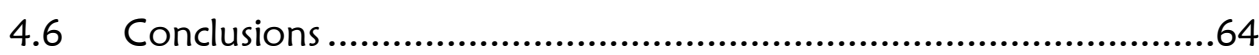

5. Mechano-optical hydrogen sensor: proof of concept ........................65

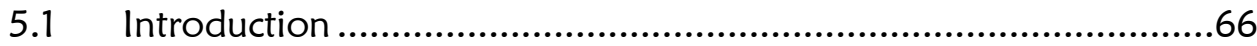

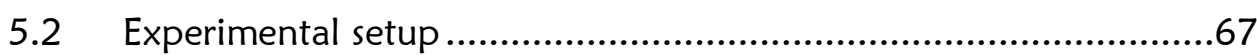

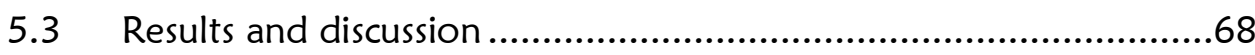


5.4 Structures for a sensor operating at high sensitivity ...................... 73

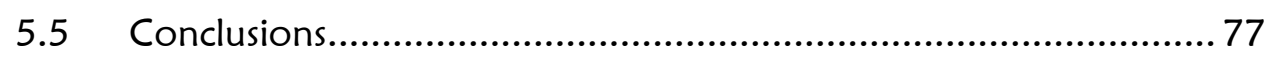

6. Mechano-optical hydrogen sensor: analysis and optimization..............79

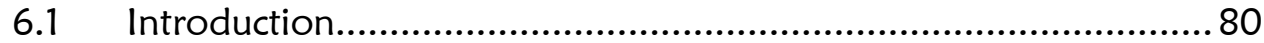

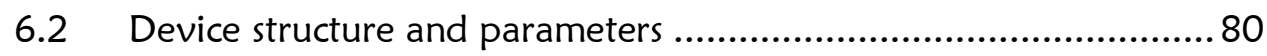

6.3 Device analysis and sensitivity ................................................. 82

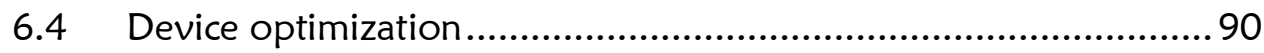

6.4.1 Bare grated waveguide ......................................................91

6.4.2 Integrated grated waveguide with cantilever........................... 95

6.4.3 Summary and conclusions on device optimization...................104

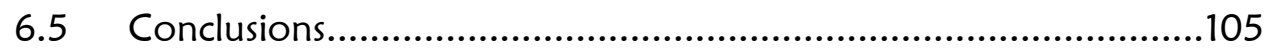

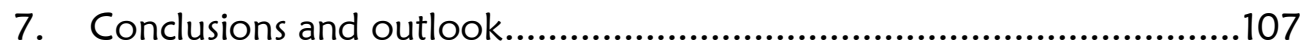

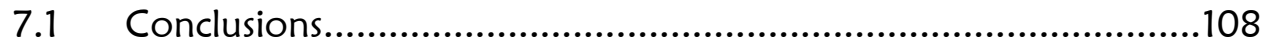

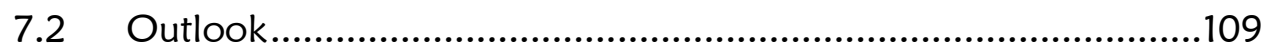

7.2.1 Improvement of device....................................................109

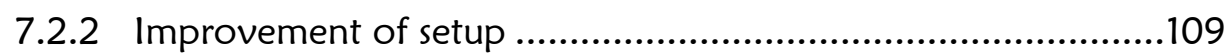

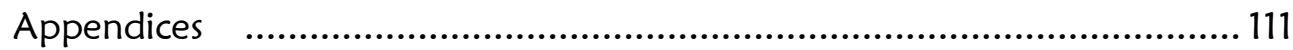

A1. Process of immobilizing antibodies on $\mathrm{Si}_{3} \mathrm{~N}_{4}$ surface ....................112

A2. Freeze-drying process...............................................................114

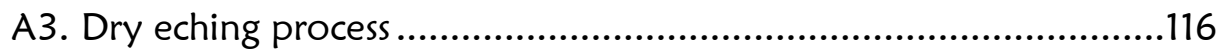

A4. Technology steps for the fabrication of the GWG-CL device.........117

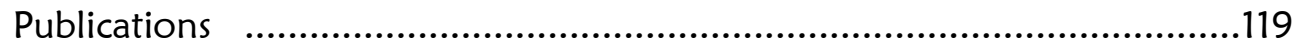

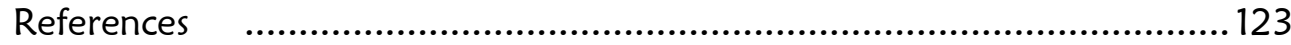

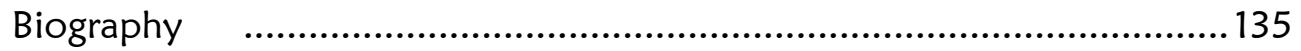




\section{List of Abbreviations}

$\begin{array}{ll}\text { Ab } & \text { Antibody } \\ \text { AFM } & \text { Atomic force microscopy } \\ \text { Ag } & \text { Antigen } \\ \text { BEP } & \text { Bidirectional eigenmode propagation } \\ \text { BC } & \text { Bare grating } \\ \text { CFE } & \text { Cell-free extract } \\ \text { CL } & \text { Cantilever } \\ \text { dCL } & \text { Doubly-clamped cantilever or bridge } \\ \text { FDTD } & \text { Finite-difference time-domain } \\ \text { FEM } & \text { Finite element method } \\ \text { GMS } & \text { Grating mode solver } \\ \text { CWC } & \text { Grated waveguide } \\ \text { GWCC } & \text { Grated waveguide cavity } \\ \text { IO } & \text { Integrated Optical } \\ \text { LIL } & \text { Laser interference lithography } \\ \text { LOD } & \text { Limit of detection } \\ \text { LPCVD } & \text { Low-pressure chemical vapor deposition } \\ \text { PBS } & \text { Phosphate buffered saline } \\ \text { PECVD } & \text { Plasma-enhanced chemical vapor deposition } \\ \text { RIU } & \text { Refractive index unit } \\ \text { SCL } & \text { Singly-clamped cantilever } \\ \text { SEM } & \text { Scanning electron microscope } \\ \text { TEOS } & \text { Tetraethyl orthosilicate } \\ \text { TMAH } & \text { Tetramethylammonium hydroxide } \\ \text { TMM } & \text { Transfer matrix method } \\ \text { WC } & \text { Waveguide } \\ & \end{array}$




\section{List of Figures}

Fig. 1.1 Detection principle of sensors: an example of specific detection........2

Fig. 1.2 Optical resonant cavities: (a) microsphere [27-29], (b) microtoroid [30, 31], (c) microring [16, 21, 32, 34], (d) 2D photonic crystal cavity [35, 36], (e) 1D photonic crystal cavity [37], and (f) grated waveguide (this work, Chapter 3).................................................................. 4

Fig. 1.3 A typical response curve (reproduced from [43]) for a cantilever covered with a Pd layer, with a thickness of $50 \mathrm{~nm}$. 8

Fig. 1.4 Schematic drawings of $\mathrm{Si}_{3} \mathrm{~N}_{4}$ grated-waveguide configurations for sensing applications: (1) Bulk homogeneous concentration sensing, (2) label-free protein sensing (surface sensing) and (3) mechano-optical gas sensing.

Fig. 2.1 Schematic of a grated waveguide (GWG) with the grated section or grating etched in a shallow ridge waveguide: (a) 3D schematic, and (b) $2 \mathrm{D}$ cross-section of the ridge (and teeth of the grating) and (c) 2D crosssection of the grating. 16

Fig. 2.2 Schematic of light behavior in a GWG. 17

Fig. 2.4 (a) Calculated transmission spectrum of a grating with a length of 125 periods; the highlighted resonant peak $A$ is used for sensing. (b) Zoomed part of the lower branch of the dispersion curve in Fig. 2.3 indicating also the $1^{\text {st }}$ resonances $(A, B$ and $C$ ) near the band edge for CWGC lengths of $L_{A}=125 \Lambda, L_{B}=250 \Lambda$ and $L_{C}=375 \Lambda$ and grating depth of $55 \mathrm{~nm}$ (other parameters given in Table 2.1). .19

Fig. 2.5 Schematic 2D cross-section of the considered GWG-based devices for (a) bulk, (b) surface, and (c) mechano-optical sensing. 20

Fig. 2.6 Effective index as a function of the $\mathrm{Si}_{3} \mathrm{~N}_{4}$ guiding layer thickness using a 1D mode solver for a three layer structure, single mode operation is obtained with thickness smaller than $620 \mathrm{~nm}$. 23

Fig. 3.1 A 3D schematic structure of the GWG device 27

Fig. 3.3 Schematic 2D cross-section of the device for bulk concentration sensing...... 29

Fig. 3.4 (a) Simulated and measured resonant wavelengths as a function of refractive index of top cladding. The dashed line displays calculated 
results assuming air-filled grating grooves. (b) Transmission spectra with air and water cladding (water-filled grooves) 36

Fig. 3.5 Transmission spectra of the concentration sensor, and (b) wavelength shifts and deduced concentrations due to changes of refractive index as a function of time.

Fig. 3.6 Antigen-antibody (Ag-Ab) interactions: an $\mathrm{Ag}$ binds strongly to its homologous $A b$ and vice versa by non-covalent interactions, based on intermolecular forces, i.e., electrostatic (ionic) forces, hydrogen bonding, hydrophobic bonding and Van der Waals forces (courtesy from Ref. [97]). 40

Fig. 3.8 Schematic 2D cross-section of the device for direct, label-free surface bio-sensing. 41

Fig. 3.9 Resonant wavelength of a CWC sensor after the different treatments: (0) as fabricated, (1) cleaning and surface activation - air top cladding; (2) silanization, (3) glutaraldehyde treatment, (4) antibody immobilization, (5) Blocking of remaining aldehyde groups - watery top cladding. The blue-shift from step 0 to step 1 is due to the thickness of $\mathrm{Si}_{3} \mathrm{~N}_{4}$ reduced after cleaning process, while red-shifts after each step of immobilization process are due to the increase in refractive index of the bulk or adlayer growth. The big jump from (1) to (2) is caused by applying a watery top cladding. 42

Fig. 4.1 Schematic of the GWG-CL set-up 49

Fig. 4.2 Behavior of a bimaterial cantilever after release. Depending on the status of residual stress in each material and the difference between two ratios $\sigma_{01} / E_{1}$ and $\sigma_{02} / E_{2}$, the bilayer cantilever may bend upwards or downwards after release. Initial bending of the bilayer $\mathrm{CL}$ presented here $\left(\mathrm{Pd} / \mathrm{SiO}_{2}\right)$ is similar to the case (a), while its absorption induced bending is similar to the case $(f)$. 53

Fig. 4.3 Critical length of the cantilever as function of its thickness at different gaps, assuming an adhesion energy of water $\gamma_{s}=100 \mathrm{~mJ} / \mathrm{m}^{2}$ and Young's modulus $E_{2}=61 \mathrm{GPa}$. 55

Fig. 4.4 (a) Schematic 2D cross-section of the $\mathrm{CWG}-\mathrm{CL}$ device; the layer materials are identified in Fig. 4.1. (b) Optical loss as a function of the length of the Pd layer for different $\mathrm{SiO}_{2}$ thicknesses, simulated using a 
mode solver. To avoid unwanted absorption or scattering loss the cantilever is not covered in the region that overlaps the modal field. ..56

Fig. 4.5 Process flow chart of fabrication of an integrated mechano-optical sensing device. 58

Fig. 4.6. Atomic force microscopy (AFM) topographic images of $\mathrm{Si}_{3} \mathrm{~N}_{4}$ surface roughness, after (a) $S F_{6}$ dry-etching $\left(R_{q}=25.6 n m\right)$ and (b) TMAH wet etching $\left(R_{\mathrm{q}}=0.46 \mathrm{~nm}\right)$.

Fig. 4.7 High-resolution scanning electron microscopy (HR-SEM) image of a fabricated chip (a), grating (zoomed inset) with period of $490 \mathrm{~nm}$, fabricated by laser interference lithography (LIL), and suspended cantilever released by TMAH wet etching solution. Reduction of initial bending was obtained by $\mathrm{O}_{2}$ plasma treatment (b).

Fig. 4.9 Optical microscopic images of facets: (left) a rough and cracked cleaved facet and (right) a smooth facet obtained by the new technique.

Fig. 4.10 Fabricated chips with $\mathrm{O}_{2}$ plasma treatment (A, B, C) and their measured transmission spectra (D) of a 250-period grating, with an 800 $\mathrm{nm}$ thick cantilever suspended above it. Gaps between the GWC and the cantilever in A, B, C are different, owing to different metal pad lengths leading to differences in stress and, so, in initial bending.

Fig. 5.2 Initial bending of the bridge due to differences in residual stress of the films: (a) numerical simulation using INTELLISUITE software package, (b) experimental result attained by a white-light interferometer, showing an initial up-bending of $\sim 500 \mathrm{~nm}$ at the center of the micro-bridge.....71

Fig. 5.3 (a) Transmission curves of the device in response to the absorption (filtered and unfiltered curves) and (b) the amount of wavelength shift $\Delta \lambda p$ versus the reaction time: absorption (left-hand side) and desorption (right-hand side)

Fig. 5.4 Temperature dependence of the integrated optical read-out; the change of resonant wavelength shift is $16 \mathrm{pm} / \mathrm{K}$.

Fig. 5.5 Simulated down-ward initial bending of the two proposed tri-layer sCLs with structural parameters given in Table 5.4............................. 75

Fig. 5.6 Schematic 2D cross-section of a tri-layer CL, initial gap $g_{o} \approx 200 \mathrm{~nm}$ can be obtained by controlling the thicknesses of the PECVD and TEOS 
$\mathrm{SiO}_{2}$ layers in such a way that (a) $\delta_{o} \approx 0\left(t_{S i}=200 \mathrm{~nm}\right)$ or (b)

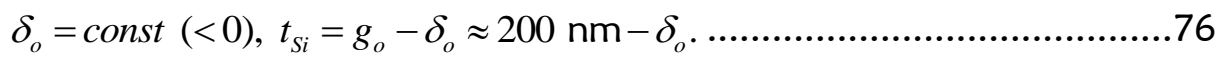

Fig. 6.1 The 3D schematic structure (a) and two cross-sections (b,c) of the GWG-cantilever device, and the simulated spectral shift due to varying the GWG-cantilever gap (d).... 81

Fig. 6.2 Dispersion curves of an infinitely long GWG (solid blue line) and the same GWG completely covered by a cantilever in close proximity ( $g=200 \mathrm{~nm}$ ) (dashed brown line) for two values of the grating depth $\mathrm{d}$ : (a) $\mathrm{d}=55 \mathrm{~nm}$, wavelength at the band edge of the grating is $\lambda_{\text {edge }}=1514.12 \mathrm{~nm}$, and (b) $\mathrm{d}=75 \mathrm{~nm}, \lambda_{\text {edge }}=1497.05 \mathrm{~nm}$. For both cases (a) and (b), the resonant state of a finite-length GWG of $L=125$ periods is indicated by a red dot, with $\lambda_{p}=1511.35 \mathrm{~nm}$ and $1495.10 \mathrm{~nm}$ for $\mathrm{d}=55$ $\mathrm{nm}$ and $75 \mathrm{~nm}$, respectively. 84

Fig. 6.3 (a) Schematic drawing of the relevant part of the band structure of a bare GWG and a GWG-cantilever system illustrating the two regimes discussed in the text. The red dots indicate the propagation constant(s) at resonant wavelengths for different values of $g$. (b) Schematic picture showing among others the nature of the modes for the two regimes. ..86

Fig. 6.4 (a) The quantity $\left|\partial \lambda_{p} / \partial g\right|$ as a function of g; the quantity is almost independent of the grating depth; and (b) Band-edge wavelength of the grating with and without a suspended cantilever (left-axis), and the (linear curve) $\ln \left[(-2 \gamma / B) \times\left(\lambda_{e, C L}-\lambda_{e, B G}\right)\right]$ (right-axis) for the two indicated values of the groove depth, confirming the exponential relationship presented in Eq. 6.4 (see text); 88

Fig. 6.5 Effect of groove depth, d, on GWG performance: (a) resonant wavelengths, and (b) transmittance at $\lambda_{p}\left(T_{p}\right)$ and maximum slope $\left(S_{\lambda, \max }\right)$ as functions of GWG groove depth. 92

Fig. 6.6 Effect of grating length $L$ on GWG performance: (a) resonant wavelengths as functions of the grating length, (b) difference between band edge wavelength and peak position as a function of $1 / L^{2}$ and (c) transmittance and maximum slope $S_{\lambda, \max }$ as a function of the grating length. Increasing the grating length results in a red-shift of the resonance peak towards to band-edge wavelength and an increase of $S_{\lambda, \max }$ (sensitivity) and loss 
Fig. 6.7 Computational results to find an optimum value for the cantilever thickness: (a) Resonant peak position, and (b) and (c) transmittance (leftaxis) and maximum slope $S_{\lambda \text {,max }}$ (right-axis) are plotted as functions of cantilever thickness $t(t=0$ means that the device is a grating without a suspended cantilever). 96

Fig. 6.10 Device performance at varying $W / L$. Transmittance at $\lambda_{p}\left(T_{p}\right.$ - leftaxis) and maximum value of $S_{\lambda}\left(S_{\lambda, \max }\right.$ - right-axis) are plotted as functions of the ratio $\mathrm{W} / \mathrm{L}$ (cantilever width/ grating length) of devices at lengths (a) $L_{1}=125 \Lambda$, (b) $L_{2}=250 \Lambda$ and (c) $L_{3}=375 \Lambda \ldots \ldots \ldots \ldots \ldots \ldots . . . . . . . . . . .101$

Fig. 6.11 Maximum value of $S_{\lambda}$ versus the grating length retrieved from Fig. 6.10 at $W / L=0.5$. .102

Fig. 6.12 Transmittance $T_{p}$ and $S_{\lambda, \max }$ as a function of the gap for parameters indicated in the figures. 103 


\section{List of Tables}

Table 1.1 Optical resonant cavities used for biosensors. ...............................4 Table 1.2 The quantity RI sensitivity ( $\mathrm{nm} / \mathrm{RIU}$ ) has often been used as a FOM for biosensors, however this is not a widely accepted FOM for the performance of various sensor configurations. ......................................

Table 1.3 Read-out methods used for cantilever-based hydrogen gas sensors. 9

Table 2.1 Fabrication parameters of the GWG structure shown in Fig.2.1. ...24

Table 4.1 Design parameters that need to be optimized to obtain a high $S_{C L}$, the blue boxed parameters can firstly be chosen, while the yellow boxed parameters require further consideration as presented in section 4.4. The co-ordinate system is given in Fig. 4.1................................................. 51

Table 4.2 Preliminary design parameters used for fabrication, which are not optimized yet.....................................................................................57

Table 4.3 Comparison of selectivity between $\mathrm{Si}$ and $\mathrm{SiO}_{2}$ and $\mathrm{Si}_{3} \mathrm{~N}_{4}$ using different etchants to remove poly-Si sacrificial layer (data from Ref. [131]) 60

Table 5.1 Notations used in this chapter. .67

Table 5.2 Dimensions and material properties of the CLs [126, 144] .68

Table 5.3. Initial bending $\delta_{0}$ of the $\mathrm{CLs}$ and the final fabricated gap

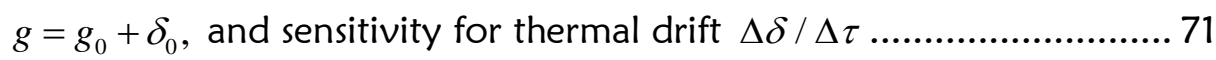

Table 6.1 Structural parameters of GWG-cantilever integrated system. ....... 80

Table 6.2 Overview of input and output parameters. Blue boxed values are chosen, yellow boxed values are varied to find an optimum design..... 91

Table 6.3 Overview of input and output parameters. Blue boxed values are chosen, yellow boxed values are varied to find an optimum design.....93

Table 6.4 Overview of input and output parameters. Blue boxed values are chosen, yellow boxed values are varied to find an optimum design.....95

Table 6.5 Overview of input and output parameters. Blue boxed values are chosen, yellow boxed values are varied to find an optimum design.....97

Table 6.7 Used parameters for Fig. 6.12. Blue boxed values are fixed, yellow boxed values are varied..................................................................... 102 


\section{Preface}

This dissertation is written as a partial fulfillment of the requirements to obtain the PhD degree at the University of Twente (UT). The PhD project was carried out at the Integrated Optical MicroSystems (IOMS) Group, MESA+ Institute for Nanotechnology, Faculty of Electrical Engineering, Mathematics and Computer Science (EEMCS) at UT in the period from the $1^{\text {st }}$ of September 2007 to the $31^{\text {st }}$ of August 2011.

The PhD project was financially supported by MEMSland, a project of the Point One program funded by the Dutch Ministry of Economic Affairs and the Dutch Technology Foundation - STW through project TOE 6596. The promoters for the project were:

Professor Markus Pollnau, IOMS - UT

Promoter

\section{Associate Professor Hugo J. W. M. Hoekstra, IOMS - UT}

Assistant promoter

A four-year PhD journey with wonderful experiences has reached its destination, and I owe my deep gratitude to many people for making this possible.

* First and foremost, my promoter, professor Markus Pollnau, and my daily supervisor and assistant-promoter, Dr. Hugo J. W. M. Hoekstra for giving me the opportunity to join the IOMS as a PhD student and for their kind and supportive guidance during the work, and for their patience in reading my paper manuscripts and thesis, and providing valuable feedback.

* My graduation committee: Prof. Dr. Ir. G. J. M. Krijnen, Prof. Dr. S. G. Lemay, Prof. Dr. Ir. D. Van Thourhout, Dr. Ir. R. M. de Ridder, for their time and efforts to review my thesis.

* Meindert Dijkstra and Dr. Lasse J. Kauppinen for their experimental support, especially in the first 2 years as my project partners and for their fruitful discussions during four years.

* Dr. Cuong Cao, Dr. Edwin T. Carlen, and Bach Le for their fruitful discussions about bio-sensing experiments.

* Prof. Dr. Paul Lambeck and Prof. Dr. Johan F. J. Engbersen for reviewing results of bio-sensing presented in Chapter 3. 
* Imran Akca and Nur Ismail for having been my office mates and sharing many things not only in research but also in daily life.

* Anton Hollink, Henk van Wolferen, and all the MESA+ cleanroom staff members for their technical support.

* Dr. Shanmugam Aravazhi (Abu), Marcel Hoekman, and Gabriel Sengo for sharing their experience in work, in life and especially for their insights on the spiritual journey and much more.

* IOMS colleagues: Laura Agazzi, Edward Bernhardi, Jonathan Bradley, Lantian Chang, Fehmi Civitci, Marko van Dalfsen, Chaitanya Dongre, Dimitri Geskus, Saara-Maarit Reijn, Fei Sun, Mustafa Sefünç, Sergio Vázquez-Córdova, Henri Uranus, and Ying Yang for making enjoyable and harmonic working environment.

* IOMS staffs: Prof. Dr. Alfred Driessen, Dr. Sonia García Blanco, Dr. Manfred Hammer, and Dr. Kerstin Wörhoff for their fruitful discussions and their motivating presence.

* IOMS secretaries Rita ter Weele-Stokkers, Annitta David, and Brigit Binkhorst-Reinshagen for their patience and administrative support.

* All Vietnamese friends at the UT for their unconditional support.

* All international friends in Enschede, in the Netherlands and in Europe for warming up my heart with their friendliness.

* All my Dhamma friends all over the world for their peaceful and harmonic company during the Vipassana meditation courses.

* Thao Dang, my best friend in high school, his family and friends, Tung Do and Karl Anderson, for having accompanied with me during my US trip in May 2011.

* And last but not least, all my family and friends, especially my parents for their unconditional love and caring since I was born. This thesis is dedicated to them. 


\section{Abstract}

\section{Integrated Optical Sensors utilizing Slow-light Propagation in Grated-waveguide Cavities}

Owing to the small size of integrated optical (IO) devices many basic functions can be integrated on one single 10 chip. 10 sensors are suitable candidates for accurate detection of small changes of physical or chemical parameters. The integration offers advantages such as enabling a high density of functionalities, automatic and stable alignment of elements, a high potential for mass production with in principle low production costs, and the possibility for the realization of sensor arrays for multi-parameter detection. The main goal of this PhD project is firstly to design, fabricate and demonstrate functioning $\mathrm{IO}$ devices based on grated waveguides for sensing applications.

A grated waveguide (GWG) is a waveguide with a finite-length grated section, being a structure with a periodic variation of the dielectric constant. Such a structure acts as both a 1-dimensional photonic crystal and, owing to modal reflections at the waveguide-GWG transitions, an optical resonator, as evidenced by fringes in the transmission spectrum. In particular near the band edge these fringes can be extremely sharp, which is related to both the near band edge shape of the dispersion curve, corresponding to slow light propagation, and high modal reflectance due to mode mismatch between waveguide and GWG modes. Both effects lead to strong light-matter interaction, which can be exploited for sensing applications. In this thesis, we demonstrate the versatility of a silicon nitride GWG optical cavity as a compact 10 sensor for bulk-index concentration sensing, label-free protein sensing and mechano-optical gas sensing.

For concentration sensing, the sensing principle is based on the bulk index change of the GWG top cladding. The principle of the label-free protein sensing relies on the growth and measurement of an adlayer on the GWC surface, owing to the antigen-antibody interaction. The mechano-optical gas sensing is based on stress-induced deflections of a cantilever suspended above the GWG, which are due to $\mathrm{H}_{2}$ gas absorption by the palladium receptor layer coated on the cantilever surface. 
In the first chapter of this thesis an overview is given of bio- and gassensors. In chapter 2, the background of slow light propagation in GWGs and its utilization for sensing applications are discussed. In chapter 3 , results related to the first 2 sensing applications (concentration sensing and label-free protein sensing) are presented; here, sensitivity and limit of detection of the sensors are analyzed in detail. The design and fabrication of the GWGcantilever integrated read-out, and the demonstration of the integrated mechano-optical sensor for gas sensing, are presented in chapter 4 and chapter 5, respectively. Results of an optimization study of the integrated mechano-optical read-out principle, on the basis of numerical calculations, is presented in chapter 6 . In chapter 7, conclusions and outlook, based on the results presented in this thesis, are given. 


\section{Samenvatting}

\section{Geïntegreerde optische sensoren, gebaseerd op vertraagde voortplanting van licht in golfgeleiders met een ruimtelijk-periodieke verstoring}

Vanwege de kleine afmetingen van geïntegreerde optische (IO) componenten kunnen in principe vele basisfuncties worden geïntegreerd op één enkele optische chip. 10 sensoren zijn geschikte kandidaten voor een nauwkeurige detectie van kleine veranderingen in fysische en chemische parameters. Integratie biedt voordelen zoals de mogelijkheid om een grote dichtheid van functionaliteiten te verkrijgen, automatische en stabiele uitlijning van elementen, de mogelijkheid voor massaproductie bij lage kosten en de realisatie van rijen sensoren om meerdere parameters gelijktijdig te detecteren. Het hoofddoel van dit project is voornamelijk om IO systemen, gebaseerd op tralie-golfgeleiders, te ontwerpen, te fabriceren en de toepasbaarheid voor sensorapplicaties te demonstreren.

Een tralie golfgeleider (TCG) is een golfgeleider met een traliesectie van een eindige lengte, waarin de diëlectrische constante periodiek varieert. Zo'n structuur functioneert als zowel een 1-dimensionaal fotonisch kristal als een optische resonator ten gevolge van de reflectie van modi op de overgangen van golfgeleider naar TGG, zoals blijkt uit de oscillaties in de transmissie. Transmissiepieken kunnen vooral vlakbij de bandkant erg scherp zijn, hetgeen verband houdt met zowel de vorm van de dispersiekromme en de daarmee corresponderende traagheid van het licht vlakbij de bandkant als de grote modale reflectie ten gevolge van modale veldverschillen tussen TCG en golfgeleider modi. Beide effecten leiden tot een sterke licht-materie wisselwerking, hetgeen gunstig is voor de gevoeligheid van sensoren. In dit proefschrift word de veelzijdigheid aangetoond van een dergelijke TCGtrilholte, met toepassingen als een compacte, 10 sensor voor concentratiedetectie op basis van de brekingsindex van een oplossing, voor label-vrije proteïnedetectie en voor mechano-optische gasdetectie.

Het detectieprincipe voor concentratiedetectie is gebaseerd op verandering van de brekingsindex van de te onderzoeken vloeistof die in contact is met de TGG. Het detectie principe voor label-vrije proteïne 
detectie is gebaseerd op de aangroei van een additionele laag als gevolg van antigen-antilichaam-reacties op het TGG oppervlak. Het detectieprincipe voor mechano-optische gasdetectie maakt gebruik van de buiging van een geïntegreerde, boven de TGG hangende, microbalk. De buiging wordt veroorzaakt door een mechanische spanning tengevolge van de door absorptie van $\mathrm{H}_{2}$ gas aan de palladium receptorlaag die op de microbalk is aangebracht.

Het eerste hoofdstuk van dit proefschrift geeft een overzicht van bio- en gas-sensoren. In hoofdstuk 2 worden de fysische fenomenen in de TGC verklaard en het gebruik daarvan voor sensortoepassingen besproken. In hoofdstuk 3 worden de eerste twee sensortoepassingen (concentratiedetectie en label-vrije proteïnedetectie) gepresenteerd. Hierbij wordt een gedetailleerde analyse gegeven van de gevoeligheid en detectielimiet. Hoofdstuk 4 behandelt het ontwerpen fabricage van de geïntegreerde detectie-eenheid die is opgebouwd uit TGG en microbalk. Mechano-optische gasdetectie wordt gedemonstreerd in hoofdstuk 5. Resultaten van een optimalisatiestudie, op basis van numerieke simulaties worden gepresenteerd in hoofdstuk 6. Hoofdstuk 7 geeft de conclusies alsmede een vooruitzicht op basis van de in dit proefschrift gepresenteerde resultaten. 


\section{Introduction}

The current state of the art in biosensors and gas sensors is summarized. Closer to the topics of this thesis: an overview of biosensors based on optical resonant cavities and an overview of cantilever-based gas sensors are presented and discussed. The chapter then addresses the novelty and the aim of this PhD project, followed by the outline of the thesis. 


\subsection{What is a sensor?}

A sensor is a device that can recognize the presence of a specific stimulus and translate it into a measurable signal [1]. A sensor consists of a receptor or recognition element recognizing the analyte (or target or measurand) and a signal transducer. The recognition element can be either associated with or integrated within the transducer. The sensor design focuses on specificity such that it reacts preferentially on a single stimulus.

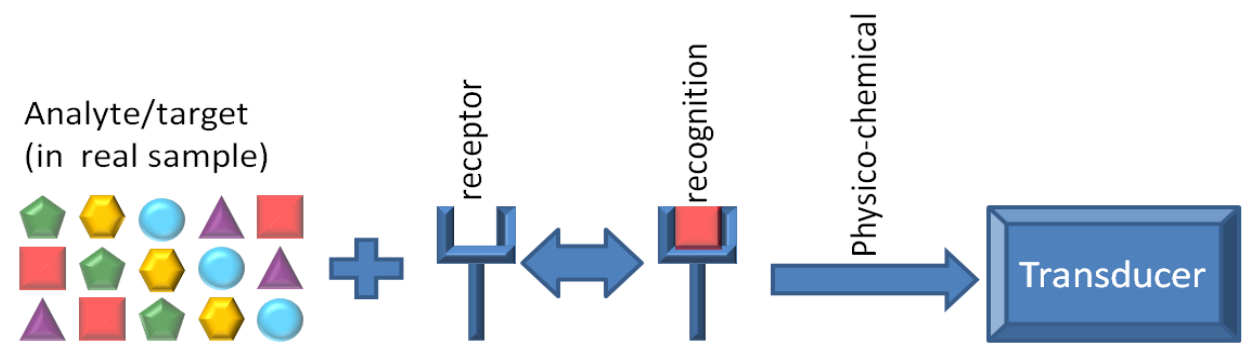

Fig. 1.1 Detection principle of sensors: an example of specific detection

\subsection{Biosensors}

\subsubsection{Overview of biosensors}

A biosensor is used to qualitatively and/or quantitatively detect biological molecules. Biosensors are widely used in quality assurance in agriculture, food and pharmaceutical industries, monitoring environmental pollutants and biological warfare agents, medical diagnostics, biological assays [2-4]. Depending on type of bio-recognition molecule, there are two categories of biosensors: 1) catalytic biosensors, of which the recognition molecule such as enzymes or microorganisms, catalyzes a reaction involving the analyte to give a product monitored by means of electrochemical, photometric, thermometric or acoustic detection systems [5], and 2) affinity biosensors, which are characterized by a binding event between the recognition molecule and the analyte. Transduction of the biorecognition event then becomes challenging and has been achieved using labeled species or unlabeled (label-free) targets approaches [3, 6, 7].

In the labeled detection approach, labeled species are attached to the target, and detected using an optical or electrochemical transducer depending on whether the labeled species are optically active or electroactive. The amount of bound targets is inferred from the amount of 
labeled species being detected. However, labeling a biomolecule can change its binding properties and thus the yield of the target-label coupling reaction is highly variable $[2,6]$.

In the label-free detection approach, the target analyte is not labeled but bound specifically to the corresponding receptor immobilized on the transducer surface. Any change on this surface can be monitored using electrical (e.g., current, voltage, impedance, piezoelectric) [8-11], mechanical (e.g., quartz crystal microbalance or resonant cantilever) [12-15] or optical read-out methods $[16,17]$.

\subsubsection{Label-free biosensors based on optical resonant cavities}

As mentioned above, label-free detection typically is performed using an electrical, mechanical or optical transducer. All of these transduction methods are sensitive, however, they are all require surface functionalization (i.e., antibody, antigen, $\mu \mathrm{RNA}$, etc) $[3,16-18]$ in order to detect a specific analyte. Optical biosensors operate by exploiting the interaction of the optical field with its environment, usually via the evanescent modal field [19, 20]. The sensing mechanism may take advantage of resonant-cavity-based detection or surface-plasmon-based detection [21-26]. As the research presented here focuses on optical grated-waveguide cavities, it is relevant to provide a brief summary of detection methods based on optical resonant cavities in general, for comparison.

Optical resonant cavities confine light at a certain wavelength range which is defined by the cavity. Molecules bound to the cavity surface will result in a shift of resonant frequency of the cavity. Therefore, bio-detection occurring when bound molecules interact with the evanescent field of modes supported by the the cavity can be performed by monitoring changes in the resonant frequency (wavelength) of the cavity. The sensitivity of resonant cavity based sensors depends on the figure of merit (FOM) of the cavity - the quality factor ( $Q$-factor). This term describes the photon lifetime of the cavity, which is directly related to the optical losses of the cavity. A device with a high $Q$ has low optical losses and a long photon lifetime, leading to strong photon-molecule interactions and therefore to high sensitivity [17].

Several resonant cavity geometries and their applications for label-free bio sensing have been reported in Refs. [16, 21, 27-37]. An overview of these cavities and the grated-waveguide based cavity presented in this thesis is contained in Fig.1.2 and Table 1.1. 

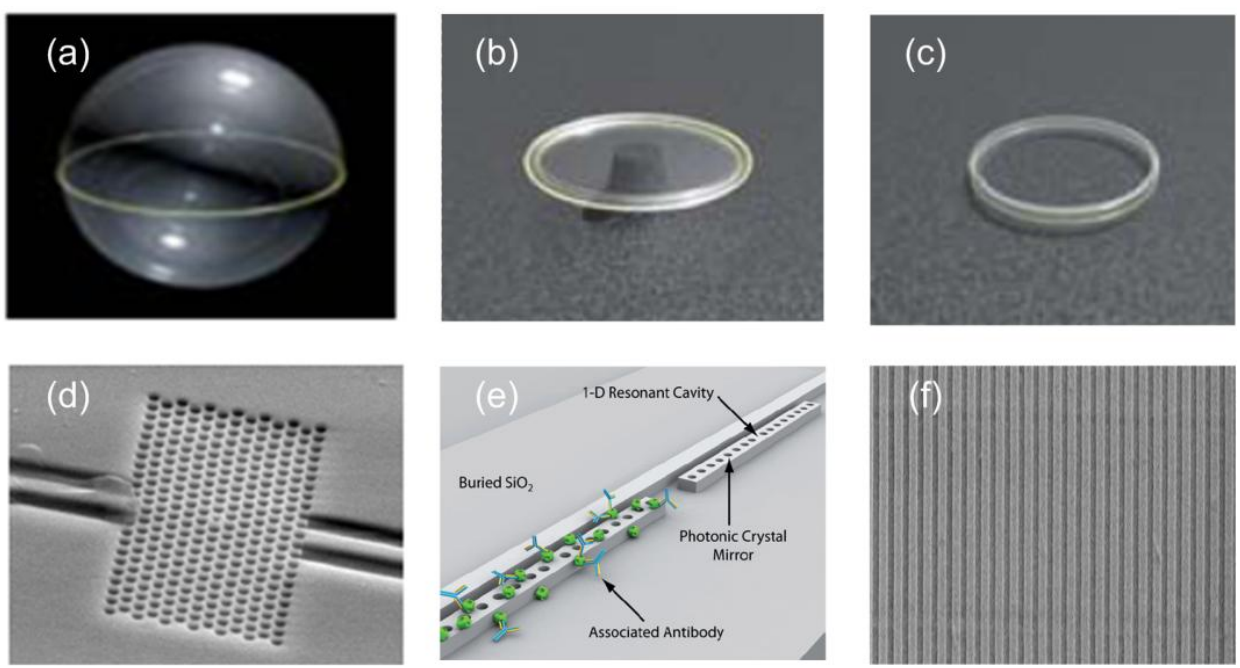

Fig. 1.2 Optical resonant cavities: (a) microsphere [27-29], (b) microtoroid [30, 31], (c) microring [16, 21, 32, 34], (d) 2D photonic crystal cavity [35, 36], (e) 1D photonic crystal cavity [37], and (f) grated waveguide (this work, Chapter 3).

Table 1.1 Optical resonant cavities used for biosensors.

\begin{tabular}{llrrl}
\hline Cavity & Material & $Q$ in air & $Q$ in water & Detection demonstration \\
\hline Microsphere & Silica & $>10^{9}$ & $>10^{6}$ & $\begin{array}{l}\text { Single virus, cis/trans of } \\
\text { protein, DNA }\end{array}$ \\
Microtoroid & Silica & $>10^{8}$ & $>10^{8}$ & $\begin{array}{l}\text { Single molecule, } \\
\text { fluorophore }\end{array}$ \\
Microring & $\begin{array}{l}\text { Polymers, } \\
\text { Silicon }\end{array}$ & $\sim 10^{3}-10^{5}$ & $\sim 10^{3}-10^{5}$ & Bacteria \\
Photonic & Silicon & $\sim 10^{2}-10^{4}$ & $\sim 10^{2}-10^{4}$ & Streptavidin \\
crystal cavities & & & & \\
$\begin{array}{l}\text { GWC cavity } \\
\text { (this work) }\end{array}$ & $\begin{array}{l}\text { Silicon } \\
\text { nitride }\end{array}$ & $\sim 10^{3}-10^{5}$ & $\sim 10^{3}-10^{5}$ & PepN enzyme (protein) \\
\hline
\end{tabular}


It is noted that a comparison between different sensor technologies and/or device configurations is impractical owing to absence of a widely accepted FOM. The quantity "RI sensitivity" defined as the resonant wavelength shift (nanometer) per unit refractive index change (nm/RIU nanometer per refractive index unit), which has often been used to quantify performance of surface-plasmon-based sensors, has also been quoted for others (see Table 1.2) $[16,25,26,33,38]$. However, this criterion fails in taking into account factors that can significantly affect the performance of resonant-cavity-based sensors. For instance, high- $Q$ resonator with narrow linewidth of resonant peak will largely affect the wavelength resolution.

Table 1.2 The quantity $R I$ sensitivity $(\mathrm{nm} / \mathrm{RIU}$ ) has often been used as a FOM for biosensors, however this is not a widely accepted FOM for the performance of various sensor configurations.

\begin{tabular}{lll}
\hline Device & RI sensitivity (nm/RIU) & Reference \\
\hline Surface plasmon-based sensors & $10^{3}-10^{4}$ & {$[25,26]$} \\
Slot-waveguide & 212 & {$[33]$} \\
Grated silicon photonic wire & 160 & {$[38]$} \\
Ring resonator & 163 & {$[16]$} \\
GWG (this work) & 140 & Chapter 3 \\
\hline
\end{tabular}

\subsection{Gas sensors}

Trace gas sensing in the ppb-ppt (parts per bilion-trillion) range is quite relevant in a large number of application fields such as health-care, food and feed processing, agriculture, forensics and safety. For these fields there is a strong need for easy to handle, cheap and small sized equipment for direct, in situ detection of a multitude of gas components simultaneously, with minor or no sample preparation and in an accurate, sensitive and reliable way [39].

Below we will review relevant aspects of the state of the art of methods for trace gas sensing, and cantilever-based gas sensors. 


\subsubsection{Trace gas sensing methods}

A large variety of different methods for trace gas sensing can be discerned. Of these the most well known ones will be discussed, which can roughly be subdivided in:

1. Spectroscopic methods, which include spectral absorption, photoacoustics, Fourier transform infra-red spectroscopy and differential absorption spectroscopy [39].

2. Physical methods like gas chromatography or mass spectroscopy.

3. Chemical methods such as chemiluminescence.

4. Methods utilizing conductivity changes in suitable receptor materials owing to adsorption or absorption of gasses [40, 41].

5. Methods using the change of optical properties in receptor materials [42].

6. Set-ups that detect cantilever deflection owing to stress due to sorption of trace gasses by a receptor layer on top of the cantilever $[43,44]$.

All the above methods have their merits, depending on the application field.

The spectral methods are in principle highly sensitive but require dedicated and usually relatively large equipment, including a tunable light source or a monochromator and generally long interaction lengths. Wide application of these methods is often hampered by the fact that no laser is available to scan over a suitable absorption line of a certain gas molecule. Among the spectral methods photo-acoustics is in particular sensitive and widely applied [45] owing to the fact that it is a so-called zero-method, whereby the signal is proportional to the concentration of the target gas.

Chemical and physical methods are in general highly sensitive; a wide application is hampered by the fact that tedious and target gas dependent sample preparation is required for the chemical methods and that generally the required equipment is large and expensive.

Set-ups, utilizing sorption of the target molecule at a receptor layer have a large potential for the realization of cheap and compact devices, for a large number of gasses. Quite relevant for the application of these devices is the availability of a large number of receptor materials, such as metal oxides, metals (like Pd) and polymers [46]; however, selectivity is for many of these materials an issue of concern. The resolution of sorption-based devices 
depends on the used read-out principle. Sensing on the basis of conductivity changes, mostly applied using metal oxides at an elevated temperature of a few hundreds degrees, enable compact and relatively simple set-ups, suitable to detect gas concentrations with a resolution mostly not better than in the ppm range [40].

To our knowledge there are not many publications on practical implementations of sensors utilizing the change in optical properties of gas absorbing materials [42, 47, 48]. Devices based on that principle have been reported to have a resolution of at best in the ppm region. An extra complication for such sensors is that light absorbing materials, like the metal $\mathrm{Pd}$, cannot be used in an effective way in such sensors.

Quite promising for trace gas sensing devices, meeting all the requirements mentioned above (at the beginning of this section) are devices based on the read-out of gas induced deflection of cantilevers. Depending on the used receptor-target combination, devices with a piezo-resistivity based read-out attain a resolution in the ppm-ppb region [49], corresponding to a typical detection limit for the cantilever deflection in the picometer (pm) region [50]. This level of resolution can also be attained by other, presently used read-out principles for the deflection of cantilevers mentioned in the next section.

\subsubsection{Cantilever-based gas sensors}

\section{* Coated micro-cantilevers based gas sensors}

Only since recently [51] cantilevers coated with molecule-specific receptor layers are being used for sensing purposes, in particular for bio-sensing [52]. Owing to their large sensing potential there is a rapid growth of the field as shown by the number of journal papers and start-up companies related to this topic. Micro cantilevers are mostly made of $\mathrm{Si}, \mathrm{SiO}_{2}, \mathrm{Si}_{3} \mathrm{~N}_{4}$ or polymeric materials like SU8. Typical dimensions are 10-500 $\mu \mathrm{m}$ in lateral directions and thicknesses mostly in the 10-100 nm range. Fabrication methods are based on patterning of a suitably chosen layered structure, followed by removal of a sacrificial layer underneath the cantilever [53]. In general, the fabrication methods for cantilevers are well established and arrays with some thousands of cantilevers are being fabricated on a single chip [52].

Changes due to molecular absorption by a receptor layer on top of a cantilever can be measured either in the dynamic range, whereby the change 
in cantilever mass is measured via the shift in resonance frequency [53], or in the static mode, in which stress-induced bending is detected [52]. Both read out principles have a large potential, the static mode is most often used (as in this thesis) for its relative simplicity. Reported cantilever read out methods are on the basis of changes in capacitance $[43,44,54]$, changes in the piezoresistivity $[49,50,55]$, and optical beam deflection detected with a position sensitive detector, see for example reference $[52,56]$. These methods have a typical deflection resolution of at best $\sim 1 \mathrm{pm}$.

\section{* Cantilever based hydrogen gas sensing}

A typical response curve of the (static) deflection of a cantilever covered with $\mathrm{Pd}$ to detect $\mathrm{H}_{2}$ and read out at ambient temperature via a change in capacitance is given in Fig. 1.3 (reproduced from [43]).

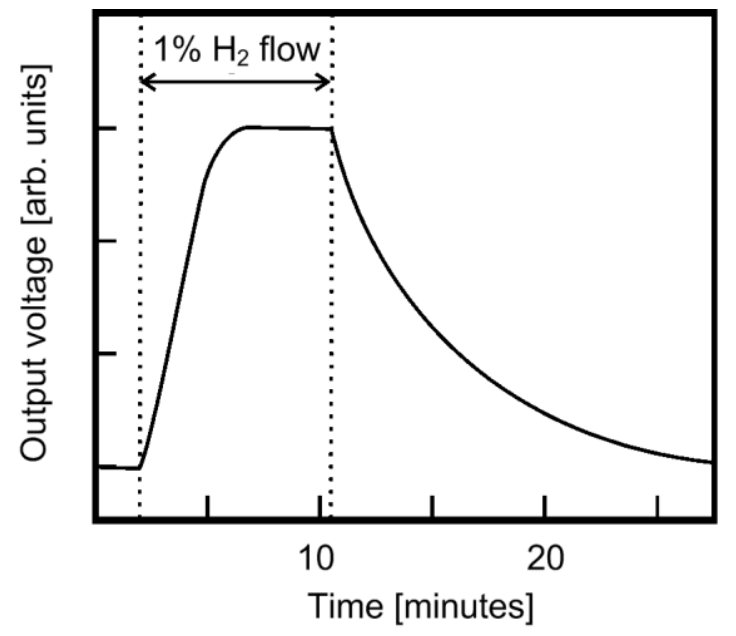

Fig. 1.3 A typical response curve (reproduced from [43]) for a cantilever covered with a $P d$ layer, with a thickness of $50 \mathrm{~nm}$.

It can be seen that for the used Pd thickness of $\sim 50 \mathrm{~nm}$ the response time is in the order of minutes. As shown by reference [43] the measuring time can be reduced considerably by measuring the slope of the response curve (i.e., the bending speed) from which the $\mathrm{H}_{2}$ concentration can also be obtained in an accurate way. It is anticipated that, if the latter is not constant and knowing the reaction kinetics of the $\mathrm{Pd}-\mathrm{H}_{2}$ combination, also the $\mathrm{H}_{2}$ concentration as a function of time can be determined. It is also relevant to note that the absorbed amount of hydrogen is proportional to the square root of the gas concentration. This is the so-called Sieverts' law $[43,57,58]$. 
This square root dependence is related to the fact that Pd absorbs hydrogen atoms, which have to recombine as molecules at the surface before entering a gas phase. As a consequence of the above, the response of a $\mathrm{Pd}$ covered cantilever is proportional to the square root of the $\mathrm{H}_{2}$ concentration, which increases the dynamic range of the system considerably. For example, reducing the $\mathrm{H}_{2}$ concentration to a value of $0.01 \%$ of the original value leads to a reduction of the response to a value of $1 \%$ of the original response.

Illustration of cantilever-based hydrogen gas sensors with different readout methods is contained in Table 1.3.

Table 1.3 Read-out methods used for cantilever-based hydrogen gas sensors.

\begin{tabular}{llll}
\hline Read-out method & Output signal & $\begin{array}{l}\mathrm{H}_{2} \text { concentration } \\
\text { in demonstration }\end{array}$ & Reference \\
\hline Optical beam deflection & Optical signal & $0.1 \%-4 \%$ & {$[56]$} \\
Capacitance & Voltage & $0.01 \%-0.1 \%$ & {$[43]$} \\
& & $0.4 \%-10 \%$ & {$[54]$} \\
Piezoresistance & Voltage & $2.5 \%$ & {$[55]$} \\
GWG (this work) & Optical & $1 \%$ & Chapter 5 \\
& transmission & & \\
\hline
\end{tabular}

\subsection{Novelty and aim of this thesis}

A grated waveguide cavity (GWCC), which is a waveguide with a finitelength grated section, acts as an optical resonator, exhibiting sharp fringes in the transmission spectrum near the stop-band edges of the grating. These oscillations are due to Fabry-Perot resonances of Bloch modes propagating in the cavity defined by the grated section [59, 60]. Any small structural perturbation in the environment of the GWG, which disturbs the evanescent field of the GWC resonant modes, will lead to a shift of its transmission spectrum. Such an effect can be exploited for sensing applications. In 2005, our IOMS group fabricated and demonstrated an optical GWCC as a readout for bulk-index evanescent field sensing with a resolution for index changes of $\Delta n \cong 4 \times 10^{-4}$ in the visible wavelength range [59]. As a follow-up, one aim of this $\mathrm{PhD}$ project is to fabricate and demonstrate the low loss 
GWGCs for bulk index concentration sensing (homogeneous sensing) with improved sensitivity. In addition, two other sensing applications of the GWGCs are exploited: (1) label-free protein sensing (surface sensing), where the GWGC spectral shift is due to the antigen-antibody interaction leading to the growth of an adlayer on top of the cavity, which is detected via the spectral shift of transmittance; and (2) mechano-optical (MO) gas sensing, based on the integration of a GWG and a cantilever (CL), where a palladium receptor layer is coated on the surface of a silicon oxide $\mathrm{CL}$ suspended above a GWG, which leads to surface-stress-induced bending due to absorption of $\mathrm{H}_{2}$ gas, which is also detected via the spectral shift of the transmittance. Owing to the MO interaction the evanescent field based sensing of gases comes within reach of 10 devices.

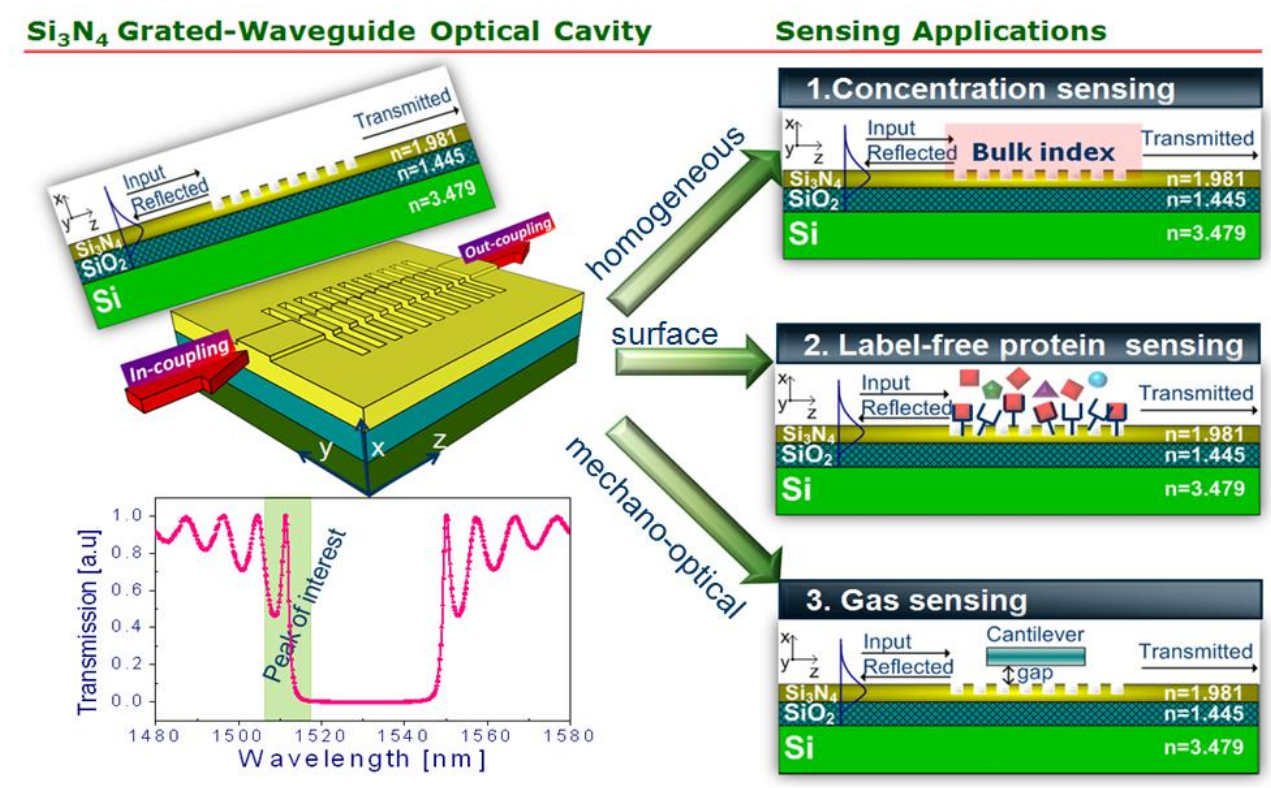

Fig. 1.4 Schematic drawings of $\mathrm{Si}_{3} \mathrm{~N}_{4}$ grated-waveguide configurations for sensing applications: (1) Bulk homogeneous concentration sensing, (2) labelfree protein sensing (surface sensing) and (3) mechano-optical gas sensing. 


\subsection{Outline of this thesis}

This thesis consists of 7 chapters:

Chapter 1 gives a general introduction to the research

Chapter 2 describes properties of a grated-waveguide cavity, the core element exploited for sensing applications presented in this thesis.

Chapter 3 presents two applications of the bare GWGs for bulk-index concentration, and label-free protein biosensing.

Chapter 4 describes the fabrication and a proof of concept of the GWG$\mathrm{CL}$ integrated device as a novel read-out platform for nanodisplacements

Chapter 5 illustrates characterization and proof of concept of the GWGcantilever integrated device as a compact mechano-optical sensor for hydrogen gas detection.

Chapter 6 describes the simulation and optimization of the GWG-CL mechano-optical read-out.

Finally, chapter 7 presents the general conclusions of the thesis and provides an outlook for possible developments of the GWG and GWG-CL based sensors.

The work of this $\mathrm{PhD}$ project has been producing 4 journal papers based on chapter 3 (S.V. Pham et al., submitted 2012), chapter 4 (S. V. Pham et al., 2011, Photonics Technology Letters), chapter 5 (S. V. Pham et al., 2011, Optics Letters), and chapter 6 (S. V. Pham et al., 2012, to be submitted). 



\section{Slow-light propagation in a grated- waveguide cavity}

In this chapter, slow-light propagation in a grated waveguide structure and its utilization for sensing applications is described as a background for latter chapters. Slow-light propagation in a grated waveguide is explained through its band structure (dispersion curve). Three sensing applications are proposed and their sensitivity is generally defined. The role of the slow-light effect for high sensitivity in the grated waveguide is clarified. Design considerations for the realization of grated waveguide based sensors are also discussed in this chapter. 


\subsection{Introduction to slow light}

The velocity of light in vacuum, $c$, is approximately $3 \times 10^{8} \mathrm{~m} \cdot \mathrm{s}^{-1}$. When a plane wave of light propagates through a dielectric material, its phase velocity along the propagation direction is lower than $c$. This reduction in speed is quantified by a factor called the refractive index of the material - $n$, which is the ratio between $c$ and the phase velocity of the plane wave. The group velocity corresponds to the speed of an optical pulse in a certain medium and one may speak of slow light if the optical pulse propagates at a low speed compared to $c$ [56-72]. The group velocity is defined as

$$
v_{g}=\frac{d \omega}{d k},
$$

where $k$ is the wavenumber and $\omega$ is the angular frequency. The group index $n_{g} \equiv c / v_{g}$, which is regarded as a slow-down factor, is given by

$$
n_{g}=\frac{c}{v_{g}}=c \frac{d k}{d \omega}=\frac{d(n \omega)}{d \omega}=n+\omega \frac{d n}{d \omega}=n-\lambda \frac{d n}{d \lambda},
$$

with $\lambda$ the wavelength.

It is obvious as shown in Eq. 2.2 that the group index $n_{g}$ depends not only on the refractive index of the material - $n$, but also on the way in which the refractive index changes with frequency, i.e., the derivative of refractive index with respect to frequency - $d n / d \omega$. The refractive index of material is neither very large nor easy to modify and takes typical values between $\sim 1.5$ and $\sim 3.5$ for glasses and semiconductors, respectively [61]. Thus, a slow light effect is not due to an abnormally large refractive index, but may occur in uniform materials if the group index $n_{g}$ is greatly enhanced with a large firstorder dispersion $d n / d \omega$. Accordingly, the group velocity $v_{g}\left(=c / n_{g}\right)$ can be greatly reduced by a large dispersion $(\omega d n / d \omega>>0)$, arising from an optical resonance within the material or structure. An example of a dispersive structure is a waveguide having a regular spatial perturbation along its length in which case the above expressions also hold with $n$ now referring to a modal index.

By utilizing dispersive materials, for instance, Hau et al. [62] observed light slow down to $17 \mathrm{~m} . \mathrm{s}^{-1}$ at an ultralow temperature in a Bose-Eistein condensate. Kash et al. [63] slowed light down to $90 \mathrm{~m} . \mathrm{s}^{-1}$ in rubidium 
vapor. This experiment was then refined by Budker et al. [64], and light was slowed down to $8 \mathrm{~m} \cdot \mathrm{s}^{-1}$. Finally, Bigelow et al. [65] were able to slow light down to less than $58 \mathrm{~m} \cdot \mathrm{s}^{-1}$ at room temperature in a ruby crystal. By utilizing dispersive structures for slowing down light, optical resonators such as photonic-wire waveguides $[66,67]$, photonic crystals $[68,69]$, ring resonators $[70,71]$ were demonstrated. These highly dispersive structures are more easily applicable and so more suitable than dispersive materials for the on-chip integration and room-temperature operation of slow-light devices.

In this thesis, the author considers slow-light that occurs at wavelengths near the photonic band edge of a grated waveguide (GWG) and exploits it for sensing applications. This chapter is organized as follows. Section 2.2 describes the GWG structure and its slow-light properties by considering its dispersion curve (band structure). In Section 2.3, sensing applications are presented and their sensitivity is defined. Design considerations of the devices are then discussed in Section 2.4. Finally, a conclusion is given in Section 2.5.

\subsection{Slow light propagation in a grated waveguide cavity}

\subsubsection{Grated waveguide structure}

A grated waveguide (CWG) or waveguide grating is a waveguide with a finite-length grated section and it is formed by a periodic variation in the dielectric constant along the propagation direction [60]. A three dimensional (3D) and a 2D cross-section schematic of such a GWG are depicted in Fig. 2.1. The GWG structure presented in this work, which is formed by a $\mathrm{Si}_{3} \mathrm{~N}_{4}$ guiding layer deposited onto a $\mathrm{SiO}_{2}$ substrate, with air as a superstrate, is divided into 3 blocks: the input waveguide (WG), the grated section (grating) and the output WG. The material indices of $\mathrm{SiO}_{2}$ and $\mathrm{Si}_{3} \mathrm{~N}_{4}$ at infrared wavelengths are approximately 1.445 and 1.981, respectively, and they are nearly constant (i.e., an index change $\sim 0.0001 \%$ per nanometer wavelength (or $\sim 10^{-6} \mathrm{RIU} / \mathrm{nm}$ )) in the wavelength range of $1.48-1.58 \mu \mathrm{m}$ ) [72]. Material dispersion of the material system $\left(\mathrm{SiO}_{2} / \mathrm{Si}_{3} \mathrm{~N}_{4}\right)$ thus has only a very small effect on the GWG performance and can be neglected. Therefore, slow-light propagation in the $\mathrm{Si}_{3} \mathrm{~N}_{4}$ GWG is mainly due to structurally induced dispersion. 

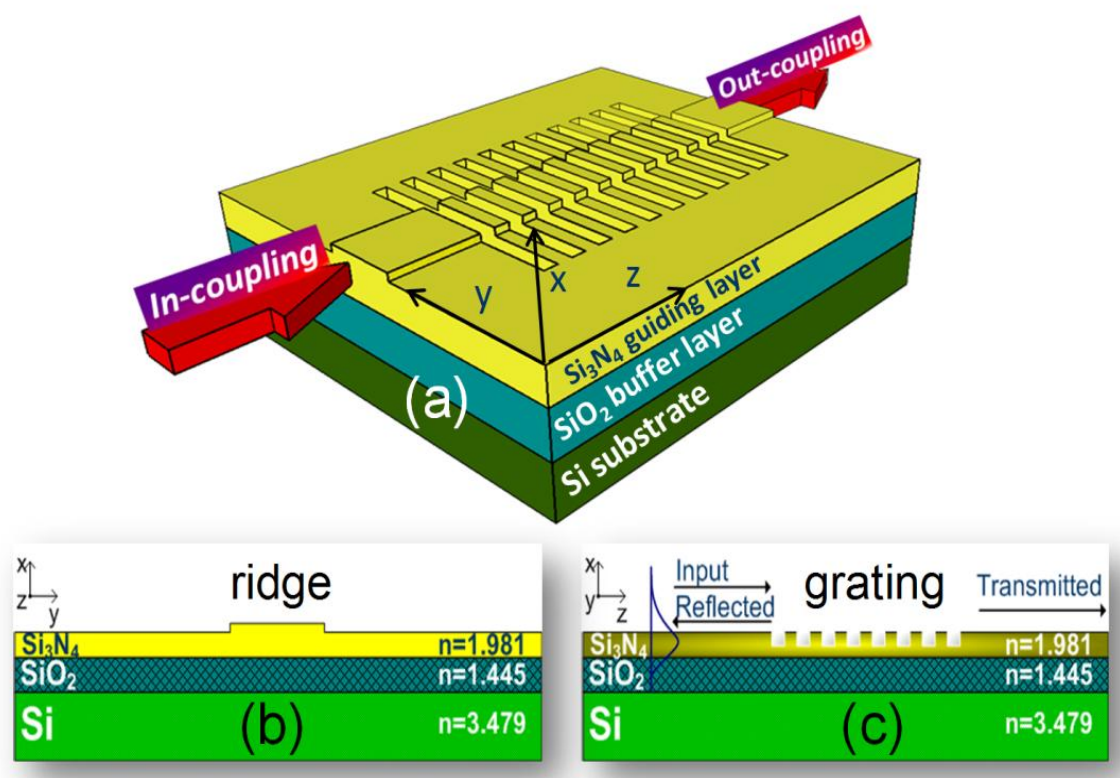

Fig. 2.1 Schematic of a grated waveguide (GWG) with the grated section or grating etched in a shallow ridge waveguide: (a) $3 D$ schematic, and (b) $2 D$ cross-section of the ridge (and teeth of the grating) and (c) $2 D$ cross-section of the grating.

\subsubsection{Theoretical methods for gratings}

The propagation of light in grating has been the subject of many studies and can be simulated by numerical methods, like the Finite Difference Time Domain method (FDTD), the Bi-directional Eigenmode Propagation (BEP) or the Finite Element Method (FEM) [72]. In addition, (approximate) analytical and parametric methods are also relevant for the understanding of the relation between device parameters and performance. In the literature a number of such methods can be found, such as transfer matrix method (TMM) relying on a 1D model [73], or coupled wave method (coupled mode theory) taking into account the grating-induced coupling between forward and backward propagating WG modes [74, 75]. Other (more exact) approaches use Floquet-Bloch modes (the electromagnetic eigenmode solutions of the periodic structure) as a starting point and the electromagnetic fields in a finite periodic structure are expressed as a superposition of two counter propagating Floquet-Bloch modes [76-78]. Derivation of these modes for a periodic grating can be found in details in Refs. [76, 78]. The behavior of light propagating in a GWG cavity (GWCC), i.e., a waveguide 
with a grated section, is indicated in a schematic drawing shown in Fig. 2.2. The length of the grated section is $L=N \times \Lambda$, with $\Lambda$ the period and $N$ the number of periods of the grating. Using for example the grating mode solver (GMS) method presented in Ref. [76], the Bloch modes of the considered GWG can be solved. These can be specified by a free-space wavelength $\lambda$ (or frequency $\omega$ ) and a wave number $k$ (or a propagation constant $\beta$; see Fig. 2.3). Roughly, the GWG mode can be pictured as being the sum of right and left moving WC modes. The amplitudes of the two differ in general considerably but approach each other for GWG modes near the band edge. These GWC modes (in the vicinity of the band edge) are slow-modes with high energy density for given modal power (high $n_{g}$ ).

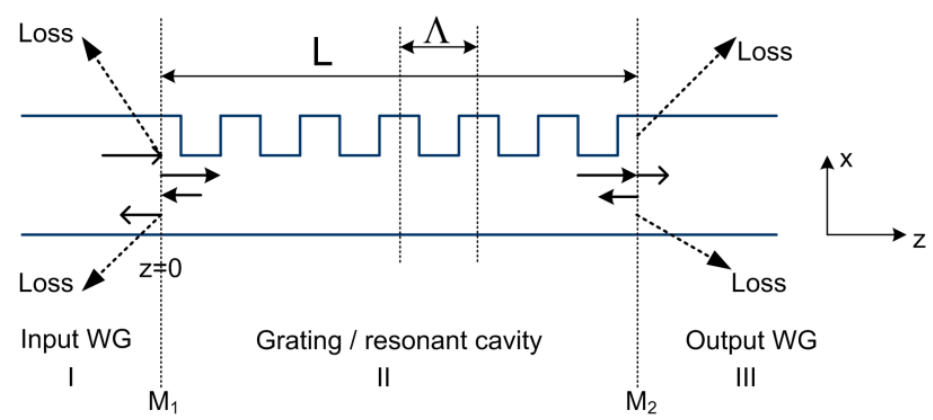

Fig. 2.2 Schematic of light behavior in a GWG.

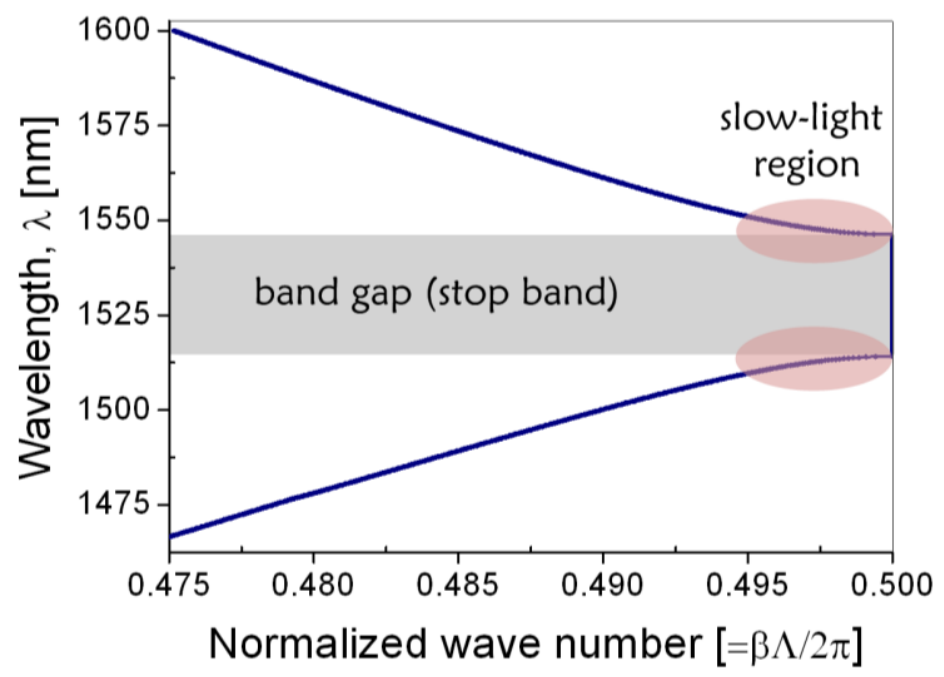

Fig. 2.3 Normalized dispersion curve of a GWG: the flat regions near the band edge correspond to slow modes. 


\subsubsection{Fabry-Perot resonance in a grated waveguide cavity}

In addition to the slow-light effect mentioned above, a finite-length GWC structure also acts as an optical resonator. The two interfaces $\left(M_{1}\right.$ and $\left.M_{2}\right)$ between the input (I)/output (II) WGs and the grated section (III) (see Fig. 2.2) can virtually be treated as the mirrors which form a Fabry-Perot cavity and thus the GWG modes will bounce back and forth in the middle region (III), resulting in multiple reflections. It is noted that a WC mode, coming from the input WG, will be partly reflected, partly transmitted into a grating mode or Bloch mode, and partly scattered into radiation modes. That happens at both interfaces, $M_{1}$ and $M_{2}$. For a perfect structure, i.e., a structure without scattering losses due to possible fabrication imperfections or without material induced absorption losses, the only inevitable losses occur by scattering due to modal mismatch at the transition between the input/output WG and the grated region [76, 79]. Figure 2.4a shows the transmission spectrum of a GWG, showing sharp fringes near the band edges as a result of the Fabry-Perot resonances in the GWCC. The latter correspond to an extra enhancement of the optical field in the GWGC. Figure $2.4 \mathrm{~b}$ zooms in on the dispersion curve of Fig. 2.3. Point A, corresponding to the same-labeled peak in Fig. 2.4a, is located in the slow-light region near the band edge. According to Ref. [76] the $m^{\text {th }}$ resonance below the band edge occurs if the propagation constant satisfies the expression:

$$
\beta_{m}=\frac{\pi}{\Lambda}-m \frac{\pi}{N \Lambda}, m=1,2, \ldots
$$

Equation 2.3 is illustrated by Fig. 2.4b where resonances are indicated for three different lengths of the GWGC. Denoting the propagation constant of the band edge by $\beta_{E}(=\pi / \Lambda)$ it follows that $\beta_{E}-\beta_{A}=2\left(\beta_{E}-\beta_{B}\right)=3\left(\beta_{E}-\beta_{C}\right)(=0.004 \times \pi / \Lambda)$ with $\beta_{A-C}$ corresponding to the lengths $L_{A-C}(=125 \Lambda, 250 \Lambda, 375 \Lambda$, respectively).

The spectral shape of a resonance is usually described by its quality factor $Q$ determined by the energy stored in a resonator at the resonant frequency and the energy loss in one period corresponding to this frequency. In practice, $Q$ is determined by measuring the response (i.e., transmission spectrum) of the resonator. The $Q$ factor can then be obtained using expression [60]: 


$$
Q=\frac{\lambda_{0}}{\Delta \lambda_{-3 d B}}
$$

with $\lambda_{0}$ the resonant wavelength and $\lambda_{-3 \mathrm{~dB}}$ the full width at half maximum (FWHM) of the resonant peak.
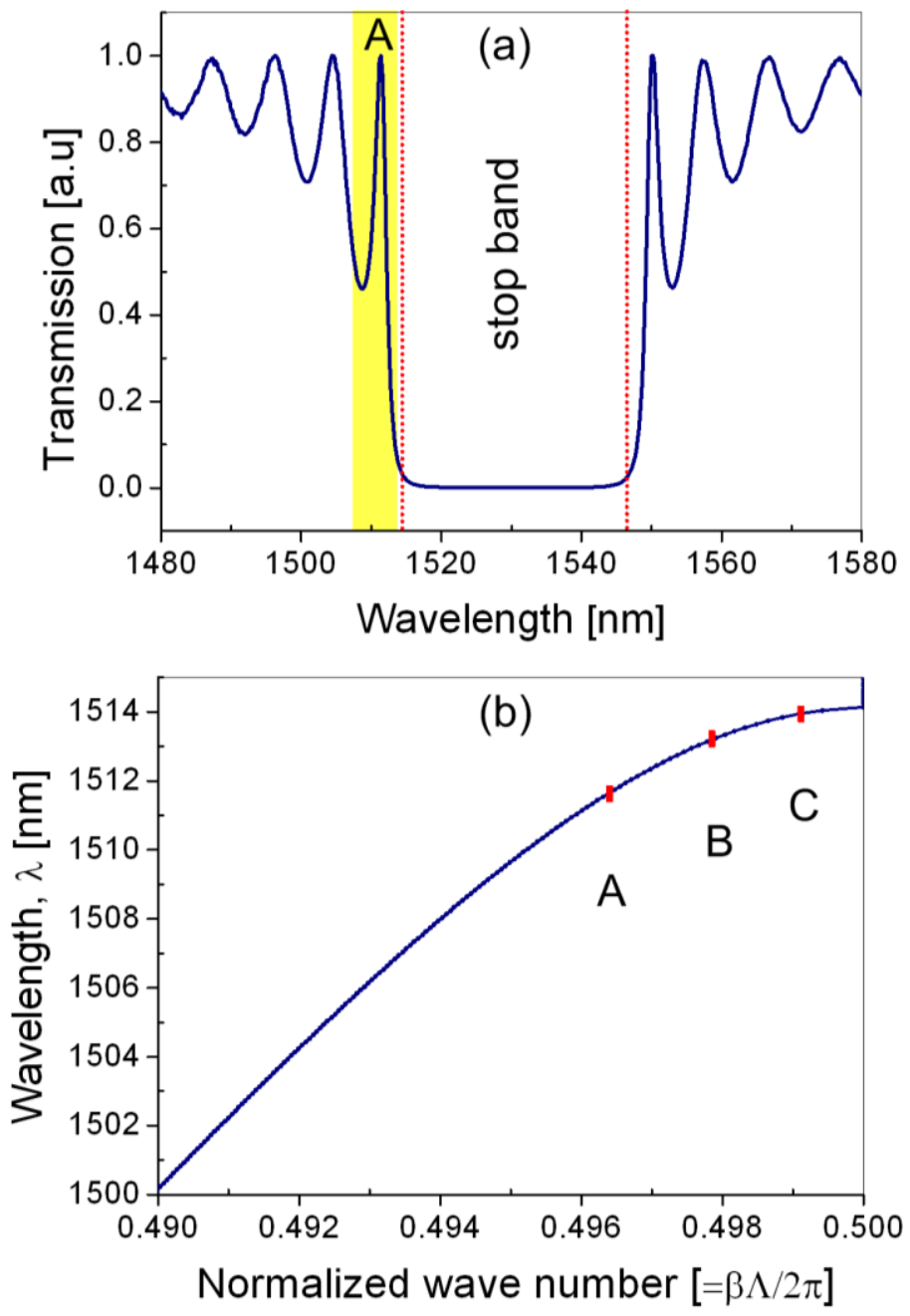

Fig. 2.4 (a) Calculated transmission spectrum of a grating with a length of 125 periods; the highlighted resonant peak $A$ is used for sensing. (b) Zoomed part of the lower branch of the dispersion curve in Fig. 2.3 indicating also the $1^{\text {tt }}$ resonances ( $A, B$ and $C$ ) near the band edge for GWGC lengths of $L_{A}=125 \Lambda, L_{B}=250 \Lambda$ and $L_{C}=375 \Lambda$ and grating depth of $55 \mathrm{~nm}$ (other parameters given in Table 2.1). 


\subsection{Sensing applications and sensitivity analysis}

For integrated optical sensors, there are two commonly used sensing modes: homogenous sensing and surface sensing [19, 21, 32, 80-83]. In addition to these two modes we will consider in this thesis also mechano-optical sensing. For all three sensing modes we consider measurand-induced spectral shifts of the transmittance of a GWGC. These three sensing modes will be applied for chemical sensors, i.e., a bulk concentration, a label-free protein and a gas sensor, respectively (see Fig. 2.5). For chemical sensors their resolution, given as the smallest detectable concentration change, is one of its most relevant performance properties. The value of the resolution or limit of detection, LOD, can be obtained by considering the sensitivity, $S$, and the standard deviation of the different noise and drift terms, as detailed later on in Chapter 3.

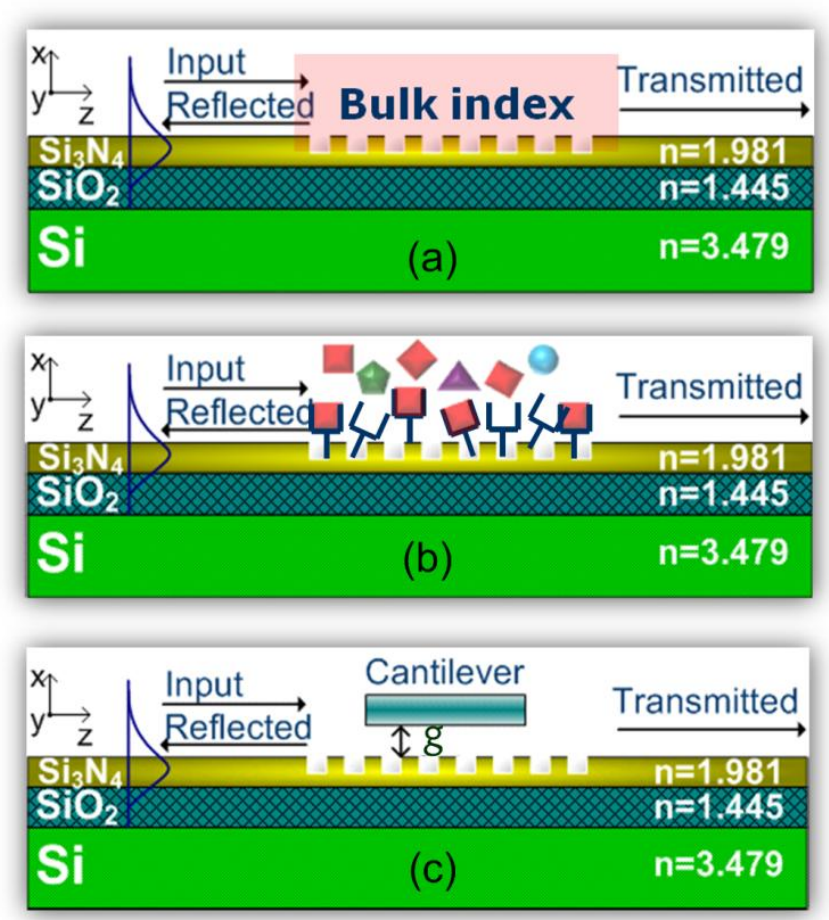

Fig. 2.5 Schematic 2D cross-section of the considered GWG-based devices for (a) bulk, (b) surface, and (c) mechano-optical sensing. 
Sensitivity is generally defined as the derivative of the considered output parameter, say, $B_{\text {out }}$ with respect to the measurand, $M$ :

$$
S_{M}=\left|\partial B_{\text {out }} / \partial M\right| \text {. }
$$

In chemical sensors the measurand is the chemical concentration of the analyte. However a chemo-optical sensing principle is commonly evaluated independently of the specific chemical species by considering the next parameter in the transduction chain, the parameter which is changed as a direct result of this concentration change. In case of bulk sensing it is the refractive index $(n)$ of the homogeneous material (e.g., a solution) in the evanescent field of a propagating mode, in case of surface sensing it is the effective thickness $(h)$ of the thin adlayer which contains receptors for the analyte molecules, in case of mechano-optical sensing it is the gap ( $g$ ) between the grated waveguide and the cantilever suspended above it. In all these cases changes in the index distribution, inducing changes of the propagating GWG mode, are monitored. The three sensing mechanisms can be generalized by the following transduction chain:

$$
\Delta X \rightarrow \Delta \beta(\lambda) \rightarrow \Delta \lambda_{\text {resonance }} \rightarrow \Delta T
$$

where $\Delta X$ denotes a change of the parameter $X(=n, h$, or $g)$, and $\Delta \lambda_{\text {resonance }}$ and $\Delta T$ are the changes of the resonance wavelength and the transmittance, respectively. In case of transmission spectra measurements (as shown in Fig. 2.4a) the sensitivity of the device for changes of $X$ for a given wavelength $\lambda$, is defined as

$$
S_{X} \equiv\left|\left(\frac{1}{T} \frac{\partial T}{\partial X}\right)_{\lambda}\right|=\left|\left(\frac{\partial \ln T}{\partial X}\right)_{\lambda}\right|,
$$

where $T$ is the transmittance. Provided that the detector signal related to $T$ is considerably larger than that of the dark current, most of the noise sources, like laser power fluctuations, cause relative change in the transmittance, therefore $\ln T$ (rather than $T$ ) is considered.

The sensitivity can be rewritten as:

$$
S_{X}=\left|\frac{\partial \ln T}{\partial X}\right|_{\lambda}=\left|\left(\frac{\partial \ln T}{\partial \lambda}\right)_{X}\left(\frac{\partial \lambda}{\partial X}\right)_{T}\right|=\left|\left(\frac{\partial \ln T}{\partial \beta}\right)_{X}\left(\frac{\partial \beta}{\partial \lambda}\right)_{X}\left(\frac{\partial \lambda}{\partial X}\right)_{T}\right|,
$$


where for the second equality we used that $T=T(\lambda, X)^{1}$ and for the third one that the transmittance depends on the laser wavelength $\lambda$ through the propagation constant $\beta$ of the mode via the considered modal dispersion curve $\beta(\lambda)$. A detailed physical interpretation of the terms in Eq. 2.7 is given in Chapter 3 (Section 3.2).

\subsection{Design consideration}

Below we will discuss the choices that have been made for the device parameters.

\subsubsection{Ridge waveguide and grating parameters}

For the design of the GWG device, a ridge waveguide supporting a single TE mode in the infrared wavelength range of $1480-1580 \mathrm{~nm}$ is chosen. Single mode operation is chosen in order to avoid any complication, like unwanted interference between different modes, in the device performance. Fabrication imperfections may cause surface roughness of the grating, which induces scattering losses, i.e., Rayleigh scattering of which the intensity is proportional to $\lambda^{4}$. Therefore, the choice for infrared light leads to lowerloss devices than would be possible with visible light.

$$
\begin{aligned}
& { }^{1} T=T(\lambda, X) \Rightarrow d T=\left(\frac{\partial T}{\partial \lambda}\right)_{X} d \lambda+\left(\frac{\partial T}{\partial X}\right)_{\lambda} d X \Rightarrow \frac{d T}{d X}=\left(\frac{\partial T}{\partial \lambda}\right)_{X}\left(\frac{d \lambda}{d X}\right)+\left(\frac{\partial T}{\partial X}\right)_{\lambda} \\
& T=\text { const } \Rightarrow\left(\frac{d T}{d X}\right)_{T}=0=\left(\frac{\partial T}{\partial \lambda}\right)_{X}\left(\frac{d \lambda}{d X}\right)_{T}+\left(\frac{\partial T}{\partial X}\right)_{\lambda} \\
& \Rightarrow S_{X}=\left|\left(\frac{\partial \ln T}{\partial X}\right)_{\lambda}\right|=\left|-\left(\frac{\partial \ln T}{\partial \lambda}\right)_{X}\left(\frac{d \lambda}{d X}\right)_{T}\right|
\end{aligned}
$$




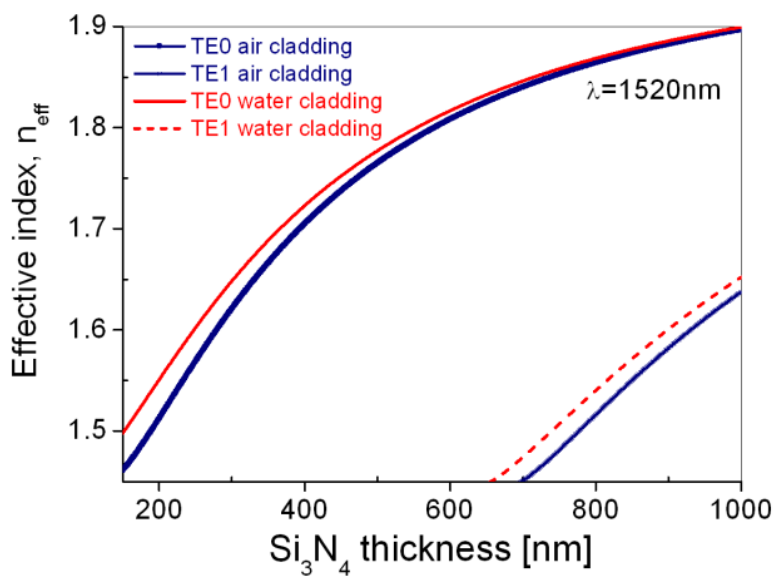

Fig. 2.6 Effective index as a function of the $\mathrm{Si}_{3} \mathrm{~N}_{4}$ guiding layer thickness using a $1 D$ mode solver for a three layer structure, single mode operation is obtained with thickness smaller than $620 \mathrm{~nm}$.

To obtain a single mode ridge waveguide (see Fig. 2.1b), its geometric parameters, i.e., the guiding layer thickness, the width and the height of the ridge, need to be determined. First, using a 1D mode solver for a three layer structure $\left(\mathrm{SiO}_{2} / \mathrm{Si}_{3} \mathrm{~N}_{4} /\right.$ air or water), the upper limit of the $\mathrm{Si}_{3} \mathrm{~N}_{4}$ guiding layer thickness (for single mode) is determined to be $\sim 620 \mathrm{~nm}$ at the wavelength $\lambda=1520 \mathrm{~nm}$ (see Fig. 2.6). However, stoichiometric fabrication of $\mathrm{Si}_{3} \mathrm{~N}_{4}$ limits the grown thickness to $\sim 280-300 \mathrm{~nm}$ due to stress-induced cracks occurring above this thickness [84]. Therefore, a thickness of $275 \mathrm{~nm}$ has been chosen.

A ridge step of $5 \mathrm{~nm}$ height is defined for lateral confinement. Such a small value has the advantage of reducing planarization errors during fabrication and reducing scatter losses due to surface roughness on the edges of the waveguide [85]. In addition, the lateral effective index contrast resulting from the small ridge step is useful for modeling the dispersive properties of the grating sufficiently accurately with a 2D model [60, 72]. With a guiding thickness of $275 \mathrm{~nm}$ and the ridge step of $5 \mathrm{~nm}$, a width up to $8 \mu \mathrm{m}$ can be chosen for the ridge waveguide to be still single mode in horizontal direction (in the infrared wavelength range of 1480-1580 nm). To give room for fabrication errors, a ridge width of $5 \mu \mathrm{m}$ is chosen.

Given the channel structure, the spectral position of the relevant fringe is determined by the grating period and depth. Based on the design guidelines 
presented in Ref. [60], the grating parameters are chosen as follows: the period $\Lambda=490 \mathrm{~nm}$ with a duty cycle (width-ratio between a groove and a tooth) of $\sim 50 \%$, and the grating depth of $55 \mathrm{~nm}-75 \mathrm{~nm}$. Such a choice is for obtaining a wavelength for the first band gap, both with air and water cladding, within the tuning range of the laser $(1450-1600 \mathrm{~nm})$. The GWC parameters are summarized in Table 2.1.

Table 2.1 Fabrication parameters of the GWG structure shown in Fig.2.1.

\begin{tabular}{ll}
\hline Parameter & Value \\
\hline $\mathrm{Si}_{3} \mathrm{~N}_{4}$ guiding thickness & $275 \mathrm{~nm}$ \\
Ridge height & $5 \mathrm{~nm}$ \\
Ridge width & $5 \mu \mathrm{m}$ \\
Grating period & $490 \mathrm{~nm}$ \\
Duty cycle & $\sim 50 \%(245 \mathrm{~nm} / 245 \mathrm{~nm})$ \\
Number of period & 250 \\
Grating depth & $55-75 \mathrm{~nm}$ \\
\hline
\end{tabular}

\subsubsection{Mechanical structure parameters}

In the GWG-CL integration structure for mechano-optical sensing application, the parameters of the CWG structure remain the same as used for homogeneous and surface sensing. The parameters of mechanical structures (i.e., singly clamped cantilever and doubly clamped cantilever (bridge)) are discussed in Chapters 4 (experiments) and 6 (optimization).

\subsection{Conclusion}

This chapter presents the characteristics of slow-light propagating in a GWG structure. A WG with a grated section acts as an optical resonator showing sharp fringes near the band-edge of the stopband, adjacent to which the slow-light regions are situated. Three sensing applications are introduced, which make use of the GWG as platform for bulk-index concentration, direct and label-free protein, and GWG-CL integrated mechano-optical sensors. The sensitivity $(S)$ of the sensors is defined and device design considerations are also discussed in short. 


\section{Bulk-index concentration and direct, label-free protein sensing}

A theoretical and experimental evaluation is given of a principle of direct, label-free opto-chemical sensing. According to this principle, which is applicable in compact integrated optical sensors, measurand-induced wavelength shifts of the sharp fringes in the transmission spectra near the stop band edges of a resonant grating-based cavity are monitored. Such fringes are the results of Fabry-Perot resonances of the Bloch modes propagating in the cavity. Two sensor configurations have been considered, a first one for measuring the concentration of a single compound dissolved in water in the vicinity of the grating (bulk sensing), and a second one for determining the concentration of a specific compound adsorbed at the grating surface from a watery mixture of many compounds (surface sensing). In the latter, a thin interface layer which contains receptors specific to the targeted analyte, the PepN enzyme, is applied on top of the grated waveguide section. Filling of the receptors can be effectively seen as growth of an adlayer. Experimentally resolutions of $6 \times 10^{-6}$ RIU and $\sim 4 \mathrm{pm}$ adlayer growth have been obtained for bulk and surface sensing, respectively. With a statistical analysis the limitations to obtain lower resolutions with the current set-up are identified. The compact devices (footprint $\sim 200 \times 15 \mu \mathrm{m}^{2}$ ) are well suited for multi-sensing in lab-on-a-chip systems and can easily be fabricated with standard micro-fluidic and CMOS technologies. ${ }^{2}$

2 Part of this chapter has been submitted for publication as: So V. Pham, Meindert Dijkstra, Anton J. F. Hollink, Lasse J. Kauppinen, René M. de Ridder, Markus Pollnau, Paul V. Lambeck and Hugo J. W. M. Hoekstra, "On-chip bulk-index concentration and direct, label-free protein sensing utilizing an optical gratedwaveguide cavity," (2012). 


\subsection{Introduction}

In recent years monolithically integrated optical resonant cavities, such as Bragg-grating-based Fabry-Perot resonators, microspheres, microdisks, microtoroids, photonic crystal cavities, and ring resonators, have been exploited as high-sensitivity platforms for refractive index and label-free molecular sensing [16, 17, 21, 83, 86-88]. In the Bragg-grating-based resonators a grated waveguide (GWG), which is a waveguide (WG) with a finite-length grated section, reflects incoming modes in a narrow wavelength region, defined by the geometry of the grating and the refractive indices of the constituting materials. Generally, the measurand-induced wavelength shift of the complete reflection band, the so-called stop band, is measured by monitoring the reflected light. In the sensor studied here, however, we measure the shift of a sharp fringe in the transmission spectrum near the stopband edge of the grating. These fringes are due to Fabry-Perot resonances of Bloch modes propagating in the cavity defined by the grated section [59, 60]. Any small perturbation in the environment of the GWG, which affects the modal fields of the GWC modes, will lead to a wavelength shift of its transmission spectrum. Such an effect can be exploited for sensing applications. In 2005, our group fabricated and demonstrated an optical GWG cavity as a read-out for bulk-index evanescent-field sensing with a resolution for index changes of $\Delta n \cong 4 \times 10^{-4}$ in the visible wavelength range [59]. As a follow-up, we fabricated and demonstrated a low-loss GWC for measuring nano-displacements of a cantilever suspended above the GWG [89] and mechano-optical hydrogen gas sensing [90]. In this chapter, we demonstrate two applications of the optical GWG cavity: (1) a chemical concentration sensor, based on a homogeneous index change within the evanescent field of the GWG top cladding (bulk sensing), affording a resolution of $6 \times 10^{-6} \mathrm{RIU}$; (2) a direct, label-free protein biosensor (e.g., PepN enzyme - the major Suc-LLVY-AMC-hydrolyzing enzyme in Escherichia coli), where the GWG spectral shift is due to an antigen-antibody interaction leading to the effective growth of an adlayer on top of the core layer of the cavity (surface sensing), affording a resolution of $4 \mathrm{pm}$ adlayer growth.

This chapter is organized as follows. In section 3.2 the used device structure and its working principle are introduced. Sensitivity and noise/drift aspects are discussed, directed towards obtaining expressions for the resolution. In section 3.3 the design of a specific GWG device is discussed and 
the peripheral equipment is described. In section 3.4 and 3.5 experimental results for both bulk and surface sensing are given and discussed, respectively. Conclusions are drawn in section 3.6.

\subsection{Device structure and sensitivity analysis}

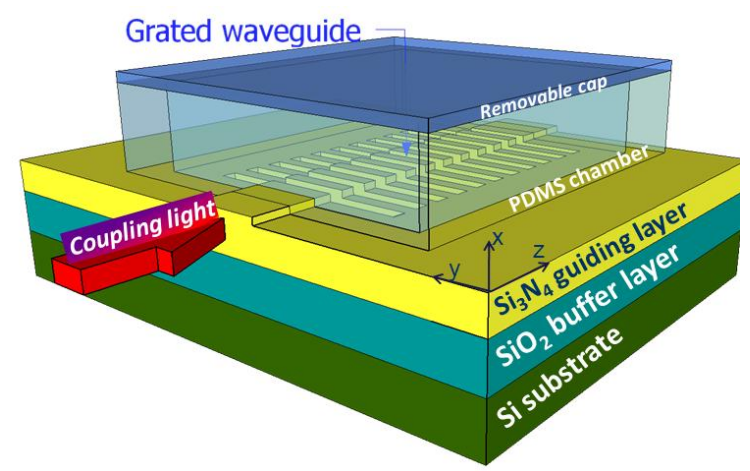

Fig. 3.1 A 3D schematic structure of the GWG device

A 3D schematic structure of the GWG device is shown in Fig. 3.1. The crucial part of the device is the grating section etched into the $\mathrm{Si}_{3} \mathrm{~N}_{4}$ core layer of the mono-modal waveguide. Any small change of the refractive index distribution within the evanescent field of the guided mode will result in a wavelength shift of its transmission spectrum. Such a change can be due to a change in the analyte concentration in a solution flowing over the grating section inside the covering PDMS chamber. Such a transmission spectrum, calculated by use of appropriate software (see section 3.3), is displayed in Fig. 3.2. The peaks in Fig. 3.2 correspond to resonances in the Fabry-Perot cavity defined by the transition between WG and GWG sections. At these transitions modal mismatch leads to modal reflection and so to the wellknown oscillations in the transmittance of which the peaks correspond to resonant excitation of the cavity. The grating has been designed such that the feature of the transmission band of which the shift is to be measured, in this case the sharp peak (fringe) highlighted in the figure, is within the wavelength range of the tunable laser to be used for probing the transmission spectrum. 


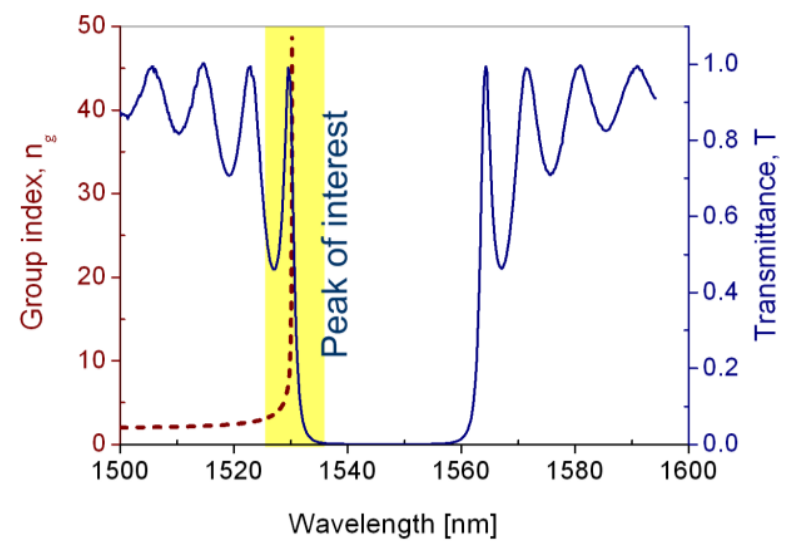

Fig. 3.2 Typical transmission spectrum (calculated, solid line) of the GWC structure; any small structural changes (bulk or surface) within the GWG modal evanescent field will result in its spectral shift; the sharpest peak (peak of interest) is used for sensing. The dashed line indicates the calculated group index (see Section 3.3).

The wavelength shift of a spectral feature can be monitored by measuring either a relevant part of the transmission spectrum or transmission variations at a constant wavelength, preferentially where the slope of the spectrum is at its maximum. For the considered sensor technological control is insufficient to position (at a given refractive index distribution) the point of steepest slope of the narrow peak at a predefined laser wavelength. Consequently, in both methods a tunable laser must be applied. Owing to noise or drift effects (e.g., in laser power) we have chosen to monitor spectral shifts of the peak of interest.

The resolution of a chemical sensor is defined as the smallest measurable concentration change. The value of the resolution, or limit of detection (LOD), depends on the sensitivity, $S$, and the standard deviation (SD) of the different noise and drift terms. The sensitivity is the derivative of the considered output parameter, $B_{\text {out }}$, and the measurand, $\mathcal{M}$ :

$$
S_{M}=\left|\partial B_{\text {out }} / \partial M\right|
$$

In chemical sensors the measurand is the chemical concentration of the analyte. However, the sensor principle is generalized by considering an effective measurand, the parameter which is altered as a direct result of this concentration change. In our case of bulk sensing it is the refractive index of 
the solution in the evanescent field of the propagating mode, in our case of surface sensing the effective thickness of the thin adlayer which contains receptors for the analyte molecules.

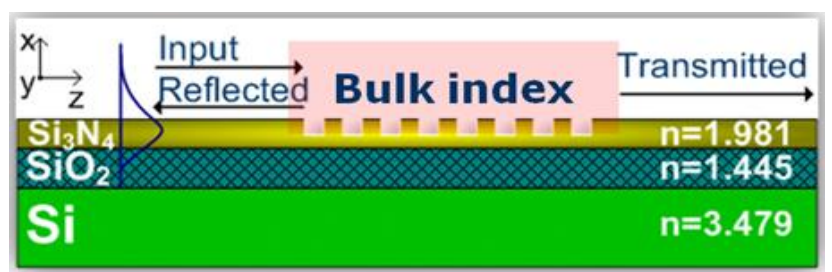

Fig. 3.3 Schematic 2D cross-section of the device for bulk concentration sensing.

In the following we will analyze theoretically the resolution for both readout methods, bulk and surface sensing. As discussed in Chapter 2 (Section 2.3), the expression for sensitivity is repeated here for clarity and convenience. For bulk sensing (see Fig. 3.3) using transmission spectra measurements, the sensitivity for a given wavelength $\lambda$ is defined as [91]

$$
S_{n} \equiv\left|\frac{1}{T} \frac{\partial T(\lambda, n)}{\partial n}\right|_{\lambda}=\left|\frac{\partial \ln T}{\partial n}\right|_{\lambda}
$$

where $T$ is the transmittance and $n$ is the homogeneous refractive index in the evanescent field region of the grating. Provided that the detector signal related to $T$ is considerably larger than that of the dark current, most of the noise sources, like laser power fluctuations, cause relative changes in the transmittance, therefore $\ln T$ (rather than $T$ ) is considered.

The sensitivity can be rewritten as:

$$
S_{n}=\left|\frac{\partial \ln T}{\partial n}\right|_{\lambda}=\left|\left(\frac{\partial \ln T}{\partial \lambda}\right)_{n}\left(\frac{\partial \lambda}{\partial n}\right)_{T}\right|=\left|\left(\frac{\partial \ln T}{\partial \beta}\right)_{n}\left(\frac{\partial \beta}{\partial \lambda}\right)_{n}\left(\frac{\partial \lambda}{\partial n}\right)_{T}\right|,
$$

where for the second equality we exploited that $T=T(\lambda, n)$ and for the third one that the transmittance depends on the laser wavelength $\lambda$ through the propagation constant $\beta$ of the optical mode via the considered modal dispersion curve $\beta(\lambda)$.

The three factors on the rightmost side of Eq. 3.3 enable a simple physical interpretation of what is important to attain a high $S_{n}:(\partial \lambda / \partial n)_{T}$ is 
proportional to the fraction of the squared electric modal field of the grating in the cladding [92] (equations 28 and 29 of chapter 2 of Ref. [92]); $\partial \beta / \partial \lambda$ is inversely proportional to the group velocity, $v_{g}$, and $\partial \ln T / \partial \beta$ (rather than $\partial \ln T / \partial \lambda$, for highly dispersive systems) reflects the interferometric device read-out principle (here Fabry-Perot interferometry). All these three quantities are measures for interaction between the light and the matter to be sensed. The group velocity $v_{g}$ is equal to the ratio of optical power and energy density, both integrated over the cross-section of the uniform WC or the volume of the unit cell of a CWG [93]. Consequently, a large value of $\partial \beta / \partial \lambda$ (which occurs near band edges) indicates strong lightmatter interaction and low $v_{g}$ values. The quantity $\partial \lambda / \partial n$ plays only a minor role in optimizing sensor structures, because it can be only slightly increased by optimizing the core layer thickness, unlike the quantity $\partial \ln T / \partial \beta$ which may grow by orders of magnitude in resonant structures and the group velocity, $v_{\mathrm{g}}$, which under certain conditions will become small. Owing to the low modal group velocity and also to group delay in the cavity these resonator devices are often called slow light devices. To illustrate this, Fig. 3.2 also shows the modal group index $n_{g}\left(\equiv c / v_{g}\right)$ as a function of $\lambda$ in the relevant wavelength region. The curves have been calculated for the practically realized grating system, of which the parameters will be given in section 3.3.

In this sensor the sources of noise and drift can be divided into two types: sources which are identically effective in absence of a grating and those which are especially related to the measured wavelength position of the fringe. To the first type belong fluctuations in output power of the light source, variations in coupling efficiencies to and from the optical chip and noise generated in the detection system. Of the second type are effects owing to fluctuations of the temperature, $\delta \tau$, and the uncertainty in the wavelength of the laser, $\delta \lambda$, in a tunable laser given as the wavelength repeatability.

Part of these sources, such as fluctuations of the light source power and responsivity of the detector, lead to noise proportional to $\ln T$, implying a noise contribution to $\ln T$ with a constant SD as a function of scanning wavelength. Other noise terms, such as temperature or scanning wavelength variations, contribute to a non-constant SD of $\ln T$ according to, e.g.,

$$
\sigma_{\ln T, \lambda}=|\partial \ln T / \partial \lambda| \sigma_{\lambda} \text {. }
$$


Here $\sigma_{\ln T, \lambda}$ denotes the SD of $\ln T$, as caused by scanning wavelength fluctuations, and $\sigma_{\lambda}$ is the SD of the scanning wavelength. In the discussion further on we will consider only approximate values of the SDs and LODs. For this reason, and also for simplicity, we will use an (estimated) average of terms like $\sigma_{\ln T, \lambda}$ and assume that these are constant over the considered wavelength range. Assuming also a normal noise distribution for such terms and no correlation between these terms, it follows from standard statistics that

$$
\sigma_{\ln T, t o t}=\sqrt{\sigma_{\ln T}^{2}+\sigma_{\ln T, 1}^{2}+\sigma_{\ln T, 2}^{2} \cdots},
$$

where the subscripts $1,2, \ldots$ indicate the noise sources (like $\lambda$ in Eq. 3.4).

If during sensing peak shifts are determined using $P$ data points, the $L O D_{n}$ can be expressed as [94]

$$
L O D_{n} \approx \alpha \sigma_{\ln T, t o t} /\left(S_{n, \max } \sqrt{P}\right),
$$

with $S_{n, \max }$, the maximum sensitivity along the spectral curve, and $\alpha$, a correction factor of around 2 to 4 [91], which accounts for the fact that data points are used with varying $S_{n}$, the shape of the spectral response curve and the way data are processed. Below we will present a way to determine this quantity numerically. The factor $1 / \sqrt{P}$ expresses the dependence of the LOD on the number of data points. To arrive at Eq. 3.6, normal, uncorrelated noise was assumed.

For later use we note that, similarly as above, one may derive an equation for $L O D_{\lambda}$ which relates to $L O D_{n}$ as follows:

$$
L O D_{\lambda} \approx \alpha \sigma_{\ln T, t o t} /\left(S_{\lambda, \max } \sqrt{P}\right)=L O D_{n}\left|(\partial \lambda / \partial n)_{T}\right|=\alpha \sigma_{\lambda, t o t} / \sqrt{P},
$$

with $S_{\lambda, \max }\left(\equiv|\partial \ln T / \partial \lambda|_{n, \max }\right)$ defined similarly as $S_{n, \max }$. The last equality, with $\sigma_{\lambda, t o t} \equiv \sigma_{\ln T, t o t}\left|(\partial \lambda / \partial \ln T)_{n}\right|$, is for later use.

From the above we can see that, in order to minimize the $L O D_{n}$, one has to minimize all terms occurring at the right hand site of Eq. 3.5, including those caused by the peripheral equipment, and maximize the sensitivity as well as the number of data points $P$. 
In order to obtain an approximate value for $\alpha$ and also to check the form of Eqs. 3.6 and 3.7 numerical experiments have been performed as follows. We first considered Eq. 3.7. As a starting point we used an arbitrary, experimental spectral response curve (of the considered peak). With spectral filtering, as described below, a smooth, quasi-noise-free curve $\ln T$ versus $\lambda$ was obtained. Normal noise was synthetically added at a fixed value of $\sigma_{\ln T, t o t}$ a large number (500) of times, after which the noise-induced spectral peak shifts (compared to the quasi-noise-free peak) were determined. The SD of the results defines the $L O D_{\lambda}$. This process was repeated for different values of $\sigma_{\ln T, t o t}, S_{\lambda, \max }$ (by varying the width of the response curve maintaining its shape) and $P$ (by removing in a regular way, a fraction of the data points). It was found that $\alpha \sim 3.2$ and that the values for $L O D_{\lambda}$ obtained in this manner were indeed proportion to $\sigma_{\ln T, t o t} /\left(S_{\lambda, \max } \sqrt{P}\right)$. Owing to the simple relation between $L O D_{\lambda}$ and $L O D_{n}$ (see Eq. 3.7) it now follows that also Eq. 3.6 is of the correct form.

Similarly as for bulk sensing we can introduce a sensitivity for surface sensing,

$$
S_{h} \equiv\left|\left(\frac{\partial \ln T}{\partial \lambda}\right)_{h}\left(\frac{\partial \lambda}{\partial h}\right)_{T}\right|,
$$

with $h$, the thickness of the adlayer grown by the antigen-antibody interaction. A theoretical expression for $(\partial \lambda / \partial h)_{T}$ can be derived from Eq. 30 in chapter 2 of Ref. [92], but its value can also be evaluated using a grating mode solver (GMS) [76].

For surface sensing an additional source of error appears: a change of the refractive index of the solution above the adlayer. However, the time scale of shifts due to addition of the solution and shifts due to the capture of the analyte molecules are, in general, so different that this disturbance can be swept out easily.

Effects of noise in sensing systems can be reduced by filtering out high frequency components of the measured signal (here $\ln T$ versus $\lambda$ ). This filtering includes the removal of not yet mentioned parasitic Fabry-Perot oscillations originating from the resonator formed by, e.g., the end facets of the chip. The filtering process [94] we will apply consists of multiplying the 
discrete Fourier transform with a flat-top function equal to zero, except in a small frequency region around zero frequency where its value is equal to unity. The frequency width of this region is chosen (by visual inspection) to be slightly larger than that corresponding to the width of the response peaks.

\subsection{The sensing system}

Starting point of the design of the optical chip is the requirement that the spectral position of the fringe of which the spectral shift is to be measured must be inside the tuning range of an appropriate, inexpensive tunable laser. Such lasers are available with a tuning range of around 1450-1600 nm, as developed for long-haul optical telecommunication.

The GWG structure is shown in Figs. 3.1 and 3.3. The permanent layers are the bottom $\mathrm{SiO}_{2}$ cladding (thermally oxidized, $\mathrm{n}=1.445$ ) and the $\mathrm{Si}_{3} \mathrm{~N}_{4}$ core layer (LPCVD, $\mathrm{n}=1.981$ ).. The channel and grating structure are etched into the $\mathrm{Si}_{3} \mathrm{~N}_{4}$ core layer. The refractive indices refer to $1500 \mathrm{~nm}$ wavelength. These materials show low optical losses in this wavelength region and such structures can be easily realized with good technological control by standard procedures.

The precise GWG structure, for application in bulk sensing, has to fulfill several requirements:

* mono-modality for TE light in the wavelength region 1450- $1600 \mathrm{~nm}$,

* it should enable efficient coupling of laser light into the waveguiding structure,

* the structure of the grating must permit that the fringe to be monitored is within the tuning range of the tunable laser,

* a large sensitivity has to be obtained by exploiting Eq. 3.3.

By use of slab solving simulation software it can be shown that the $\mathrm{Si}_{3} \mathrm{~N}_{4}$ layer thickness which provides maximum power fraction in the evanescent field in the presence of a water cladding in the sensing section is somewhat larger than $275 \mathrm{~nm}$, which is the maximum thickness which can be obtained without layer cracking. When choosing this thickness value, the minimum thickness of the $\mathrm{SiO}_{2}$ buffer layer for avoiding penetration of the modal field into the Silicon wafer is about $5 \mu \mathrm{m}$.

A $5 \mu \mathrm{m}$ wide ridge-type channel (ridge height $5 \mathrm{~nm}$ ) was obtained by wet etching. This channel structure allows an acceptable coupling efficiency of $9 \%$ from the pig-tailed laser [89]. Given the channel structure and the 
groove depth, the spectral position of the relevant fringe is completely determined by the period of the grating. For obtaining a wavelength, both with air and water cladding, within the tuning range of the laser a grating period of $490 \mathrm{~nm}$ was chosen. The grating was produced by laser interference lithography and dry etching. The groove depth $(75 \mathrm{~nm})$ as well as the length $(122.5 \mu \mathrm{m})$ of the GWG section are important parameters for the device sensitivity, but have not been optimised, as the presented research aims mainly at a proof of principle. Details of the fabrication process can be found elsewhere [90].

With the complete structure being defined, transmission curves and modal dispersion curves can be calculated. These calculations are done using a Grating Mode Solver (GMS) [76] and the bi-directional eigenmode propagation (BEP) method [60,95]. Figure 3.4a displays the peak positions as calculated with the GMS (solid curve), starting with an air cladding and then increasing the cladding index in 0.05 steps to 1.350 , somewhat larger than the refractive index of water, 1.312 at $1500 \mathrm{~nm}$ [96]. From these data a theoretical sensitivity of the peak shift for cladding index changes of $(\partial \lambda / \partial n)_{T}=140 \mathrm{~nm} /$ RIU (Refractive Index Unit) is derived. Cood correspondence between the two calculation methods and reasonable agreement of the two with experimental results with air and water as cladding is obtained.

The maximum sensitivity for the device with water cladding, can now, with Eq. 3.2, be determined from $(\partial \lambda / \partial n)_{T}=140 \mathrm{~nm} / \mathrm{RIU}$ and the maximum experimental value for $\partial \ln T / \partial \lambda$, being $5.7 / \mathrm{nm}$, leading to $S_{n}=800 / \mathrm{RIU}$.

As to the peripheral equipment, a pig tailed tunable laser (Agilent 8164B) with a tuning range of $\sim 1450-1600 \mathrm{~nm}$ has been used as light source. This laser shows a minimal step size of $0.1 \mathrm{pm}$, a wavelength uncertainty (repeatability) of $0.3 \mathrm{pm}$ and a power flatness of $0.25 \mathrm{~dB}$ per $190 \mathrm{~nm}$. Detection of the transmitted power is performed using an InGaAs detector, followed by a signal-processing system, that finally delivers a $\log T$ (in $\mathrm{dBm}$ ) versus $\lambda$ curve from which the position of the maximum of the first peak at the lower wavelength side of the stop band can be derived. The processing includes the filtering procedure described earlier, as well as averaging of the data points of three scans, each taking 7 seconds; the time in between two such scans was typically $60 \mathrm{~s}$. The wavelength of the considered peak was determined by calculating the centre of gravity (or the first moment), $\lambda_{p}$. For 
obtaining highest accuracy it appeared to be optimal not to consider the $\ln T$ spectrum itself, but the $T^{q}$ spectrum (with $q \sim 4$ ), because in that case the peak is well separated from the rest of the spectrum, having $T^{q} \sim 0$ regions left and right of the resulting curve, according to:

$$
\lambda_{p}=\sum_{i=1}^{P} \lambda_{i} T_{i}^{q} / \sum_{i=1}^{P} T_{i}^{q} .
$$

In the above, $P$ is the number of used data points.

The grating section is provided with a removable PDMS chamber. The chip is mounted on top of a Peltier element and, by use of a feedback loop, the temperature can be held constant at $21.5^{\circ} \mathrm{C}$ within $0.03^{\circ} \mathrm{C}$. The influence of temperature and also scanning wavelength errors can be reduced by adding an (nearly) identical reference GWG on the chip, close to the sensing GWG and by monitoring relative wavelength shifts on sensing.

\subsection{Bulk concentration sensing: results and discussion}

Experimentally, we monitored the wavelength position of the relevant fringe of the fabricated GWG with two different claddings, air and water. For both systems the experimentally obtained transmission spectrum is given in Fig. 3.4b. The signal in the stopband is here more than 1000 times lower than outside this band, implying that the influence of stray light is very small. The fact that the grooves are well filled with water (in the case of a water cladding) without significant air bubbles is evidenced by the dashed curve in Fig. 3.4a, which shows the calculated shift for the case that even in a watery environment the grooves are completely filled with air. 

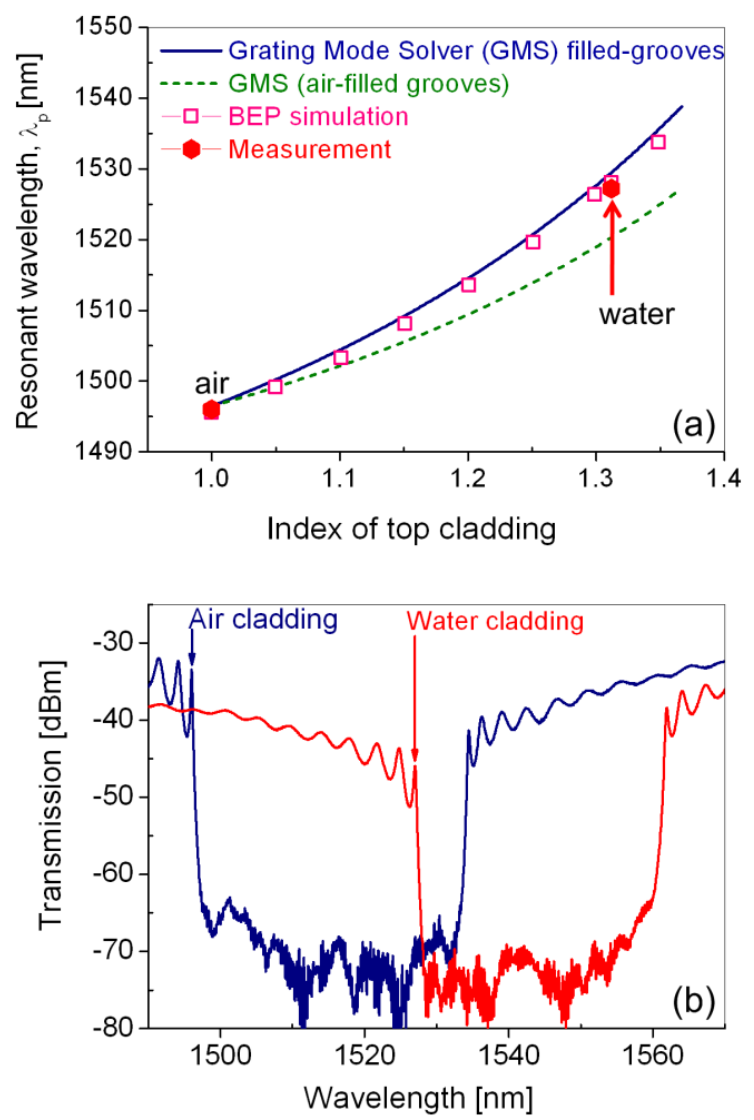

Fig. 3.4 (a) Simulated and measured resonant wavelengths as a function of refractive index of top cladding. The dashed line displays calculated results assuming air-filled grating grooves. (b) Transmission spectra with air and water cladding (water-filled grooves).

For further bulk concentration sensing experiments an open chamber (cuvette) on the surface of the sensor was filled with a phosphate buffered saline solution ( $1 \mathrm{wt} . \% \mathrm{PBS}$ ). The evaporation of water from the open cuvette leads to a continuous change of concentration and, hence, a change of the distribution of the bulk index. This can be clearly seen in the continuous wavelength shift of the fringe. A few filtered spectra are shown in Fig. 3.5a. As the water in the PBS solution evaporates during 42 minutes, the fringe peak wavelength changes from $1521.074 \mathrm{~nm}$ to $1521.950 \mathrm{~nm}$, indicating an index change of 0.006 and, assuming a linear relation between concentration and index for PBS in water, a concentration change from 1 to 5.23 wt.\% (see Fig. 3.5b). 

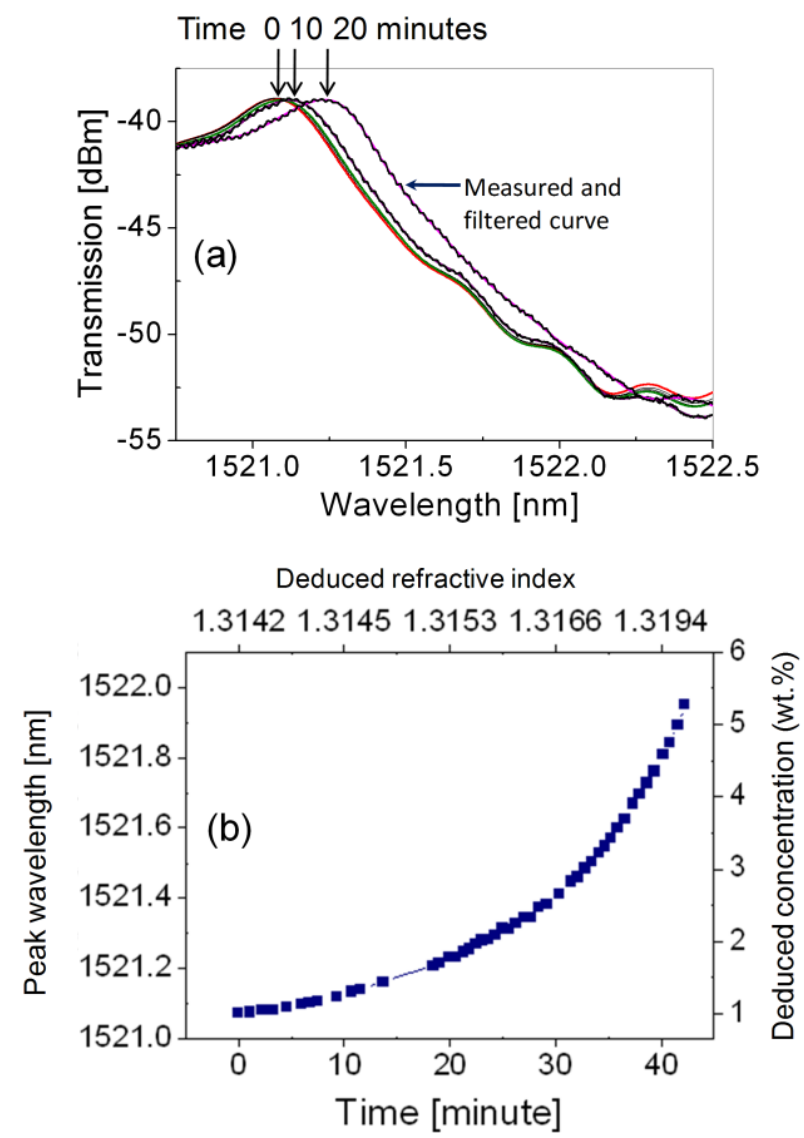

Fig. 3.5 Transmission spectra of the concentration sensor, and (b) wavelength shifts and deduced concentrations due to changes of refractive index as a function of time.

Next we will determine the $L O D_{n}$ discussed in section 3.2. By measuring the filtered transmission spectra of the device continuously during $\sim 30$ minutes under constant sensing conditions it was found that the SD in the peak position is $L O D_{\lambda}=0.8 \mathrm{pm}$. Thus, also using the experimentally obtained value of the sensitivity, $140 \mathrm{~nm} / \mathrm{RIU}$, it follows for the resolution or, alternatively, the LOD (see Eq. 3.7) a value of $L O D_{n}=6 \times 10^{-6}$, implying that PBS-concentration changes down to $0.005 \mathrm{wt} . \%$ can be resolved.

By also measuring the $L O D_{\lambda}$ of unfiltered curves it was concluded that filtering hardly reduces the noise. This indicates that the errors are mainly caused by the slow temperature and/or scanning wavelength variations. Owing to being slow these variations remain almost constant during 
measuring one response curve, which implies correlation between used data points such that Eqs. 3.6 and 3.7 no longer hold, and the LOD reduction is not proportional to $1 / \sqrt{P}$.

Assuming that the error is mainly determined by the uncertainty in temperature $(\delta \tau= \pm 0.03 \mathrm{~K})$ and wavelength (repeatability $\sigma_{\lambda, \text { rep }}=0.3 \mathrm{pm}$ ), we can find, assuming uncorrelated noise, an expression for $L O D_{\lambda}$ from the total SD of the scanning wavelength $\left(\sigma_{\lambda}\right)$ as follows:

$$
\sigma_{\lambda, \text { tot }}=\sqrt{\left(\sigma_{\lambda, \text { rep }}\right)^{2}+\left(\sigma_{\lambda, \tau}\right)^{2}}=0.57 \mathrm{pm},
$$

where we used $\sigma_{\lambda, \tau}=\partial \lambda / \partial \tau \times \delta \tau$ with $\partial \lambda / \partial \tau=16 \mathrm{pm} / \mathrm{K}$, as measured for the system with air cladding (presented in Section 5.3). Next, also using Eq. 3.7 we arrive at:

$$
L O D_{\lambda}=\alpha \sigma_{\lambda, t o t} / \sqrt{P}=0.08 \mathrm{pm},
$$

with $\alpha=3.2, \quad \sigma_{\lambda, t o t}=0.57 \mathrm{pm}$, and $P \approx 500 . L O D_{\lambda}=0.08 \mathrm{pm} P \approx 500$. This theoretical value of $L O D_{\lambda}=0.08 \mathrm{pm}$ is much smaller than the experimental value of $0.8 \mathrm{pm}$, which we attribute to the presence of slow, correlated noise as mentioned above.

Due to the presence of such slow noise, a structural improvement (to increase the factor $\partial \ln T / \partial \lambda$ ) would not improve the resolution of the set-up. With given peripheral equipment only a different waveguide lay-out to increase the spectral shift per RIU $\left(\partial \lambda_{p} / \partial n\right)$ may contribute to improvement of the resolution. Of course, reducing the noise by improving the peripheral equipment (meaning a reduction of the temperature fluctuations and the use of a tunable laser with better repeatability) will improve the resolution.

\subsection{Label-free protein sensing}

\subsubsection{Basics of label-free protein sensing}

For the detection of biological materials (DNA or protein), there is one condition that the detection should be specific. The target DNA or protein should be recognized by the detection method selectively and specifically out of numerous other DNAs or proteins in crude samples (e.g., samples extracted from cells). To detect protein using a biosensor or a biochip, 
traditionally people rely on antigen-antibody interaction (or immunological reaction). The process comprises 2 main steps:

(1) Immobilization of antibody ( $A b)$ onto the reacting area of the chip. Depending on the surface chemistry of the chip, the $A b$ can be immobilized by means of physical adsorption via hydrophobic interaction, self-assembled monolayers, or covalent coupling [17]. In this thesis, the sensor is made of silicon nitride $\left(\mathrm{Si}_{3} \mathrm{~N}_{4}\right)$, which is not a typical material for an $A b$ to be immobilized directly. Therefore a surface functionalization is needed, consisting of 2 steps, i.e., surface activation with silanization and modification with glutaraldehyde.

(2) Once the $A b$ is immobilized and the blocking/washing/drying step is sequently performed, a cell-free extracted solution (containing many kinds of proteins) is applied. If the antigen (Ag) of interest is present, it will be bound to the $A b$ on the surface, thus leading to a refractive index change. By this way, we can detect and recognize a certain $\mathrm{Ag}$ out of a large number of different protein molecules by the specific reaction of $A g-A b$ binding event.

\subsubsection{Basics of antigen-antibody interaction}

The antigens and the antibodies combine specifically with each other. This interaction is called Antigen-Antibody reaction and is usually abbreviated as $A g-A b$ reaction. An Ab will bind only strongly with the specific $A g$ which causes its production in a living organism. The specificity may be compared to a Lock and Key system [49]. The bonds between them are all noncovalent in nature. They include hydrogen bonds, electrostatic bonds (ionic bonds), Van der Waals forces and hydrophobic bonds, as illustrated in Fig. 3.6 [97]. Each individual bond is weak, but the combination of multiple bonds forms a strong interaction which ensures that an $\mathrm{Ag}$ is tightly bound to its corresponding $\mathrm{Ab}$. Since the $\mathrm{Ag}-\mathrm{Ab}$ interactions are non-covalent, they are by their nature reversible. In brief, an $\mathrm{Ag}$ specifically and reversibly binds with its homologous $\mathrm{Ab}$ via intermolecular forces. 


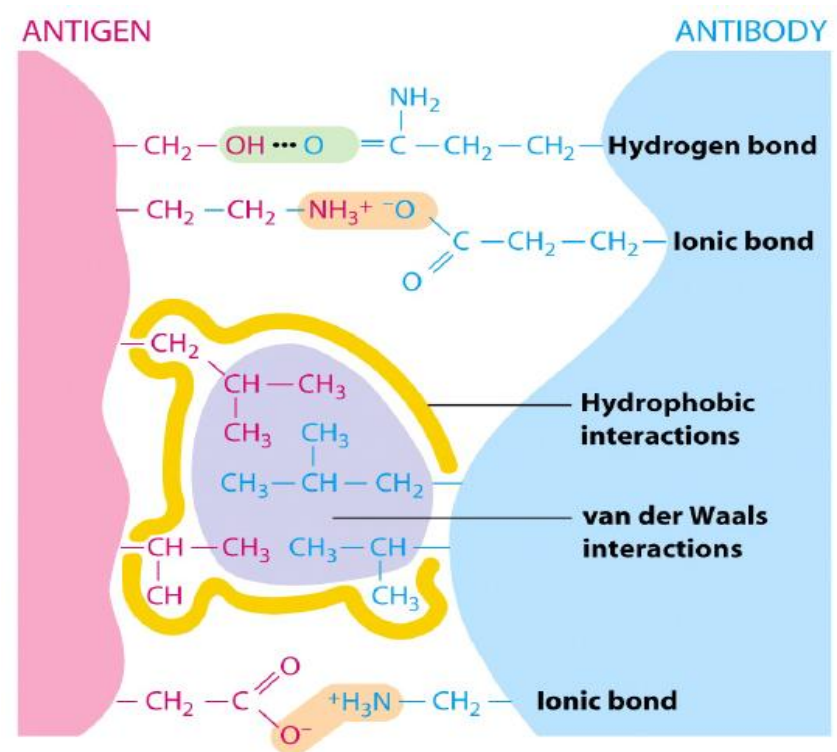

Fig. 3.6 Antigen-antibody (Ag-Ab) interactions: an Ag binds strongly to its homologous $A b$ and vice versa by non-covalent interactions, based on intermolecular forces, i.e., electrostatic (ionic) forces, hydrogen bonding, hydrophobic bonding and Van der Waals forces (courtesy from Ref. [97]).

\subsubsection{Immobilization of antibody on the $\mathrm{Si}_{3} \mathrm{~N}_{4}$ surface}

The $\mathrm{Si}_{3} \mathrm{~N}_{4}$ surface is known to be hydrated in an aqueous solution and silanol groups are generated at the surface [98-101]. The $\mathrm{Si}_{3} \mathrm{~N}_{4}$ surface can therefore be chemically modified with functional groups for immobilization of antibody molecules. We followed the standard immobilization process developed by IMEnz B.V. [102]. The process consists of 5 steps as shown in Fig.3.7:

* Cleaning and activation to generate a silanol surface $(-\mathrm{Si}-\mathrm{OH})$

- Silanization to generate an amino surface $\left(-\mathrm{NH}_{2}\right)$

* Glutaraldehyde treatment to generate an aldehyde surface $(-\mathrm{CH}=\mathrm{O})$

* Antibody immobilization

* Blocking of remaining aldehyde groups

The details of these steps are presented in Appendix A1. 

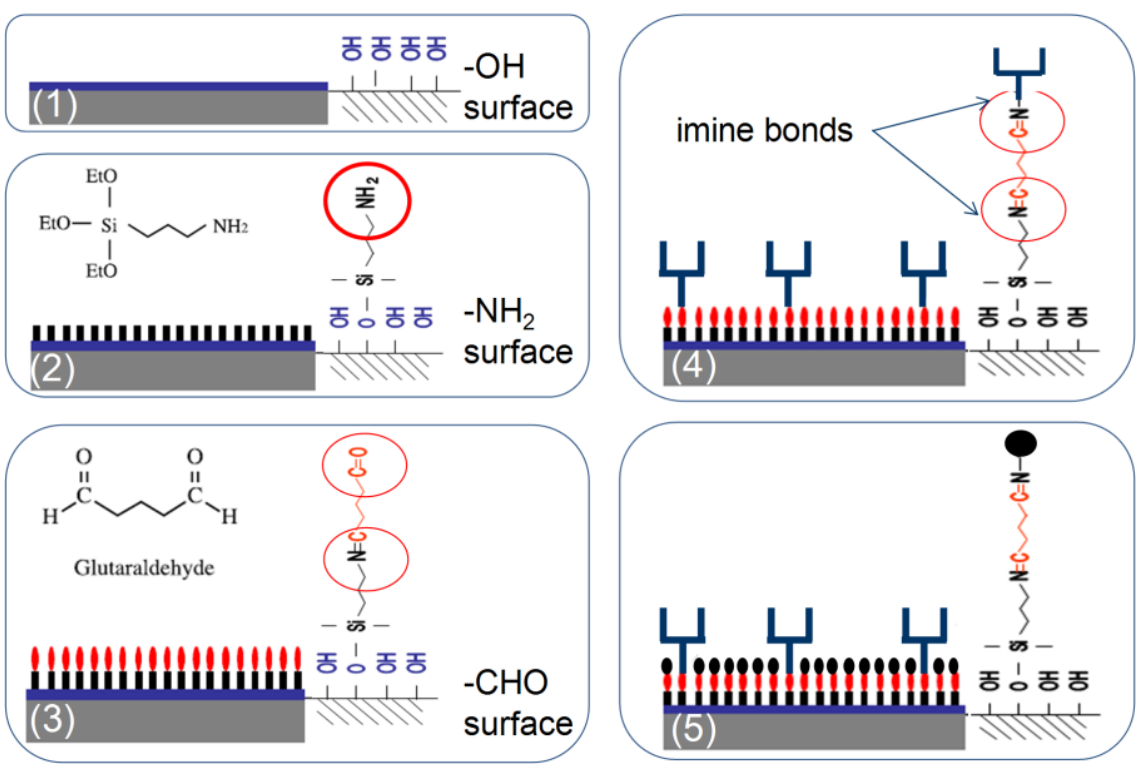

Fig. 3.7 Immobilization process of PepN-antibody on the $\mathrm{Si}_{3} \mathrm{~N}_{4}$ gratedwaveguide surface [102]: (1) cleaning and surface activation, (2) silanization, (3) glutaraldehyde treatment, (4) antibody immobilization, (5) Blocking of remaining aldehyde groups.

\subsubsection{PepN enzyme sensing: results and discussion}

In this section, we report on the performance of a GWG-based device in label-free surface bio-sensing (see Fig. 3.8). Direct sensing means that the analyte molecule is the target molecule and that it is the capture of the analyte molecules by receptor molecules incorporated in the thin adlayer which is monitored [19].

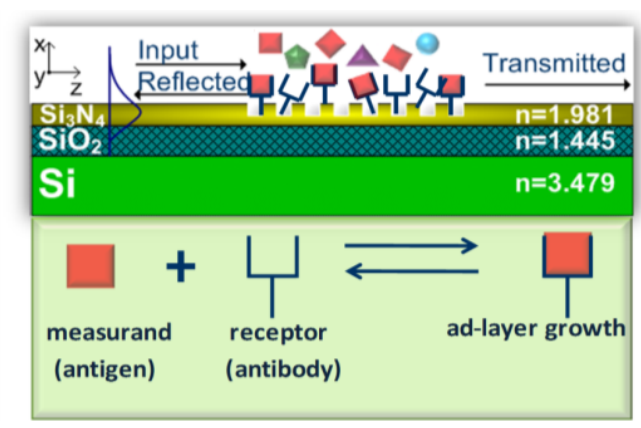

Fig. 3.8 Schematic 2D cross-section of the device for direct, label-free surface bio-sensing. 
As analyte we applied the PepN enzyme, a large molecule with a molecular weight of $M=95000$ [103]. A cell-free extract (CFE) solution, containing the target PepN enzyme and other proteins, was delivered by IMEnz B.V (see Section 3.5.3 above). The same company delivered as receptor molecule antibodies, specific for this enzyme, and a 5-step process for immobilizing these antibodies on the surface of the GWC device [104]. It was known that this PepN solution would saturate the receptors. We have not characterized the geometrical structure of the obtained adlayer, so we do not know whether the whole inner side of the grooves is covered. Uncovered $\mathrm{Si}_{3} \mathrm{~N}_{4}$ surfaces are not harmful, because we have observed that the solution on top of a grating without ad layer does not induce any spectral shift.

The resonant wavelengths of the GWG-based biosensor recorded after each of the five treatments (see Fig. 3.7) are plotted in Fig. 3.9.

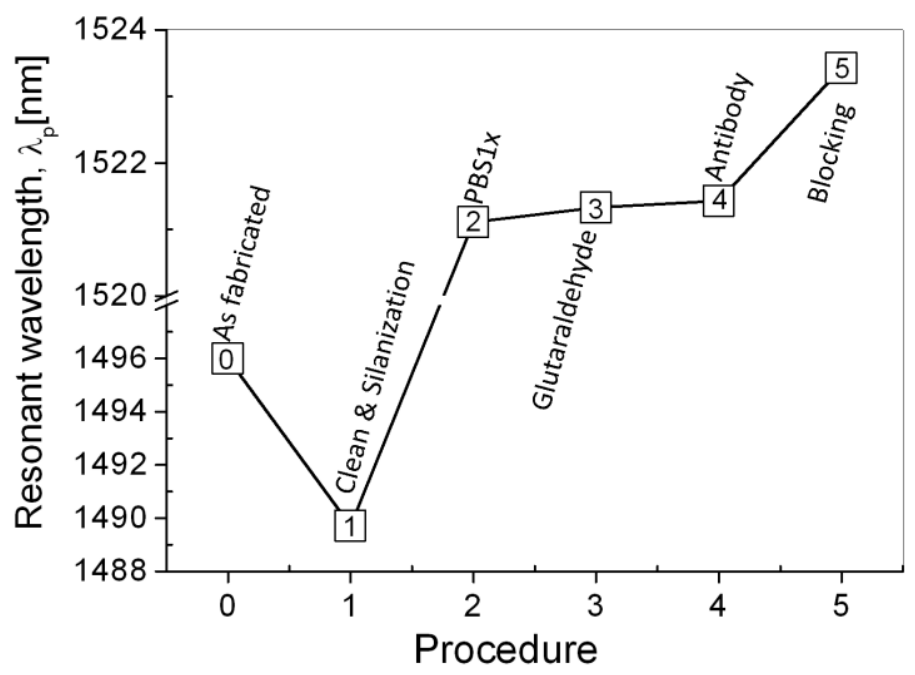

Fig. 3.9 Resonant wavelength of a GWG sensor after the different treatments: (O) as fabricated, (1) cleaning and surface activation - air top cladding; (2) silanization, (3) glutaraldehyde treatment, (4) antibody immobilization, (5) Blocking of remaining aldehyde groups - watery top cladding. The blue-shift from step 0 to step 1 is due to the thickness of $\mathrm{Si}_{3} \mathrm{~N}_{4}$ reduced after cleaning process, while red-shifts after each step of immobilization process are due to the increase in refractive index of the bulk or adlayer growth. The big jump from (1) to (2) is caused by applying a watery top cladding. 
Note that the resonant wavelength of the as-fabricated device is corresponding to the (sharpest) peak of the transmission spectrum shown in Fig. 3.2. After the cleaning process, the native oxide and a thin film of the $\mathrm{Si}_{3} \mathrm{~N}_{4}$ layer were removed, thus a blue-shift was observed, i.e., from step 0 (as-fabricated) to step 1 (cleaning and silanization). Then, the red-shifts after each step of the immobilization process were monitored. They are due to the increase in refractive index of the bulk solutions, which are solutions based on the PBS buffer. Note that each measurement was done at the first moment when the sample was applied. Therefore, the wavelength shifts were due to bulk index change only, not due to layer-growth at that moment.

Once the antibody was immobilized on the GWC surface and blocking, washing and drying steps were applied, the CFE solution was added to the cuvette. The antigen-antibody interaction, which results in an effective growth of the ad layer, was followed by optically monitoring the transmission spectra of the GWG.

Figure 3.10a shows the shift of the fringe spectra at different times (colored curves). High frequency noise was removed from the spectra using low-pass filtering in the Fourier domain (black curves). The time dependence of this shift is shown in Fig. 3.10b. Within 45 minutes the shift approached a constant value, indicating that the chemical equilibrium state (in this case saturation of the receptors) was reached. The shift of fringe position, $\Delta \lambda_{p}$, shows a good fit with an exponential function of time $t$ according to $\Delta \lambda_{p}(t)=C\left(1-e^{-t / t_{o}}\right)$, where $C=342 \mathrm{pm}$ and $t_{o}=770 \mathrm{~s}$. The process is fairly slow, which can be attributed to the large volume of analyte particles and/or the fact that they have to diffuse inside the grooves.

The final shift, $\Delta \lambda_{\text {ptotal }} \sim 342 \mathrm{pm}$, can be attributed to the growth of an adlayer with a thickness change of $\Delta h=1.9 \mathrm{~nm}$, a value which has been calculated using the grating mode solver (CMS) method, assuming that also the side walls of the grating grooves have been covered, and where the refractive index of PepN has been assumed to be $\sim 1.5$ (the value generally taken for proteins) [105]. Thus, $\partial \lambda_{p} / \partial h \approx 0.18$ and, using the earlier reported experimental value of $L O D_{\lambda}=0.8 \mathrm{pm}$, the resolution (LOD) for adlayer growth is $L O D_{h}=4.4 \mathrm{pm}$. 

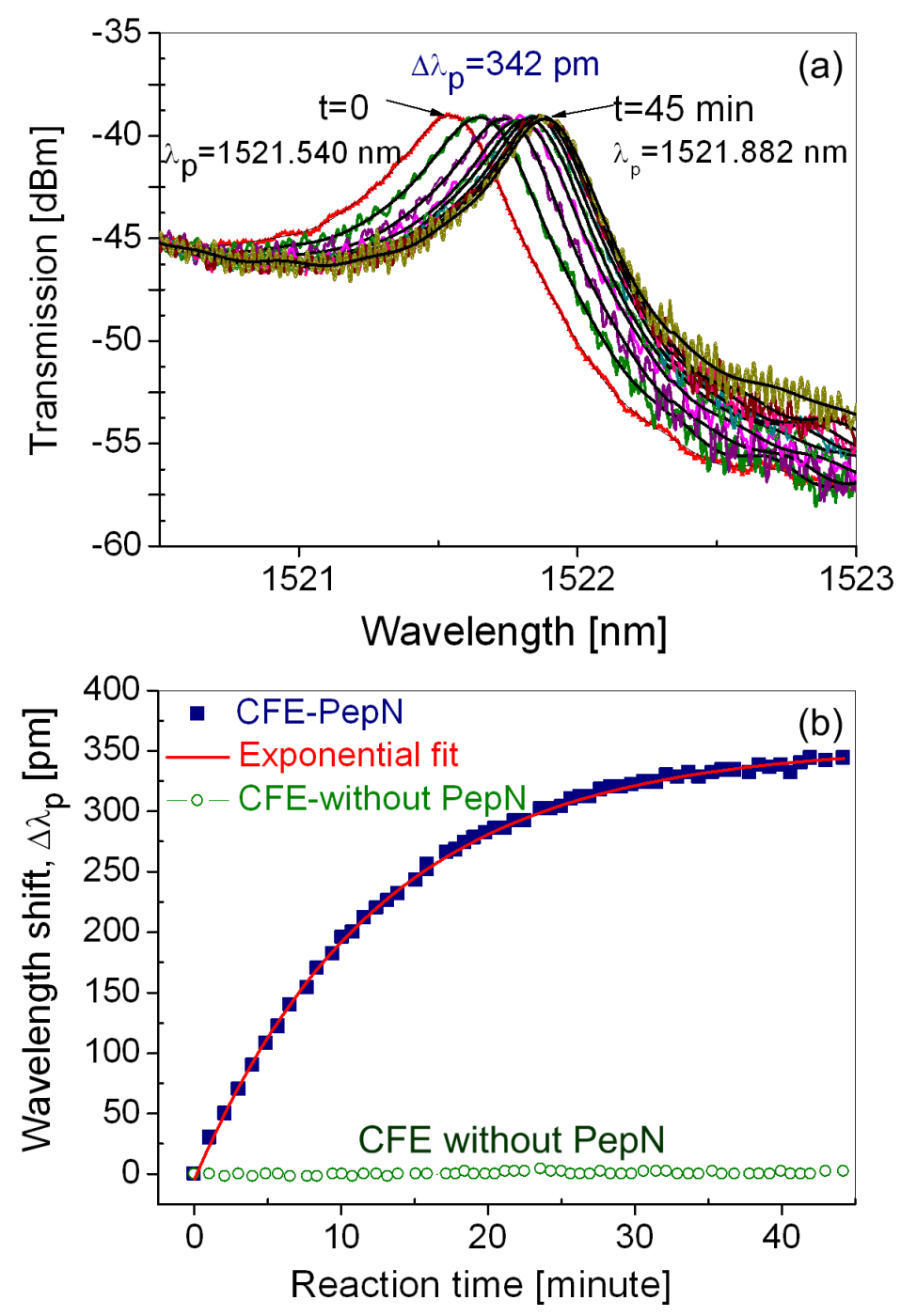

Fig. 3.10 (a) Transmission spectra of the label-free protein sensor, and (b) Peak-wavelength shift versus antigen-antibody interaction time for cell-free extracts (CFE) with and without PepN. The horizontal line of data points, labeled "CFE without PepN" indicates no reaction without corresponding antigen (i.e., PepN).

The experiments were also carried out with the same solution, but without PepN. No spectral shift was observed (circles in Fig. 3.10b), which shows that the adlayer growth due to the PepN solution is an effect of the PepN molecules only and that no nonspecific adsorption of the other proteins was interfering. 
Assuming the specific weight of PepN to be equal to that of water, i.e., $\rho=1 \mathrm{~g} / \mathrm{cm}^{3}$, the adlayer of thickness $(\Delta h=1.9 \mathrm{~nm})$ corresponds to an effective analyte surface density of $d=\rho \times \Delta h \times N_{A} / M=1.2 \times 10^{12} / \mathrm{cm}^{2}$ (with $N_{A}=6.02 \times 10^{23}$, the Avogadro's number and $M=95000$, the PepN molecular weight). It now follows for the limit of detection for surface density of $L o D_{d}: L o D_{d}=\left(L o D_{h} / \Delta h\right) \times d=3 \times 10^{9}$ PepN molecules per $\mathrm{cm}^{2}$.

\subsection{Conclusions}

We have evaluated the potential of a special integrated optical grating sensor for determining chemical concentrations. In contrary to many other grating sensors, this one relies on the measurand-induced shift of a special feature of the transmission spectrum. It has been shown that refractive indices of a solution above the grating can be determined with a resolution or LOD of 6 $\times 10^{-6}$ RIU. This affords to measure, e.g., the concentration of PBS, provided that it is the only solute in a watery solution, with a resolution of 0.005 weight percent. Furthermore, direct label-free sensing of a large protein molecule, such as the PepN enzyme $(M=95000)$, has been demonstrated. By immobilizing on top of the grating a thin layer which contains antibodies as receptors specific for PepN, effective thickness changes of that layer with a resolution or LOD of $4 \mathrm{pm}$ could be monitored. For PepN this is equivalent to the capture of $3 \times 10^{9}$ molecules per $\mathrm{cm}^{2}$.

The resolution (or LOD) can be especially improved by the use of peripheral equipment of higher quality: a tunable laser with repeatability of the wavelength better than $0.3 \mathrm{pm}$ and a temperature stabilization better than the current $\pm 0.03 \mathrm{~K}$.

The sensor structure is simple and can be realized by well controlled microtechnologies which are compatible with CMOS and lab-on-a-chip technologies.

A strong point is the small size: the grating section has an area of about $120 \times 15 \mu^{2}$, the complete sensor including the light transport channels could be as small as $\sim 200 \times 15 \mu \mathrm{m}^{2}$. This small size is very relevant for the realization of sensor arrays or for device integration with lab-on-a-chip systems. 



\section{Mechano-optical read-out system for hydrogen sensor}

In this chapter, we present the preliminary design, fabrication and characterization of a mechano-optical read-out system based on a cantilever that is suspended above a grated waveguide. The design parameters required to obtain a functioning device are considered. In particular, care is taken to prevent cantilever stiction and to minimize (unwanted) initial bending. Devices that integrate a cantilever and a grated waveguide have been fabricated successfully using microelectromechanical systems (MEMS) techniques. Several technical problems encountered during the preparation of such integrated devices (i.e., grating production, surface roughness, facet quality) will be discussed, and solutions to address these issues will be given. More details of some fabrication processes are given in Appendices. Preliminarily experimental results, showing the sensing of cantilever nanodisplacements, and so the feasibility of proposed readout principle, are presented. 


\subsection{Introduction and outline}

Cantilever-based hydrogen sensors, consisting of cantilevers coated with a hydrogen-absorbing palladium (Pd) layer, have attracted the attention of many researchers $[43,44,54,55,106-108]$. In such devices, the surface of the Pd film catalyzes the breakdown of molecular hydrogen $\left(\mathrm{H}_{2}\right)$ into atomic hydrogen $([\mathrm{H}])$, which can be reversibly absorbed into the Pd film volume [106]. The absorption-induced stress leads to hydrogen-concentrationdependent cantilever bending, which can be determined by means of optical beam deflection [107, 108], capacitance- [54], or piezo-resistance- [55] based read-out.

We propose a novel, compact and potentially highly sensitive read-out method comprising a cantilever suspended above a grated waveguide (GWG) for hydrogen sensing (see Fig. 4.1) [60, 109]. Such a device enables the detection of concentration changes of hydrogen through nanodisplacements of the cantilever, which is monitored optically by shifts of the resonance peaks of the transmission spectrum.

This chapter is organized as follows. Section 4.2 presents the device structure and expressions for the sensitivity. Gas absorption-induced cantilever bending and cantilever design considerations are discussed in sections 4.3 and 4.4, respectively. Descriptions of the fabrication and characterization of the device are presented in Section 4.5: sub-section 4.5.1 describes the fabrication process, developed for the functional optimization of the integrated chip, and in sub-section 4.5.2 we discuss fabrication issues and characterization results which include the cantilever-deflectiondependent spectral shift of the transmission. The chapter ends with conclusions (section 4.6).

\subsection{Device structure and sensitivity}

A schematic of the GWG-cantilever set-up is shown in Fig. 4.1. The GWG-cantilever integrated system used as a mechano-optical sensor utilizes the transduction chain:

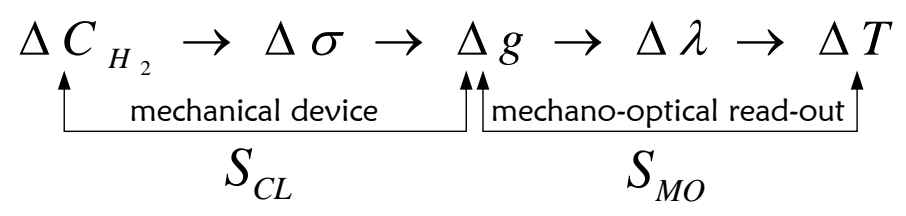


where $\Delta X$ denotes a change of the parameter $X$, with $C_{H_{2}}$ the concentration of hydrogen gas, $\sigma$ the hydrogen absorption-induced stress, $g$ the gap between the GWG and cantilever (CL), $\lambda$ the wavelength, and $T$ the transmittance.

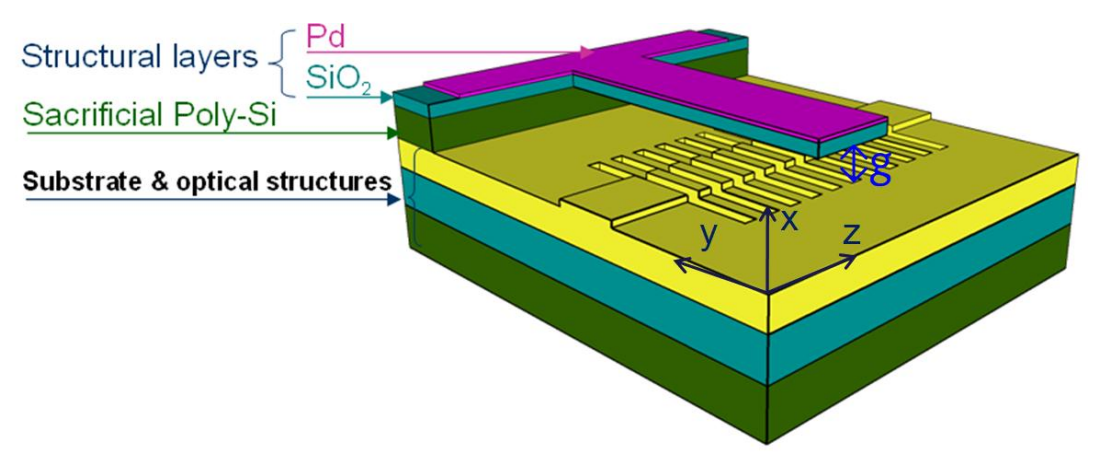

Fig. 4.1 Schematic of the GWG-CL set-up

As introduced in Chapter 2 (Eq. 2.6), the (wavelength dependent) sensitivity of the gas sensor, $S_{\text {gas }}$, is given by

$$
S_{g a s} \equiv\left|\left(\frac{1}{T} \frac{\partial T}{\partial C}\right)_{\lambda}\right|=\left|\left(\frac{\partial \ln T}{\partial C}\right)_{\lambda}\right|=\underbrace{\left|\left(\frac{\partial \ln T}{\partial g}\right)_{\lambda}\right|}_{\equiv S_{M O}} \underbrace{\left(\frac{\partial g}{\partial C}\right) \mid}_{\equiv S_{C L}}
$$

The sensitivity $S_{g a s}$ can be divided into two parts:

* the sensitivity of the mechanical device $S_{C L}$, which indicates how sensitive the integrated cantilever detects the analyte (hydrogen gas), and

* the sensitivity of the mechano-optical read-out $S_{M O}$, which reflects the conversion of the stress-induce bending of the integrated cantilever into change in optical signal of the GWG device.

In order to achieve a high sensitivity of the sensor (high $S_{g a s}$ ), the partial sensitivities $S_{M O}$ and $S_{C L}$ need to be optimized. The quantity $S_{M O}$ depends on structural parameters of the GWC and the initial gap between GWG and $\mathrm{CL}$. Design parameters of the GWG structure (for the aim of obtaining a functioning device) remain the same as the ones used for bulk concentration and biosensors, described in Chapter 3. The optimization of the mechano- 
optical read-out, i.e., the optimization of $S_{M O}$, will be discussed in Chapter 6 (Section 6.3). Whereas the quantity $S_{C L}$ depends on $\mathrm{CL}$ geometry and its material properties, which include mechanical properties and properties related to gas absorption. In this chapter, design parameters of the cantilever are in particular discussed considering the sensitivity $S_{C L}$ and the fabrication technology, enabling the integration of the $\mathrm{CL}$ suspended above the GWG.

\subsection{Gas absorption induced cantilever bending}

The sensitivity of the mechanical structure in the GWG-CL integrated system defined by (see more: sensitivity definition for full GWG-CL system in Chapter 6, Section 6.3)

$$
S_{C L} \equiv\left|\frac{\partial g}{\partial C}\right|
$$

where $g$ is the GWG-CL gap and $C$ is the gas concentration. According to Refs. [43, 110], the hydrogen absorption induced cantilever displacement, $\Delta g$, is expressed as

$$
\Delta g=3\left(\alpha_{1}-\alpha_{2}\right)\left(\frac{t_{1}+t_{2}}{K t_{2}^{2}}\right) C_{H} L_{C L}^{2},
$$

where $L_{C L}$ is the cantilever length, $C_{H}$ is the gas concentration in $\mathrm{Pd}$, $t_{i=1,2}$ and $\alpha_{i=1,2}$ are the layer thicknesses and concentration related expansion coefficients with the subscripts referring to the layers of the bimaterial cantilever, respectively. The quantity $K$ is given by

$$
K=4+6\left(\frac{t_{1}}{t_{2}}\right)+4\left(\frac{t_{1}}{t_{2}}\right)^{2}+\left(\frac{E_{1}}{E_{2}}\right)\left(\frac{t_{1}}{t_{2}}\right)^{3}+\left(\frac{E_{2}}{E_{1}}\right)\left(\frac{t_{2}}{t_{1}}\right)
$$

with $E_{i=1,2}$ the Young's modulus of the two materials, which are assumed not to change during the gas-absorption.

Equations (Eq. 4.3) and (Eq. 4.4) show that for any given gas concentration $C_{H}$, the absorption-induced deflection $\Delta g$ of the $\mathrm{CL}$ varies as a function of the length, thicknesses and material properties of the bimaterial $\mathrm{CL}$, and that it can be optimized for maximum response. One material in a bilayer $\mathrm{CL}$ can be chosen to be noninteracting with the analyte. For instance, 
in this thesis, the bimaterial cantilever is made of silicon oxide (not interacting with $\mathrm{H}_{2}$ ) and coated with a palladium (Pd) receptor layer (absorbing $\mathrm{H}_{2}$ ). Absorption of $\mathrm{H}_{2}$ into the Pd film results in the formation of $\alpha$-phase (hydrogen-poor) and $\beta$-phase (hydrogen-rich) palladium-hydride [111], and can cause volume expansions of up to $10 \%$ [43] or $20 \%$ [56]. According to Sievert's law, the gas concentration $C$ is directly proportional to the square root of the external pressure $p_{H_{2}}$ (and thus the concentration $C_{\mathrm{H}_{2}}$ ) of molecular hydrogen, i.e., $C_{H} \propto \sqrt{p_{H_{2}}} \propto \sqrt{C_{H_{2}}}[43,56,58]$. A highly sensitive device, i.e., high $S_{C L}$, can detect a very small amount of $C_{H}$ by inducing a large $\Delta g$. To obtain a high $S_{C L}$, all relevant structural parameters listed in Table 4.1 need to be investigated and optimized. Focused on a functioning device and based on Eq. 4.3, the blue boxed parameters can firstly be chosen, while the yellow boxed parameters require further consideration as presented in the next section (section 4.4).

Table 4.1 Design parameters that need to be optimized to obtain a high $S_{C L}$, the blue boxed parameters can firstly be chosen, while the yellow boxed parameters require further consideration as presented in section 4.4. The coordinate system is given in Fig. 4.1.

\begin{tabular}{lll}
\hline Design parameters & $\mathrm{Pd}$ & $\mathrm{SiO}_{2}$ \\
\hline Length (along y) & $L_{1}$ & $L_{2}=L_{C L}$ \\
Width (along z) & $W_{1}$ & $W_{2}$ \\
Thickness (along $\mathrm{x}$ ) & $t_{1}$ & $t_{2}$ \\
\hline Initial gap between GWG and cantilever & $g_{0}$ & \\
\hline
\end{tabular}

\subsection{Cantilever design consideration}

\subsubsection{Residual stress-induced initial bending}

In practice, thin films can not be deposited stress free, owing to stress developed during cooling down after processing, which is so-called residual stress. The difference between residual stresses in the two materials of the bilayer cantilever results in an initial bending of the bilayer cantilever. Initial bending may lead to either stiction (CL-CWW) or upbending such that the $\mathrm{CL}$ 
will no longer interact with GWG modes, which both would frustrate proper operation. This initial bending $\delta_{o}$ is expressed by [112-118]

$$
\delta_{0}=L_{C L}^{2} \frac{3 w_{1} w_{2} E_{1} E_{2} t_{1} t_{2}\left(t_{1}+t_{2}\right)\left(\frac{\sigma_{01}}{E_{1}}-\frac{\sigma_{02}}{E_{2}}\right)\left(\alpha_{\tau_{1}}-\alpha_{\tau_{2}}\right) \Delta \tau}{\left(w_{1} E_{1} t_{1}^{2}\right)^{2}+\left(w_{2} E_{2} t_{2}^{2}\right)^{2}+2 w_{1} w_{2} E_{1} E_{2} t_{1} t_{2}\left(2 t_{1}^{2}+3 t_{1} t_{2}+2 t_{2}^{2}\right)},
$$

where $\delta_{0}, L_{C L}, w_{i}$, and $t_{i}$ are the initial bending, length, width, and thickness of the $\mathrm{CL} ; E_{i}, \sigma_{0 i}$, and $\alpha_{\tau_{i}}$ are Young's modulus, the residual stress and thermal expansion coefficient of material $i$, with the subscripts $i=1$ and 2 representing the $\mathrm{Pd}$ and $\mathrm{SiO}_{2}$ layers, respectively. The residual stresses of the films were determined based on Stoney's formula

$$
\sigma_{0 i}=\frac{E_{s} t_{i}^{2}}{6 R\left(1-v_{s}\right)}
$$

where $E_{s}$ and $v_{s}$ are Young's modulus (or stiffness) and the Poisson ratio of the substrate, and $R$ is the radius of curvature, obtained by measuring the wafer curvature, $1 / R$, due to deposition of the film on a silicon substrate [1]; their values are $\sigma_{01}=1000 \mathrm{MPa}$ (tensile stress) and $\sigma_{02} \sim-310 \mathrm{MPa}$ (compressive stress) for $\mathrm{Pd}$ and $\mathrm{SiO}_{2}$ films, respectively.

Equation 4.5 shows that the initial bending is equal zero $\left(\delta_{0}=0\right)$ if the thickness of the receptor layer is zero $\left(t_{1}=0\right.$, the single-material cantilever), or if the residual stress-stiffness ratio satisfies the condition of $\sigma_{01} / E_{1}=\sigma_{02} / E_{2}$. None of these is the case for the $\mathrm{Pd} / \mathrm{SiO}_{2}$ bimaterial cantilever. Depending on the status of residual stress in each material and the difference between two ratios $\sigma_{01} / E_{1}$ and $\sigma_{02} / E_{2}$, the bilayer $\mathrm{CL}$ may bend upwards or downwards after release (see Fig. 4.2). 
(a)
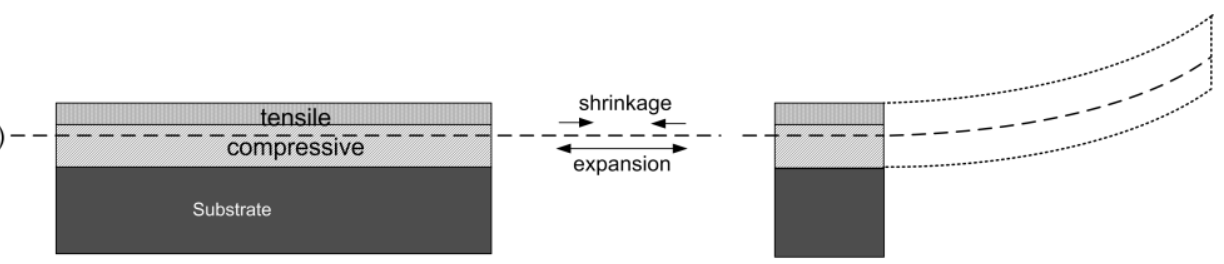

(b)

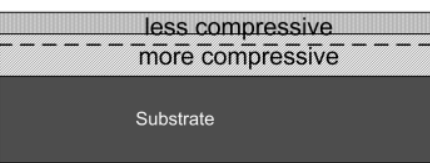

smaller expansion bigger expansion

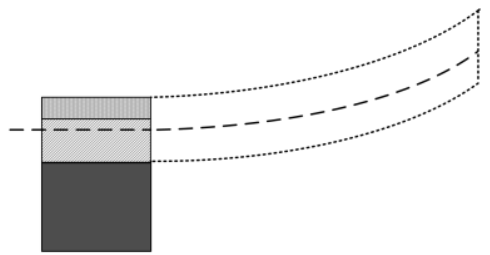

(c)

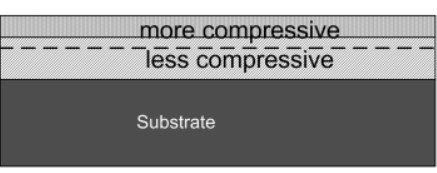

bigger expansion smaller expansion

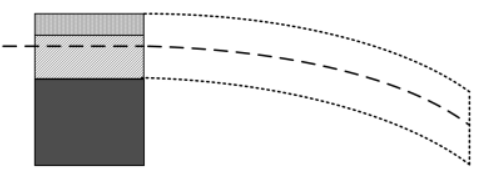

(d)
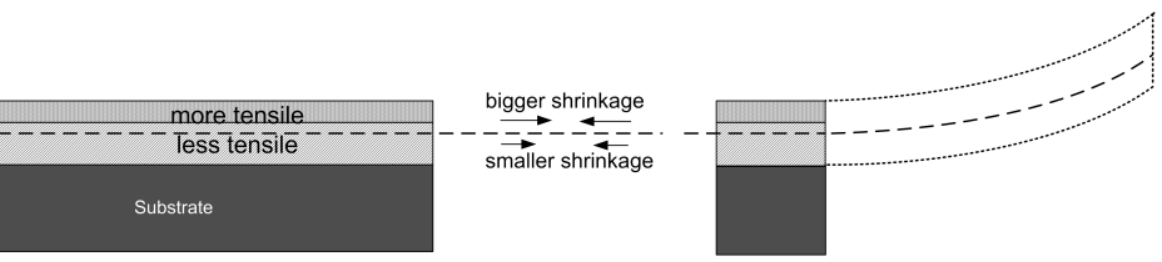

(e)

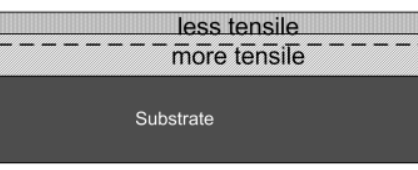

smaller shrinkage
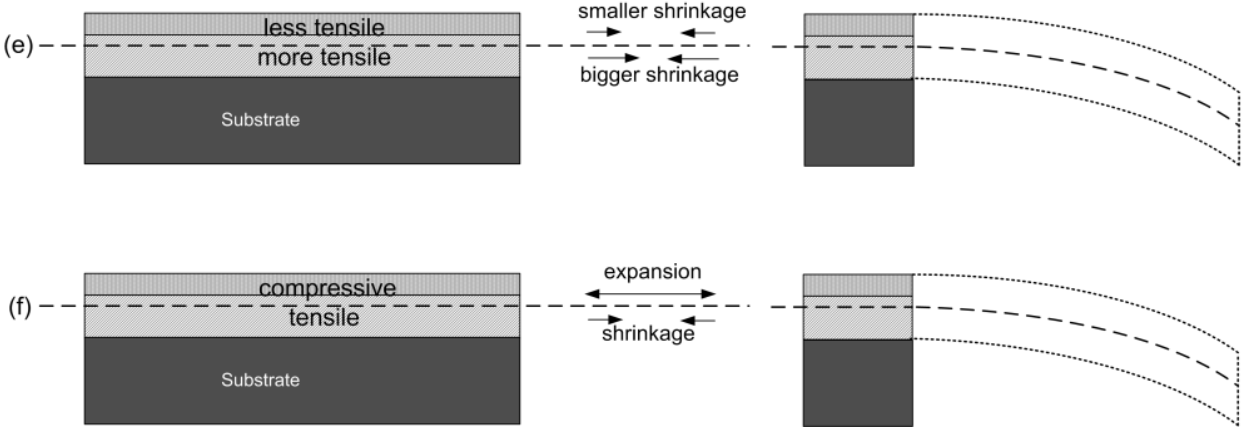

Fig. 4.2 Behavior of a bimaterial cantilever after release. Depending on the status of residual stress in each material and the difference between two ratios $\sigma_{01} / E_{1}$ and $\sigma_{02} / E_{2}$, the bilayer cantilever may bend upwards or downwards after release. Initial bending of the bilayer CL presented here $\left(\mathrm{Pd} / \mathrm{SiO}_{2}\right)$ is similar to the case (a), while its absorption induced bending is similar to the case (f). 
Since residual stress and stiffness $\left(E_{i}\right)$ of $\mathrm{Pd}$ and $\mathrm{SiO}_{2}$ are different, one method to minimize initial bending of the $\mathrm{Pd} / \mathrm{SiO}_{2}$ bimaterial $\mathrm{CL}$ is to control the length of the cantilever and the thickness of both $\mathrm{Pd}$ and $\mathrm{SiO}_{2}$ layers. Another option is to modify the configuration of the mechanical system. Three solutions that can be proposed are:

* Varying the structural parameters of the Pd layer or making holes in the bilayer structure to reduce residual stress.

* Making a doubly-clamped bimaterial cantilever (bridge) instead of a singly-clamped cantilever.

* Incorporating other layers in the main bilayer structure, for instance, making the $\mathrm{Pd} / \mathrm{Si}_{3} \mathrm{~N}_{4} / \mathrm{SiO}_{2} / \mathrm{Si}_{3} \mathrm{~N}_{4}$ multi-material cantilever. In this way, thin film stresses compensate one another, and initial bending of the cantilever is reduced significantly [119].

It's necessary to numerically simulate the above proposed structures to predict their behavior prior to fabrication. The INTELLISUITE software package, which is a finite-element-based mechanical modelling package, will be used to perform the simulations as presented in Chapter 5. This thesis focuses on a proof of concept, not on fully optimized devices. The possibility of low initial bending is presented in Chapter 5. It is also noticed that a reduction of initial bending simultaneously decreases the sensitivity of the cantilever to gas absorption-induced deflection as both initial bending and bending via the absorption are based on same mechanism (see Section 4.3).

\subsubsection{Stiction}

In the fabrication process of the GWG-CL device (as described in Section 0 below), the cantilever is released by removing the sacrificial silicon layer underneath it. If wet etching process is used, stiction issue needs to be taken into account [120-125]. Stiction of the cantilever on the GWG surface, caused by adhesive forces, i.e., capillary forces, hydrogen bridging, van de Waals forces, will limit the cantilever length, which is given by equation 11 of Ref. [121]:

$$
L_{C L}<L_{\text {critical }}=\sqrt[4]{\frac{3}{8} \frac{E_{2} t_{2}^{3} g_{0}^{2}}{\gamma_{s}}},
$$


with $\gamma_{s}$ the adhesion energy per unit area. If the cantilever length exceeds the critical length, i.e., $L_{C L}>L_{\text {critical }}$, stiction will occur. Assuming an adhesion energy of water $\gamma_{s}=100 \mathrm{~mJ} / \mathrm{m}^{2}$ [121], and Young's modulus of $\mathrm{SiO}_{2}$ $E_{2}=61 \mathrm{GPa}$ [126], the critical length of the cantilever can be evaluated as shown in Fig. 4.3. If for example the initial gap is given by $g_{0}=400 \mathrm{~nm}$ and the thickness of cantilever is $800 \mathrm{~nm}$, the critical length is $L_{\text {critical }} \sim 12 \mu \mathrm{m}$. Note that in Eq. 4.7, only the $\mathrm{SiO}_{2}$ layer is considered and the effect of the relatively thin Pd layer can be neglected.

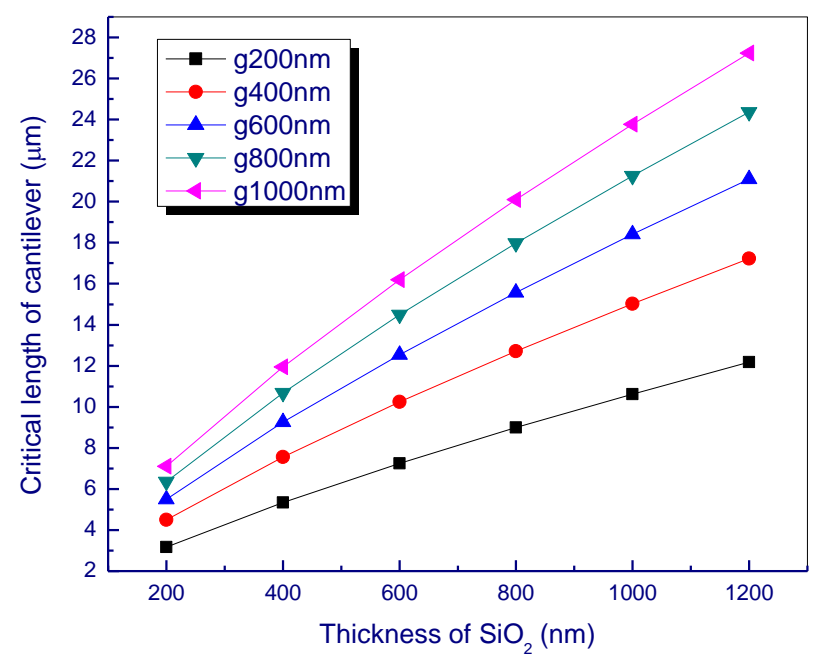

Fig. 4.3 Critical length of the cantilever as function of its thickness at different gaps, assuming an adhesion energy of water $\gamma_{s}=100 \mathrm{~mJ} / \mathrm{m}^{2}$ and Young's modulus $E_{2}=61 \mathrm{GPa}$.

To avoid the stiction problem after the wet etching process, special drying methods, like supercritical drying and freeze drying, can be used [121, 125, 127]. At MESA+, the freeze-drying process is used. Details of the method are given in Appendix A2 [128]. In addition, surface roughness of the grating, which reduces the contact area between $\mathrm{CL}$ and $\mathrm{GWG}$ may prevent stiction. Therefore, a longer cantilever length than the aforementioned critical length can be chosen. Using dry etching can eliminate the stiction problem since no liquid is involved. However, also the etching selectivity for the involved materials is a major aspect in choosing between dry etching and wet etching. 


\subsubsection{Length of Pd layer}

The big advantage of the CL-based approach is that the light itself does not need to directly interact with the (absorbing) Pd layer. Sensing is based on the effect of changes in the $C L$ position, owing to the interaction between the integrated $C L$ suspended above the CWG and the evanescent tail of the GWG modal field. Fig. 4.4 shows the optical loss, simulated using a mode solver [98], as a function of the length of the Pd layer (thickness $\left.t_{1}=50 \mathrm{~nm}[43,57]\right)$ for different $\mathrm{SiO}_{2}$ thicknesses. The initial gap $g_{0}=400 \mathrm{~nm}$ was chosen equal to the decay length of the evanescent field (i.e., 1/e of the evanescent peak value). If the cantilever is fully covered by $\mathrm{Pd}$, optical loss occurs, which increases if the thickness of $\mathrm{SiO}_{2}$ decreases. To avoid such unwanted absorption or scattering loss the $\mathrm{CL}$ is not covered in the region that overlaps the modal field. The length of the uncovered part of the $\mathrm{CL}$ is about $15 \mu \mathrm{m}$.
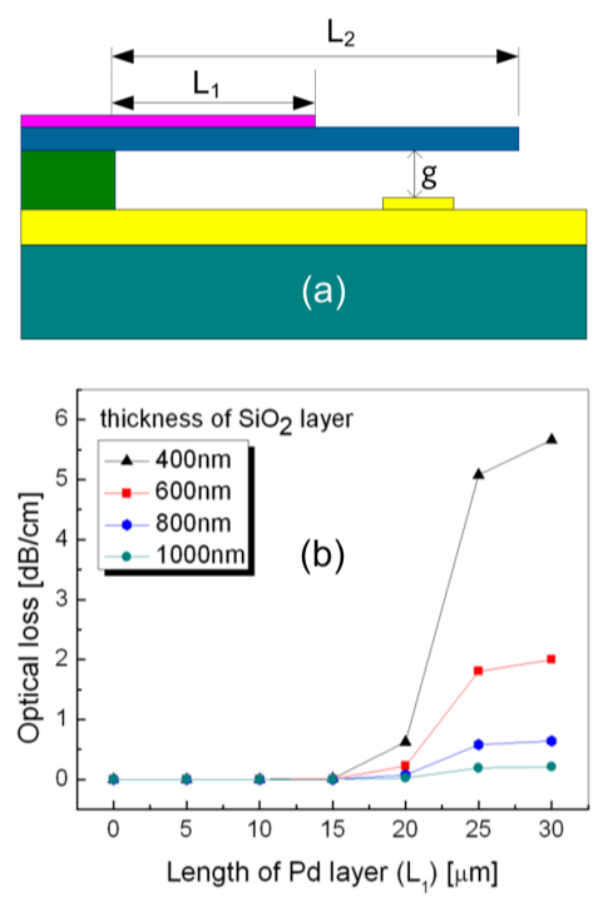

Fig. 4.4 (a) Schematic $2 D$ cross-section of the GWG-CL device; the layer materials are identified in Fig. 4.1. (b) Optical loss as a function of the length of the Pd layer for different $\mathrm{SiO}_{2}$ thicknesses, simulated using a mode solver. To avoid unwanted absorption or scattering loss the cantilever is not covered in the region that overlaps the modal field. 


\subsubsection{Preliminary design parameters}

Taking into account all aspects mentioned in sections 4.4.1 - 4.4.3, design parameters of the mechanical structure are preliminarily chosen as listed in the Table 4.2. The choice is a compromise between the given aspects, not a full optimization yet. For instance, the widths $w_{1}$ and $w_{2}$, which are not very critical for obtaining a functioning device and a high $S_{C L}$, can be chosen to be $w_{1}=w_{2}=20 \mu \mathrm{m}$. Note that in realization $w_{1}$ can be narrower than $w_{2}$, i.e., $w_{1}=w_{2}-2 \times 4 \mu \mathrm{m}$ (see Section 4.5 ), for the ease of alignment. The length of cantilever should be short enough to avoid stiction while remaining long enough to give sufficient room for the Pd layer and for the uncovered part $(\geq 15 \mu \mathrm{m})$. Therefore the length of the $\mathrm{SiO}_{2}$ cantilever is chosen to be $L_{C L}=L_{2}=30 \mu \mathrm{m}$ and the length of the Pd layer is varied from 5 to $15 \mu \mathrm{m}$. Similarly as for $L_{2}, t_{2}=800 \mathrm{~nm}$ is chosen. Other chosen parameters are $t_{1}=50 \mathrm{~nm}$ and $g_{o}=300 \mathrm{~nm}$.

Table 4.2 Preliminary design parameters used for fabrication, which are not optimized yet.

\begin{tabular}{lll}
\hline Preliminary design parameters & $\mathrm{Pd}$ & $\mathrm{SiO}_{2}$ \\
\hline Length $[\mu \mathrm{m}]$ & $L_{1}=5,10,15$ & $L_{2}=L_{C L}=30$ \\
Width $[\mu \mathrm{m}]$ & $W_{1}=20$ & $W_{2}=20$ \\
Thickness $[\mathrm{nm}]$ & $t_{1}=50$ & $t_{2}=800$ \\
\hline
\end{tabular}

Initial gap between CWG and cantilever [nm] $g_{o}=300$

\subsection{Fabrication and characterization of the mechano-optical read-out}

In this section, results related to the fabrication of a novel mechano-optical read-out of cantilever bending are presented. Integrated microcantileverGWG devices have been fabricated successfully using MEMS techniques. Several technical problems encountered during the preparation of such integrated devices (i.e., grating production, surface roughness, facet quality) will be discussed and solutions to address these issues will be given as well. 


\subsubsection{Fabrication flow chart}

The process flow chart of the fabrication of the device is shown in Fig. 4.5. An 8- $\mu \mathrm{m}$ thick $\mathrm{SiO}_{2}$ buffer layer was grown on a (100) Si wafer, using thermal oxidation. Next, a 275-nm thick $\mathrm{Si}_{3} \mathrm{~N}_{4}$ core layer was deposited using the stoichiometric low pressure chemical vapor deposition (LPCVD) technique. The refractive indices of $\mathrm{Si}_{3} \mathrm{~N}_{4}$ and $\mathrm{SiO}_{2}$ are 1.981 and 1.445 at the wavelength of $1.5 \mu \mathrm{m}$, respectively. A $5-\mu \mathrm{m}$ wide ridge waveguide, with 5 $\mathrm{nm}$ ridge height, was defined using photolithography, and etched into the $\mathrm{Si}_{3} \mathrm{~N}_{4}$ layer using the buffered hydrofluoric (BHF) wet etching process. The $490 \mathrm{~nm}$-period gratings, with a groove depth of $55 \mathrm{~nm}$ and a duty cycle near $50 \%$, were defined with laser interference lithography (LIL), using a Lloyd'smirror-setup, producing a pattern size $2.7 \times 10 \mathrm{~cm}^{2}$. Details of the LIL setup can be found in Ref. $[129,130]$. For our application, small alignment marks were added at strategic positions to enable proper alignment of gratings and cantilevers. A photolithographic mask was used to define the length (being $122.5 \mu \mathrm{m}$ ) and position of the gratings. The grating patterns were transferred into the $\mathrm{Si}_{3} \mathrm{~N}_{4}$ layer using reactive ion etching (RIE).

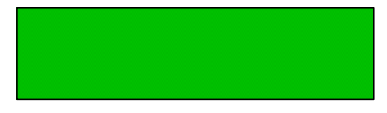

(1) Si wafer (100)

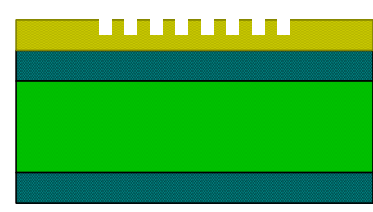

(4) Waveguide and Gratings

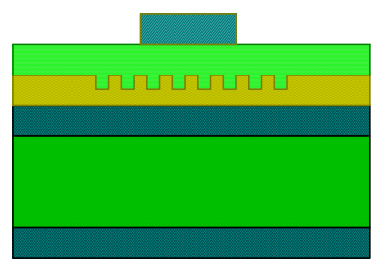

(7) Cantilever defined, RIE

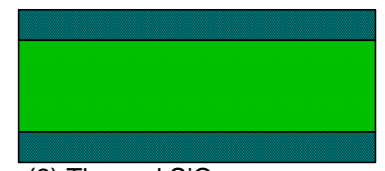

(2) Thermal $\mathrm{SiO}_{2}$

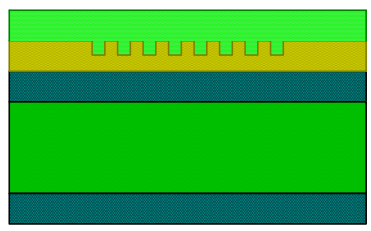

(5) LPCVD poly-Si

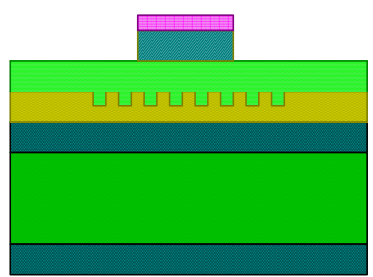

(8) Sputtering $\mathrm{Cr} / \mathrm{Pd}$ \& Lift-off

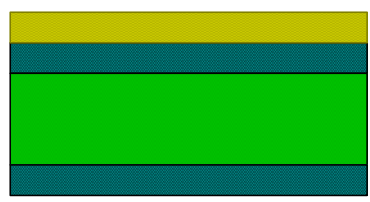

(3) LPCVD $\mathrm{Si}_{3} \mathrm{~N}_{4}$

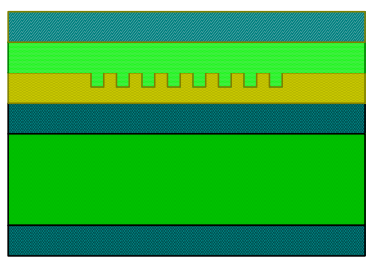

(6) TEOS SiO2

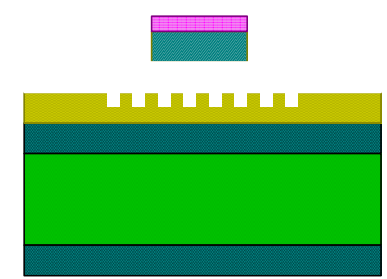

(9) poly-Si etched \& freeze-drying

Fig. 4.5 Process flow chart of fabrication of an integrated mechano-optical sensing device. 
In a next step, $300 \mathrm{~nm}$ thick sacrificial poly-Si and $800 \mathrm{~nm}$ thick tetraethyl-ortho-silicate (TEOS) $\mathrm{SiO}_{2}$ layers were deposited using LPCVD. Microcantilever patterns, with a length of $30 \mu \mathrm{m}$ and a width of $20 \mu \mathrm{m}$, were defined in photo-resist on the TEOS $\mathrm{SiO}_{2}$ layer by conventional photolithography, followed by removing unnecessary $\mathrm{SiO}_{2}$ areas using RIE. Then, metallic layers, viz. a $10 \mathrm{~nm} \mathrm{Cr}$ adhesion layer and a $50 \mathrm{~nm} \mathrm{Pd}$ receptor layer, were sputter-deposited at room temperature, and patterned using a lift-off process. Finally, microcantilevers were released by locally removing the sacrificial poly-Si layer, using one of two methods: (1) wet etching using a $5 \%$ tetramethylammonium hydroxide (TMAH) solution at $70^{\circ} \mathrm{C}$, followed by a freeze-drying process, or (2) $\mathrm{SF}_{6}$ reactive ion plasma dry etching.

\subsubsection{Fabrication issues and device characterization}

Several processing steps needed to be investigated and optimized in order to obtain well-performing devices with, e.g., low initial cantilever bending and uniform gratings.
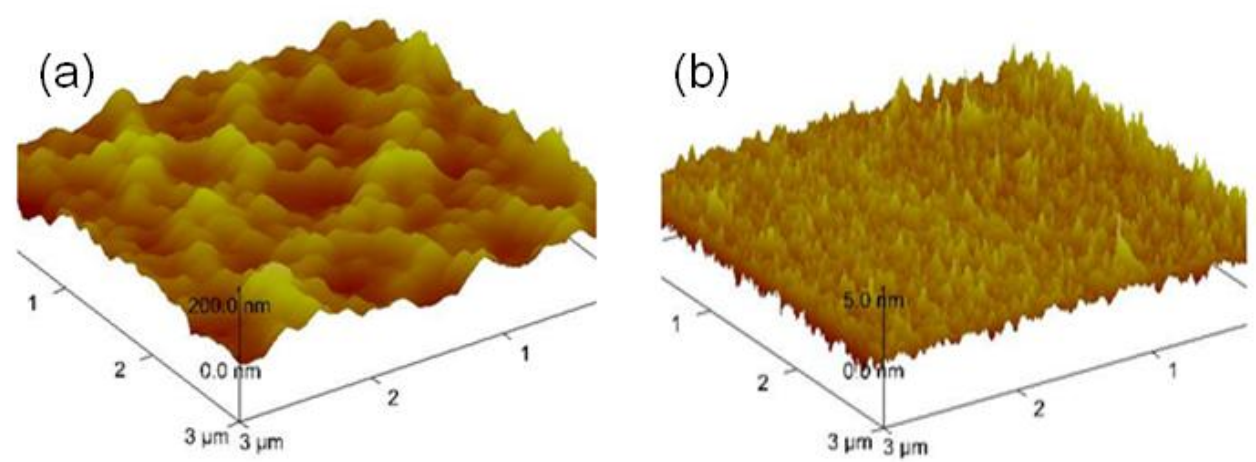

Fig. 4.6. Atomic force microscopy (AFM) topographic images of $\mathrm{Si}_{3} \mathrm{~N}_{4}$ surface roughness, after (a) $S F_{6}$ dry-etching $\left(R_{q}=25.6 \mathrm{~nm}\right)$ and (b) $T M A H$ wet etching $\left(R_{q}=0.46 \mathrm{~nm}\right)$.

The two possible methods for selective removal of the sacrificial poly-Si layer, as mentioned in the preceding subsection, have been experimentally investigated. An extra mask is required to protect un-etched regions when $\mathrm{SF}_{6}$ plasma dry-etching is used (see Appendix A3). In particular the impact of etching on the waveguiding material (i.e., $\mathrm{Si}_{3} \mathrm{~N}_{4}$ underneath the poly-Si) was investigated using atomic force microscopy (AFM) topographic surface measurements of the $\mathrm{Si}_{3} \mathrm{~N}_{4}$ layer. Fig. 4.6 shows the surface roughness of the 
$\mathrm{Si}_{3} \mathrm{~N}_{4}$ films caused by the dry-etching and wet-etching processes. The RMS roughness values $\left(R_{q}\right)$ in these cases are $25.6 \mathrm{~nm}$ and $0.46 \mathrm{~nm}$, respectively. The latter value is even smaller than that of untreated $\mathrm{Si}_{3} \mathrm{~N}_{4}\left(R_{q}=1.41 \mathrm{~nm}\right)$. This means that the TMAH wet-etching process did not damage the $\mathrm{Si}_{3} \mathrm{~N}_{4}$ surface, but, on the contrary, reduced the surface roughness. The $\mathrm{SF}_{6}$ plasma dry-etching process almost fully destroyed the $\mathrm{Si}_{3} \mathrm{~N}_{4}$ ridge waveguide (with a height of $5 \mathrm{~nm}$ ). For these reasons the TMAH wet-etching process was selected to release the micro-cantilevers. It is noted that dry-etching with another etchant gas, i.e., Xenon difluoride $\left(\mathrm{XeF}_{2}\right)$, might yield better results than the one with $\mathrm{SF}_{6}$, since the etching selectivity between $\mathrm{Si}$ and $\mathrm{SiO}_{2}$ and $\mathrm{Si}_{3} \mathrm{~N}_{4}$ is much higher. Unfortunately, the $\mathrm{XeF}_{2}$ etchant was not available at MESA+ during the period in which the experiments were carried out. Table 4.3 summarized selectivity between $\mathrm{Si}$ and $\mathrm{SiO}_{2}$ and $\mathrm{Si}_{3} \mathrm{~N}_{4}$ with different etchants (two wet-etchants and two dry-etchants), wet-etchant TMAH and dry-etchant $\mathrm{XeF}_{2}$ show better selectivity [131].

Table 4.3 Comparison of selectivity between Si and $\mathrm{SiO}_{2}$ and $\mathrm{Si}_{3} \mathrm{~N}_{4}$ using different etchants to remove poly-Si sacrificial layer (data from Ref. [131]).

\begin{tabular}{lllll}
\hline Selectivity (etch rate) & $\mathrm{KOH}$ & $\mathrm{TMAH}$ & $\mathrm{SF}_{6}$ & $\mathrm{XeF}_{2}$ \\
\hline Etch type & Wet & Wet & Plasma & Vapor \\
Concentration & $40-50 \mathrm{wt} \%$ & $20-25 \mathrm{wt} \%$ & & \\
Temperature & $70^{\circ}-100^{\circ} \mathrm{C}$ & $90^{\circ} \mathrm{C}$ & $0^{\circ}-100^{\circ} \mathrm{C}$ & $20^{\circ} \mathrm{C}$ \\
$\mathrm{SiO}_{2}(\mathrm{~nm} / \mathrm{min})$ & 10 & 0.1 & 10 & 0 \\
$\mathrm{Si}(\mu \mathrm{m} / \mathrm{min})$ & $0.5-3$ & $0.5-1.5$ & $0.1-0.5$ & $0.1-10$ \\
$\mathrm{Si}_{3} \mathrm{~N}_{4}(\mathrm{~nm} / \mathrm{min})$ & 1 & 0.1 & 200 & 12 \\
\hline
\end{tabular}

The RIE process for transferring the grating pattern into the $\mathrm{Si}_{3} \mathrm{~N}_{4}$ layer was optimized for uniformity and aspect ratio. As a result, the $\mathrm{Si}_{3} \mathrm{~N}_{4}$ gratings were etched in an $\mathrm{O}_{2}(10 \mathrm{sccm}): \mathrm{CHF}_{3}(100 \mathrm{sccm})$ plasma at $40 \mathrm{mTorr}, 250 \mathrm{~W}$, for 2 min. Fig. 4.7 shows a high-resolution scanning electron microscopy (HR-SEM) image of a fabricated chip, exhibiting a grating (zoomed inset) with aspect ratio, period and uniformity as desired. However the suspended cantilever has a relatively large initial bending of around $3 \mu \mathrm{m}$, owing to tensile stress in the Pd layer. With such a large initial bending, the overlap of 
the cantilever with the evanescent tail of the GWG modes is too small for sensing purposes. For this reason we applied an extra step, $\mathrm{O}_{2}$ plasma cleaning, to oxidize the top of the Pd layer, which introduces a (compressive) surface stress leading to a strong decrease of the initial bending.
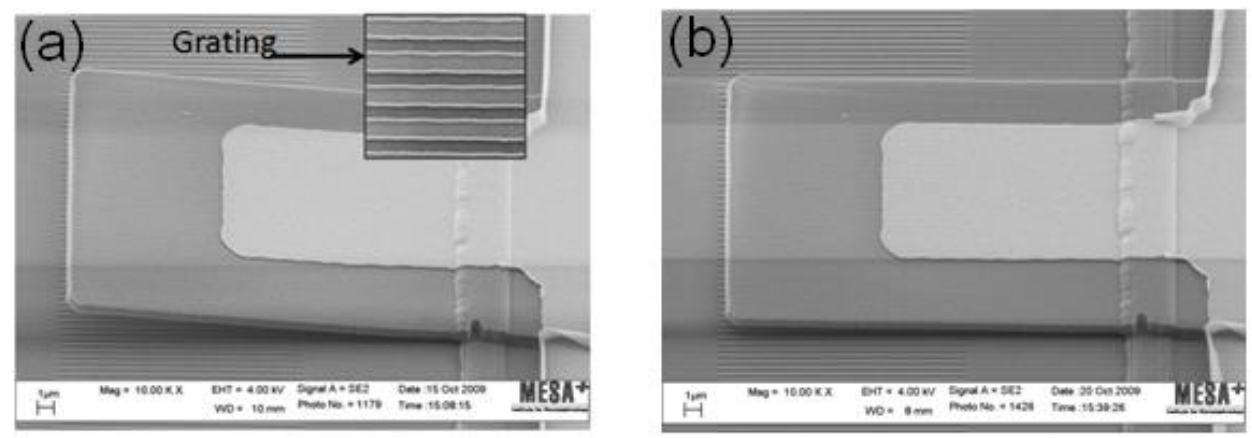

Fig. 4.7 High-resolution scanning electron microscopy (HR-SEM) image of a fabricated chip (a), grating (zoomed inset) with period of $490 \mathrm{~nm}$, fabricated by laser interference lithography (LIL), and suspended cantilever released by $T M A H$ wet etching solution. Reduction of initial bending was obtained by $\mathrm{O}_{2}$ plasma treatment (b).

For the optical characterization, sensor chips need to be separated from the wafer. If simple cleaving is used for this, the end facets are rough and sometimes ill-defined and accordingly, coupling light from a fiber into the chip is inefficient and in many cases virtually impossible. Therefore, a new technique has been developed for the fabrication of smooth end faces. The technique consists of the following steps (as shown in Fig. 4.8.):

* Reactive ion etching (RIE) to define the end facet for the waveguiding part of the structure

* Dicing of the chip at a safe distance from the above end-facet. (At this stage the cantilevers have not yet been released and hence are not susceptible to damage by the relatively rough dicing process.)

* TMAH etching of $\mathrm{Si}$, simultaneously releasing the cantilevers and removing part of the $\mathrm{Si}$ substrate to separate the chips, thus enabling fiber coupling. 


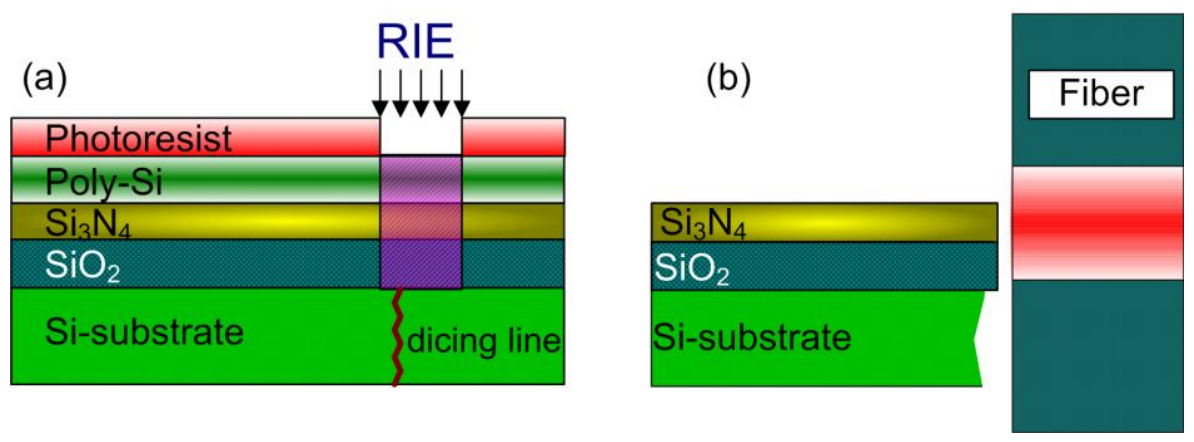

Fig. 4.8 Illustration of a new technique for improving the facet quality: (a) the reactive ion etching (RIE) and dicing steps, which are followed by TMAH etching of Si (b).

Figure 4.9 shows the optical microscopic images of facets achieved by the cleaving method (left) and by the new technique (right), demonstrating a significant improvement. The latter technique leads to a measured fiber-chip coupling efficiency of $9 \pm 0.3 \%$ at a wavelength of $1520 \mathrm{~nm}$, which indicates a relatively efficient fiber-chip coupling as it is close to the calculated value of $10.7 \%$.
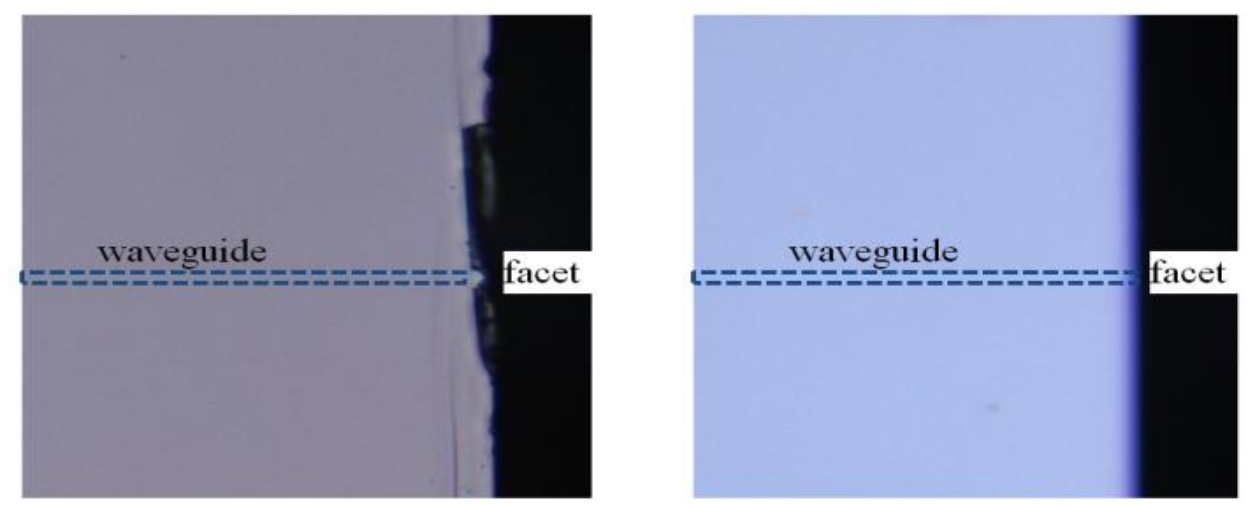

Fig. 4.9 Optical microscopic images of facets: (left) a rough and cracked cleaved facet and (right) a smooth facet obtained by the new technique. 


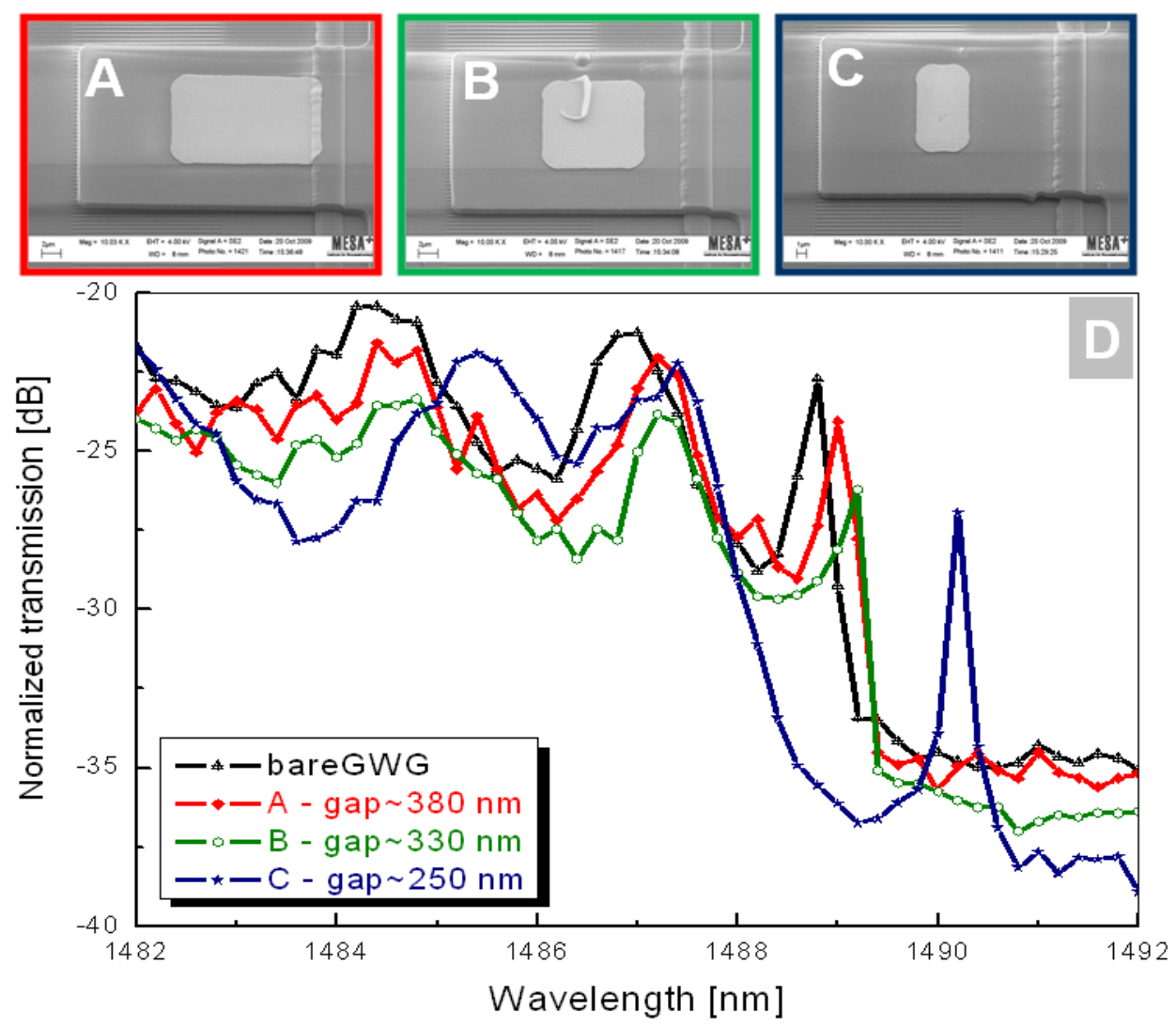

Fig. 4.10 Fabricated chips with $O_{2}$ plasma treatment $(A, B, C)$ and their measured transmission spectra (D) of a 250-period grating, with an $800 \mathrm{~nm}$ thick cantilever suspended above it. Gaps between the GWG and the cantilever in $A, B, C$ are different, owing to different metal pad lengths leading to differences in stress and, so, in initial bending.

For preliminary optical characterization, a series of cantilevers with varying metal-pad lengths (i.e., $15 \mu \mathrm{m}, 10 \mu \mathrm{m}$ and $5 \mu \mathrm{m}$ ) was fabricated so that samples were obtained having different surface stress, and hence different initial bending of the cantilevers. This resulted in different gaps between the GWG and the cantilever, before as well as after the $\mathrm{O}_{2}$ plasma treatment. The results of this process are shown in Fig. 4.10 (A, B, C). The gaps between cantilever and GWG, which decrease with the decreasing of metal area, are about $380 \mathrm{~nm}, 330 \mathrm{~nm}$ and $250 \mathrm{~nm}$, respectively. The difference in gaps leads to different spectral shifts of the transmission spectra as shown in Fig. 4.10 (D). The first resonance peak next to the band edge (at 
$\sim 1489 \mathrm{~nm}$ ) of the bare grating (i.e., no suspended cantilever present) is at a wavelength of $1488.8 \mathrm{~nm}$. This peak is shifted to wavelengths of $1489 \mathrm{~nm}$, $1489.2 \mathrm{~nm}$ and $1490.2 \mathrm{~nm}$ for gaps of $\sim 380 \mathrm{~nm}, \sim 330 \mathrm{~nm}$ and $\sim 250 \mathrm{~nm}$, respectively. These experimental results are in agreement with simulation results [109] and give good confidence in the read-out principle of the device.

Using $\mathrm{O}_{2}$ plasma for tuning the intial bending of Pd-coated cantilevers enabled sensing of beam deflection, but disabled hydrogen sensing. Solutions to achieve cantilevers with a pure Pd layer and a low initial bending (i.e., $100 \mathrm{~nm}$ ), which are able to absorb hydrogen and cause mechano-optical sensing effects, are presented in the next chapter (Chapter 5).

\subsection{Conclusions}

We have presented design considerations and fabrication-related aspects of a mechano-optical sensor for hydrogen gas, based on microcantilevers suspended above a grated waveguide. An optimized process that allows the fabrication of integrated GWG-cantilever sensors has been developed. Integrated devices with good-quality gratings and low initial bending cantilevers were realized, and a new technique for improving the quality of the end facet has been developed successfully. The presented experimental results prove the potential of the used read-out principle for cantilever deflection, with the integrated GWG-cantilever set-up, for hydrogen sensing. 


\section{Mechano-optical hydrogen sensor: proof of concept}

We demonstrate a proof of concept of a novel and compact integrated mechano-optical sensor for $\mathrm{H}_{2}$ detection based on a microcantilever suspended above a $\mathrm{Si}_{3} \mathrm{~N}_{4}$ grated waveguide. The fabricated devices are mechanically and optically modeled and characterized. Sensing operation of the sensor is demonstrated with $1 \% \mathrm{H}_{2}$ in $\mathrm{N}_{2}$. The error in detection of the cantilever bending induced by absorption of $\mathrm{H}_{2}$ is estimated to be approximately $10 \mathrm{~nm}$. Significantly improved sensitivity (down to $\sim 33 \mathrm{pm}$ ) is expected for reduced initial bending of the micro-cantilever. The simulation and experimental results are in good agreement and provide a good guideline for further optimization of the sensor. Recommendations for reducing the initial bending of micro-cantilever are also discussed. 4

${ }^{4}$ Part of this chapter has been published as: So V. Pham, Meindert Dijkstra, Henk A. G. M. van Wolferen, Markus Pollnau, Gijs J. M. Krijnen, and Hugo J. W. M. Hoekstra, "Integrated mechano-optical hydrogen gas sensor using cantilever bending read-out with a $\mathrm{Si}_{3} \mathrm{~N}_{4}$ grated waveguide," Opt. Lett. 36, 3003-3005 (2011) 


\subsection{Introduction}

A sensor is a device that can recognize the presence of a specific stimulus and translate it into a measurable signal [1]. Microcantilever-based sensors, which have effectively been exploited for biological, chemical, and gas sensing applications, can be operated in either dynamic mode by monitoring the resonant frequency of the cantilever $(\mathrm{CL})$, or static mode by measuring its stress-induced deflection, as the target binds to the functionalized surface of the $C L[14,132-137]$. The deflection of the $C L$ can be determined by means of bulky deflection of an optical beam [56] or electrical, i.e., capacitive, piezoresistive, or piezoelectric read-outs [43, 54, 55]. Alternatively, fully integrated optical read-out with a $\mathrm{CL}$ waveguide was firstly presented in 2006 by Zinoviev et al. [138] and in the following three years by other groups [139-142]. In 2008, we proposed a novel integrated optical read-out of singly-clamped $\mathrm{CL}(\mathrm{sCL}$ ) bending with a grated-waveguide (CWG) optical cavity and presented preliminary simulation results [109]. Recently, such integrated mechano-optical GWG-SCL devices have been fabricated and demonstrated as a proof of concept of the read-out [89].

In the study presented in this chapter, we aim at applying the proposed integrated mechano-optical read-out to hydrogen gas sensing. For this purpose, a 30-nm-thick palladium receptor layer was sputtered onto the entire surface of the $\mathrm{SiO}_{2} \mathrm{CL}$. At room temperature and atmospheric pressure, palladium can absorb up to 900 times its own volume of hydrogen [143]. Absorption of $\mathrm{H}_{2}$ by $\mathrm{Pd}$ causes the $\mathrm{CL}$, suspended above the GWG, to curl down $[43,54,56]$, which narrows the GWG-CL gap, $g$, and leads to a stronger interaction between the $\mathrm{CL}$ and the GWC evanescent modal field, which results in a red-shift of the transmission spectrum.

Functionalizing the $\mathrm{SiO}_{2} \mathrm{CL}$ by depositing the absorptive palladium film onto it causes an initial bending of the bilayer $\mathrm{CL}$ due to difference in their residual stresses [89]. In the fabrication batch presented in Chapter 4 (published in Ref. [89]), an oxygen-plasma treatment was strategically applied as a means to reduce such an initial bending of the bilayer $\mathrm{SCL}$. As a side effect, the palladium film was oxidized and no longer able to bind $\mathrm{H}_{2}$. Therefore, a solution to achieve a low initial bending of the $\mathrm{CL}$ without deactivating the functionality of the palladium film is required. One of our proposed solutions consisted of using devices with a doubly-clamped $\mathrm{CL}-\mathrm{a}$ so-called bridge- in addition to the sCLs as mentioned in Chapter 4. Initial 
bending of the cantilever and bridge are simulated and verified by experiments.

This chapter is organized as follows. Section 5.2 describes the experimental setup for $\mathrm{H}_{2}$ sensing. In Section 5.3 results related to mechanical characterization and sensing performance are presented and discussed. Structures for a sensor operating at high sensitivity are proposed and presented in Section 5.4. The chapter ends with conclusions (Section 5.5). It is noted that in this chapter the term cantilever $(C L)$ is used as a general term referring to both singly clamped cantilever $(\mathrm{SCL})$ and doubly clamped cantilever $(\mathrm{dCL})$.

\subsection{Experimental setup}

A three-dimensional (3D) schematic of the structure and the cross-section of the GWG-micro-bridge device are shown in Fig. 5.1a and Fig. 5.1b. All relevant device dimensions remain the same as in Ref. [12], except that here we choose an aimed gap of $g_{o}=200 \mathrm{~nm}$ and a bridge length of $L_{b}=65 \mu \mathrm{m}$. A $200 \mathrm{~nm}$ thick gap is chosen as compromise to attain a (reasonably) high sensitivity, on the one hand, and to have a not too small sensing range, on the other hand. A polydimethylsiloxane (PDMS) chamber was placed on top of the device and connected to gas bottles with pure $\mathrm{N}_{2}$ and a $1 \% \mathrm{H}_{2}-\mathrm{N}_{2}$ mixture through mass-flow controllers. The optical performance of the integrated device was monitored using a tunable laser source (Agilent 8164B) with a resolution of $1 \mathrm{pm}$ and an InGaAs photo-detector.

Table 5.1 Notations used in this chapter.

\begin{tabular}{lll}
\hline & Singly-clamped CL $(\mathrm{sCL})$ & Bridge \\
\hline Upper layer, Pd & $L_{1, s C L}, w_{1, s C L}, t_{1, s C L}, E_{1}, \alpha_{\tau_{1}}$ & $L_{1, b}, w_{1, b}, t_{1, b}, E_{1}, \alpha_{\tau_{1}}$ \\
Lower layer, $\mathrm{SiO}_{2}$ & $L_{2, s C L}, w_{2, s C L}, t_{2, s C L}, E_{2}, \alpha_{\tau_{2}}$ & $L_{2, b}, w_{2, b}, t_{2, b}, E_{2}, \alpha_{\tau_{2}}$ \\
\hline $\begin{array}{lll}\text { Initial gap (thickness of poly- } \\
\text { Si sacrificial layer) }\end{array}$ & $g_{0}$ & $g_{0}$ \\
Initial bending & & $\delta_{o, b}$ \\
Resulting gap & $\delta_{o, s C L}$ & $g_{\mathrm{b}}$ \\
\hline
\end{tabular}




\subsection{Results and discussion}

Response of the sensor with different gaps was simulated using the bidirectional eigenmode propagation method, as shown in Fig. 5.1c [109]. The results indicate three sensitivity regions of the sensor, depending on the overlap of the $\mathrm{CL}$ with the evanescent tail of the GWG modes.

Initial bending $\delta_{0 c}$ of the tip of the singly-clamped bilayer CLs was predicted using the Timoshenko formulas [112-114] described in (Eq. 5.1), considering the dimensions and material properties [126] of the CLs summarized in Table 5.2.

$$
\delta_{0 c}=L_{c}^{2} \frac{3 w_{1} w_{2} E_{1} E_{2} t_{1} t_{2}\left(t_{1}+t_{2}\right)\left[\left(\frac{\sigma_{01}}{E_{2}}-\frac{\sigma_{02}}{E_{1}}\right)+\left(\alpha_{\tau_{1}}-\alpha_{\tau_{2}}\right) \Delta \tau\right]}{\left(w_{1} E_{1} t_{1}^{2}\right)^{2}+\left(w_{2} E_{2} t_{2}^{2}\right)^{2}+2 w_{1} w_{2} E_{1} E_{2} t_{1} t_{2}\left(2 t_{1}^{2}+3 t_{1} t_{2}+2 t_{2}^{2}\right)}
$$

where $\delta_{0 c}, L_{c}, w_{i}$, and $t_{i}$ are the initial bending, length, width, and thickness of the CLs; $E_{i}, \sigma_{O}$, and $\alpha_{\tau_{i}}$ are Young's modulus, the residual stress and thermal expansion coefficient of material $i$, with the subscripts $i=1$ and 2 representing the $\mathrm{Pd}$ and $\mathrm{SiO}_{2}$ layers, respectively. The residual stresses of the films were determined based on Stoney's formula $\sigma_{0}=E_{s} t^{2} /\left[6 R\left(1-v_{s}\right)\right]$, where $E_{s}$ and $v_{s}$ are Young's modulus and the Poisson ratio of the substrate, and $R$ is the radius of curvature, obtained by measuring the wafer curvature, $1 / R$, due to deposition of the film on a silicon substrate [1]; their values are $\sigma_{o 1} \sim 1000 \mathrm{MPa}$ (tensile stress) and $\sigma_{02} \sim-310 \mathrm{MPa}$ (compressive stress) for $\mathrm{Pd}$ and $\mathrm{SiO}_{2}$ films, respectively.

Table 5.2 Dimensions and material properties of the CLs [126, 144].

\begin{tabular}{lll}
\hline Materials & $\mathrm{Pd}(1)$ & $\mathrm{SiO}_{2}(2)$ \\
\hline Length $L_{c}(\mu \mathrm{m})$ & 30 & 30 \\
Width $w(\mu \mathrm{m})$ & 20 & 20 \\
Thickness $t(\mu \mathrm{m})$ & 0.03 & 0.8 \\
Young modulus $E(\mathrm{GPa})$ & 121 & 61 \\
Residual stress $\sigma_{o}(\mathrm{MPa})$ & 1000 & -310 \\
Thermal expansion coefficient $\alpha\left(10^{-6} / \mathrm{K}\right)$ & 11.6 & 0.55 \\
\hline
\end{tabular}



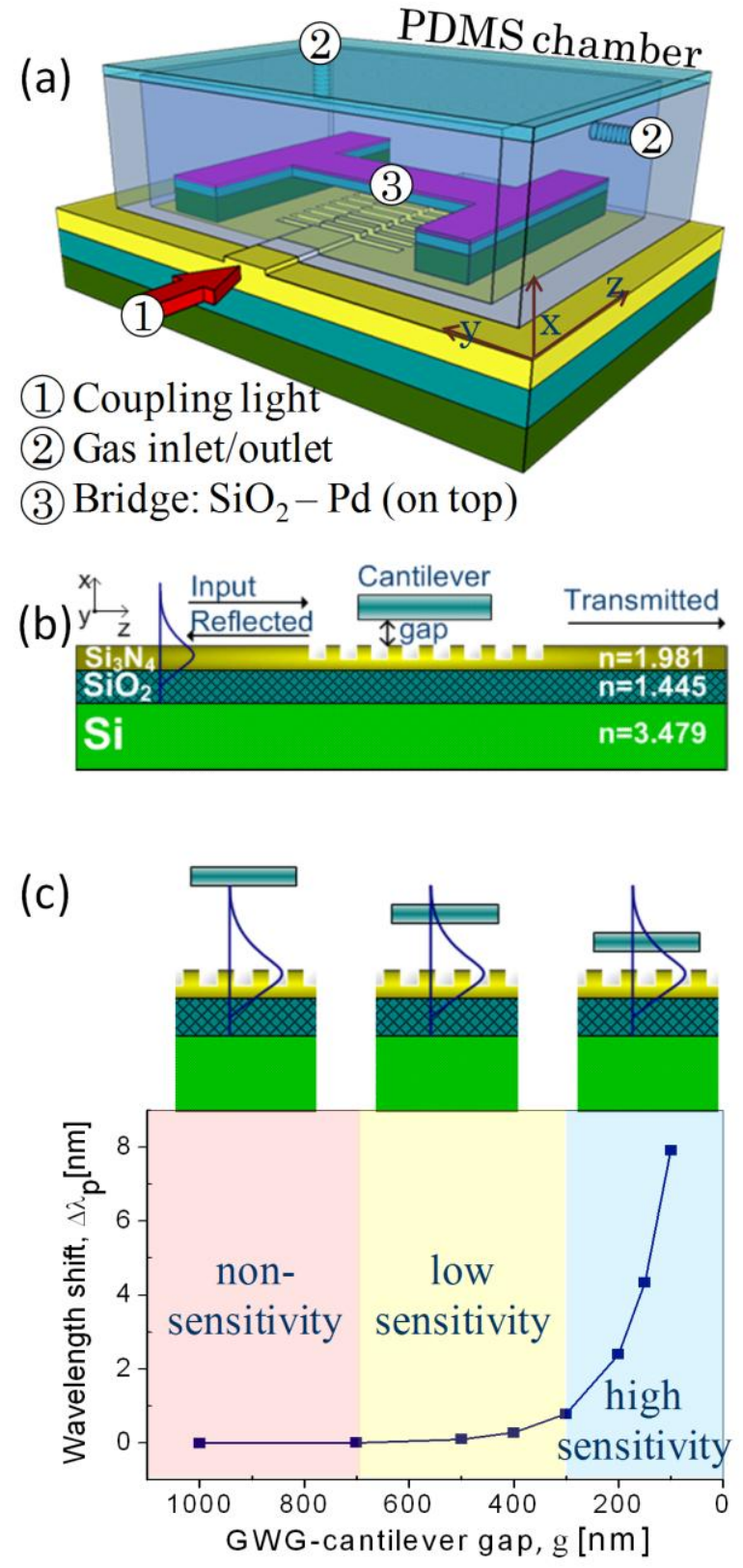

Fig. 5.1 (a) 3D schematic of the structure with a PDMS chamber serving as a reaction environment for $\mathrm{H}_{2}$ sensing, (b) cross-section of the CWC-CL device, and (c) simulated resonant wavelength shift for various gap sizes, illustrating three operating regions of the sensor. 
The residual stress acting on the bilayer $\mathrm{CLs}$ is equivalent to an accumulated force $F_{O}$ acting on the tip of the $s C L$ or at the middle of the bridge and, thus, the initial deflections of the $\operatorname{CLs}\left(\delta_{0 c}, \delta_{0 b}\right)$ are alternatively expressed as Eqs. (Eq. 5.2) and (Eq. 5.3) below [114, 145]. Therefore, once the initial bending of the $s \mathrm{CL}, \delta_{0 c}$, was calculated by (Eq. 5.1), the initial bending of the middle of bridge, $\delta_{0 b}$, was then deduced using (Eq. 5.4).

$$
\delta_{0 c}=\frac{F_{0} L_{c}^{3}}{3 E I},
$$

and

$$
\delta_{0 b}=\frac{F_{0} L_{b}^{3}}{192 E I},
$$

leading to

$$
\delta_{0 b} \sim\left(\frac{L_{b}}{4 L_{c}}\right)^{3} \delta_{0 c},
$$

with $I=w t^{3} / 12$, the moment of inertia of the CLs having a rectangular crosssection.

For comparison, finite-element simulations of the $\mathrm{CLs}$ were carried out using the INTELLISUITE software package [126], as shown in Fig. 5.2a (only the bridge structure is shown here). The initial bending of the fabricated $s \mathrm{CL}$ and bridge was characterized using a white-light interferometer. Fig. 5.2b shows a 3D image of the fabricated bridge, as recorded with a white-light interferometer, indicating an initial bending (upwards, i.e., away from the GWG structure) of approximately $500 \mathrm{~nm}$. The analytically calculated, numerically simulated, and experimentally measured values of the initial deflections are in good agreement and summarized in Table 5.3. The table also shows the resulting gap, $g=g_{0}+\delta_{0}$, of the GWG-sCL/bridge devices. The GWG-SCL device is not suitable for $\mathrm{H}_{2}$ sensing, because the gap $g \sim 3155$ $\mathrm{nm}$ is in the insensitive region of the sensor. On the contrary, the GWGbridge device is able to detect $\mathrm{H}_{2}$, even though the gap $g \sim 700 \mathrm{~nm}$ is in the low-sensitivity region of the sensor, as predicted in Fig. 5.1c. This initial bending leads to a lower sensitivity at low $\mathrm{H}_{2}$ concentrations. 
Table 5.3. Initial bending $\delta_{0}$ of the CLs and the final fabricated gap $g=g_{0}+\delta_{0}$, and sensitivity for thermal drift $\Delta \delta / \Delta \tau$.

\begin{tabular}{lll}
\hline & $s C L$ & Bridge \\
\hline Length $(\mu \mathrm{m})$ & 30 & 65 \\
Calculated $\delta_{0}(\mathrm{~nm})$ & 2977 & 473 \\
Simulated $\delta_{0}(\mathrm{~nm})$ & 3196 & $\sim 510$ \\
Measured $\delta_{0}(\mathrm{~nm})$ & $\sim 2955$ & $\sim 500$ \\
Final fabricated gap $g(\mathrm{~nm})$ & $\sim 3155$ & $\sim 700$ \\
Calculated $\Delta \delta / \Delta \tau(\mathrm{nm} / \mathrm{K})$ & $\sim 2.5$ & $\sim 0.4$ \\
\hline
\end{tabular}
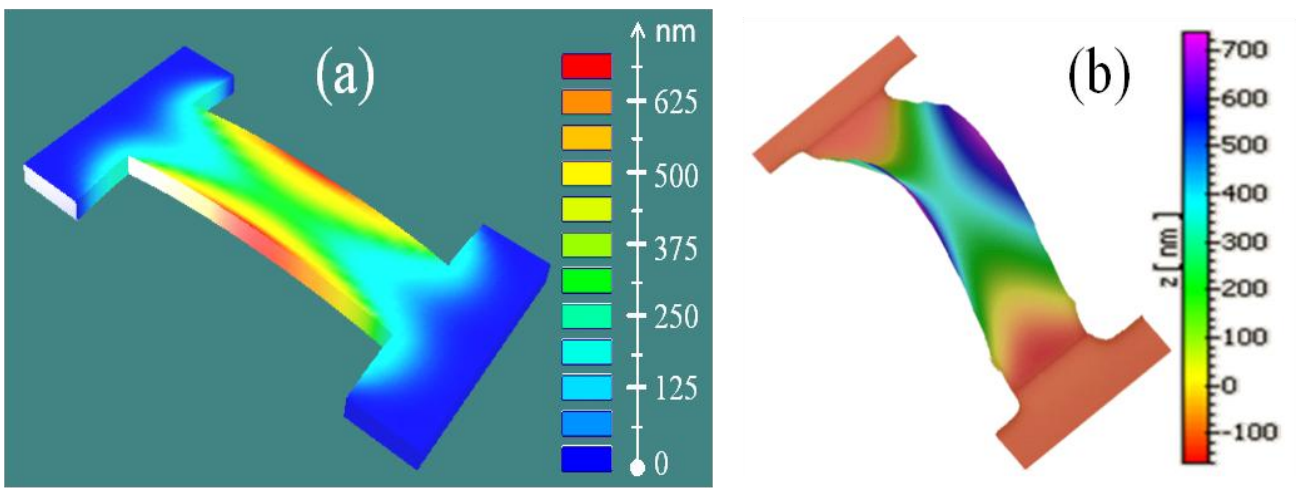

Fig. 5.2 Initial bending of the bridge due to differences in residual stress of the films: (a) numerical simulation using INTELLISUITE software package, (b) experimental result attained by a white-light interferometer, showing an initial up-bending of $\sim 500 \mathrm{~nm}$ at the center of the micro-bridge.

Prior to supplying $\mathrm{H}_{2}$ gas to the measurement chamber, $\mathrm{N}_{2}$ gas was flushed in during $15 \mathrm{~min}$ with a flow rate of $0.5 \mathrm{sccm}$ and optical transmission curves were captured repeatedly every minute. The results showed a stable and reproducible resonant peak at $\lambda_{p}=1496.631 \pm 0.001 \mathrm{~nm}$ (see Fig. 5.3a, curve at $t=0$ ), indicating that such a flow rate did not cause any side effects or mechano-optical vibrations. Noise was removed from the spectrum using low-pass filtering in the Fourier domain, enabling accurate and efficient determination of changes in $\Delta \lambda_{p}=\lambda_{p}(t)-\lambda_{p}\left(t_{o}\right)$.

Next we supplied the $\mathrm{H}_{2}(1 \%)-\mathrm{N}_{2}$ mixture at a flow rate of $0.5 \mathrm{sccm}$ for a longer period of time, during which the transmission spectrum was 
monitored (see Fig. 5.3a). The shift $\Delta \lambda_{p}$ depends almost linearly on time (Fig. $5.3 \mathrm{~b}$, left-hand side), which can be explained partly by noting that the effect of the initially rapid change of the gap size, $g$, is compensated by lower values of $\partial \lambda_{p} / \partial g$ at larger gap size. After 3.5 hours the flow of the $\mathrm{H}_{2}(1 \%)$ $\mathrm{N}_{2}$ mixture was switched off and replaced again by a pure $\mathrm{N}_{2}$ flow, leading to desorption. Fig. 5.3b (right-hand side) shows the resulting peak shifts during a four-hour period. It can be concluded that the desorption takes place at a much lower rate of $\sim 50 \%$ than the absorption process, and full desorption is not achieved during the monitoring period of time.

The result provides a proof of concept of a novel and compact integrated mechano-optical sensor, which underlines the feasibility of this type of sensor.
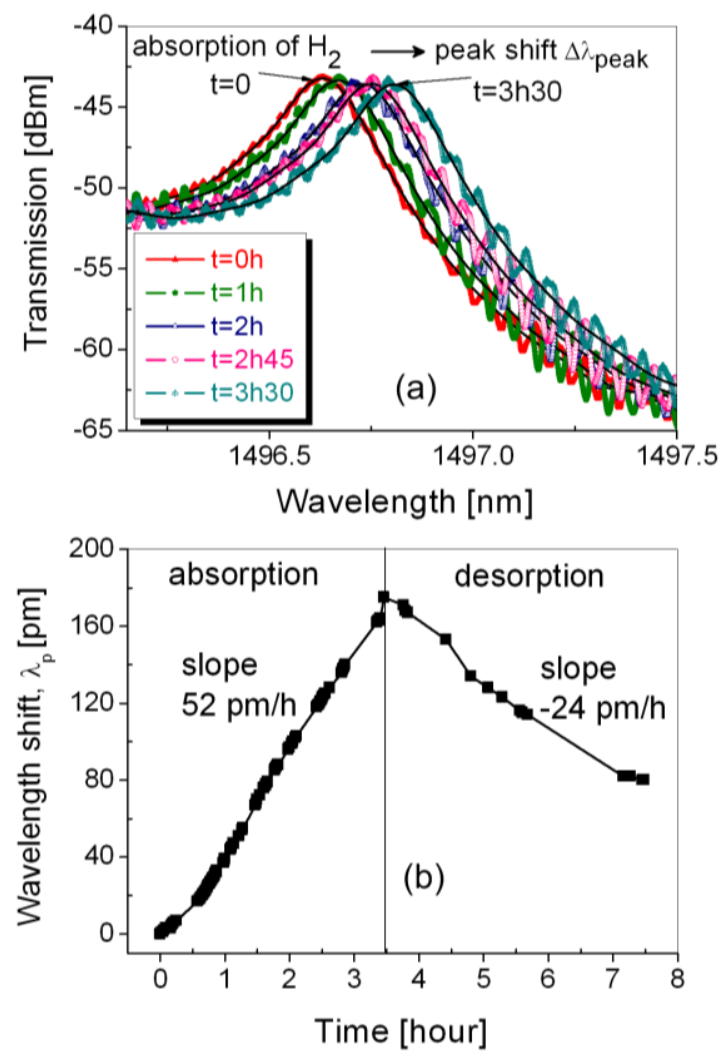

Fig. 5.3 (a) Transmission curves of the device in response to the absorption (filtered and unfiltered curves) and (b) the amount of wavelength shift $\Delta \lambda_{p}$ versus the reaction time: absorption (left-hand side) and desorption (righthand side). 
In practice, operation of the sensor is also affected by environmental changes due to the fluctuations in temperature, $\Delta \tau$. The difference in the thermal expansion coefficients between $\mathrm{Pd}$ and $\mathrm{SiO}_{2}$ causes a deflection change of $\sim 2.5 \mathrm{~nm} / \mathrm{K}$ for the $\mathrm{sCL}$ and $\sim 0.4 \mathrm{~nm} / \mathrm{K}$ for the bridge, which is theoretically estimated by omitting the quantity $\sigma_{01} / E_{1}-\sigma_{02} / E_{2}$ and retaining the quantity $\left(\alpha_{\tau_{1}}-\alpha_{\tau_{2}}\right) \Delta \tau$ in Eq. 5.1 with the parameters listed in Table 5.2. In addition, a temperature change also leads to a spectral shift of the grated-waveguide read-out. The resonant wavelength peak due to the temperature variation is measured, as shown in Fig. 5.4. The result shows a linear relationship $\Delta \lambda_{p}(\tau)$ with a slope $\Delta \lambda_{p} / \Delta \tau=16 \mathrm{pm} / \mathrm{K}$.

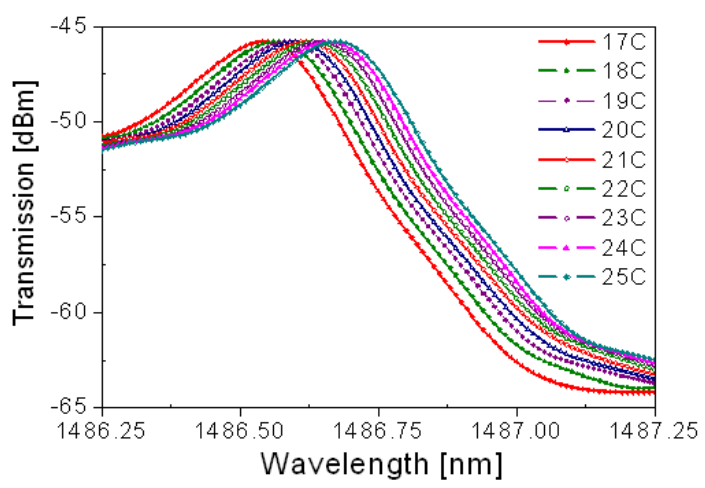

Fig. 5.4 Temperature dependence of the integrated optical read-out; the change of resonant wavelength shift is $16 \mathrm{pm} / \mathrm{K}$.

\subsection{Structures for a sensor operating at high sensitivity}

So far, the main purpose of fabricating a functioning integrated mechanooptical sensor has been fulfilled. For this purpose, preliminary design parameters were chosen with the considerations presented in Chapter 4. In fact, the initial bending of the bimaterial cantilevers ( $\mathrm{s} C \mathrm{~L}$ and bridge) limited the operation of the sensor, in particular, its sensitivity. Therefore, solutions for the improvement of initial bending are required.

The partly covered cantilevers mentioned in Chapter 4 have not been considered in this chapter since the initial bending is large and the sensor works in the low-sensitivity region where the absorption loss hardly plays any role. However, an optimized design for the sensor operating at high sensitivity should avoid absorption loss by the metal. Therefore the following proposed structures with a partly covered Pd layer are presented. The main 
idea for these structures is to use a proper combination of thicknesses, materials and (relative) length of the Pd layer for compensation of residual stress in the thin films.

A tri-layer stack of Pd/ PECVD-SiO2/ TEOS-SiO $\mathrm{SH}_{2}$ (tensile stress/ compressive/ less compressive) is proposed based on a combination of the situations depicted in Fig. 4.2 a and c (see Chapter 4). In details, firstly the $\mathrm{PECVD} \mathrm{SiO}_{2}$ (compressive stress)/ $\mathrm{TEOS} \mathrm{SiO}_{2}$ (less compressive stress) stack induces a downward bending (Fig. 4c) and then the coated Pd layer with tensile stress will tend to pull the PECVD $\mathrm{SiO}_{2} / \mathrm{TEOS} \mathrm{SiO}_{2}$ stack upward $\left(\mathrm{SiO}_{2}\right.$ compressive/ Pd-tensile, Fig 4.2a), and reduce the total down-ward bending of the tri-layer $\mathrm{CL}$. To confirm such a layer-combination concept, numerical simulations using the INTELLISUITE software package are performed for two partly covered $s C L s$ (not $d C L s$ ), of which their structural parameters are given in Table 5.4 and simulation results are shown in Fig. 5.5. The two considered tri-layer sCLs differ only in the thicknesses of the PECVD and TEOS $\mathrm{SiO}_{2}$ layers (their total thickness remains constant: $t_{P E C V D}+t_{T E O S}=0.8 \mu \mathrm{m}$ ). Depending on the thicknesses of the PECVD and TEOS $\mathrm{SiO}_{2}$ layers, the downward initial bending of the tri-layer $\mathrm{CLs}$ can be controlled to be from tens (Fig. 5.5a) to thousands of nanometers (Fig. 5.5b). 
Table 5.4 Structural parameters for simulation of the two proposed tri-layer sCLs (a) and (b). Blue boxed parameters remain the same, while yellow boxed ones are different for the two SCLs.

\begin{tabular}{|l|l|l|l|}
\hline Materials & $\begin{array}{l}\mathrm{Pd}(1) \\
\text { (top) }\end{array}$ & $\begin{array}{l}\mathrm{PECVD} \mathrm{SiO} \\
\text { (middle) }\end{array}$ & $\begin{array}{l}\mathrm{TEOS} \mathrm{SiO}_{2}(3) \\
\text { (bottom) }\end{array}$ \\
\hline Length $L_{c}(\mu \mathrm{m})$ & 15 & 30 & 30 \\
\hline Width $w(\mu \mathrm{m})$ & 10 & 20 & 20 \\
\hline \multirow{2}{*}{ Thickness $t(\mu \mathrm{m})$} & \multirow{2}{*}{0.03} & $0.05(\mathrm{a})$ & $0.75(\mathrm{a})$ \\
\cline { 2 - 4 } & & $0.3(\mathrm{~b})$ & $0.5(\mathrm{~b})$ \\
\hline
\end{tabular}
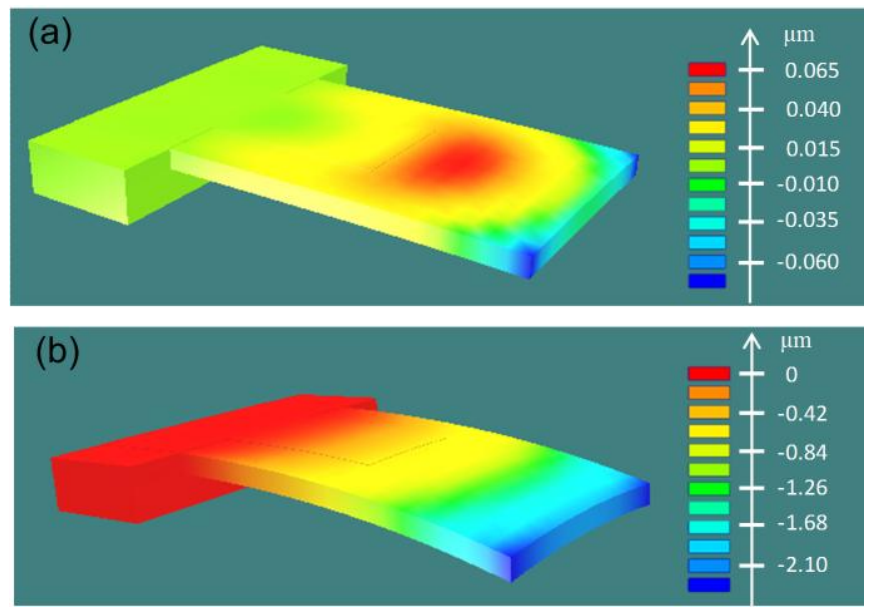

Fig. 5.5 Simulated down-ward initial bending of the two proposed tri-layer sCLs with structural parameters given in Table 5.4.

Given the desired CL-GWG initial gap of $200 \mathrm{~nm}$ for the sensor (i.e., $g_{o}=t_{S i}+\delta_{o} \approx 200 \mathrm{~nm}$, with $t_{S i}$ the thickness of the poly-Si sacrificial layer and $\delta_{o}(\leq 0)$ the (down-ward) initial bending of the tri-layer $\mathrm{CL}$ ) and the other chosen parameters (i.e., blue boxed parameters in Table 5.4), a (continuous) range of options for the parameters of the tri-layer $\mathrm{CL}$ can be chosen, two of these are mentioned below:

* Choose $t_{S i}=200 \mathrm{~nm}$ and control the thicknesses of the PECVD $\mathrm{SiO}_{2}$ and TEOS $\mathrm{SiO}_{2}$ layers such that there is zero downward bending of the tri-layer $\mathrm{CL}$, i.e., $\delta_{o} \approx 0$ (Fig. 5.6a). 
* If the thicknesses of the two oxide layers are chosen such that downward bending occurs one may calculate the magnitude of the latter and choose the thickness of the sacrificial layer according to $t_{S i}=g_{o}-\delta_{o} \approx 200 \mathrm{~nm}-\delta_{o}$ (Fig. 5.6b).

For actual realization of a correct gap, fine tuning, using experimental results, may be required.

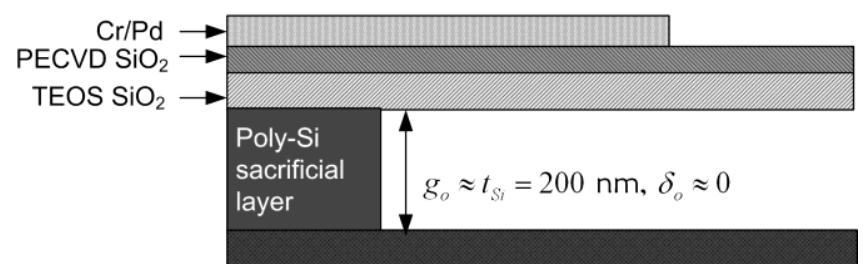

Substrate

(a)

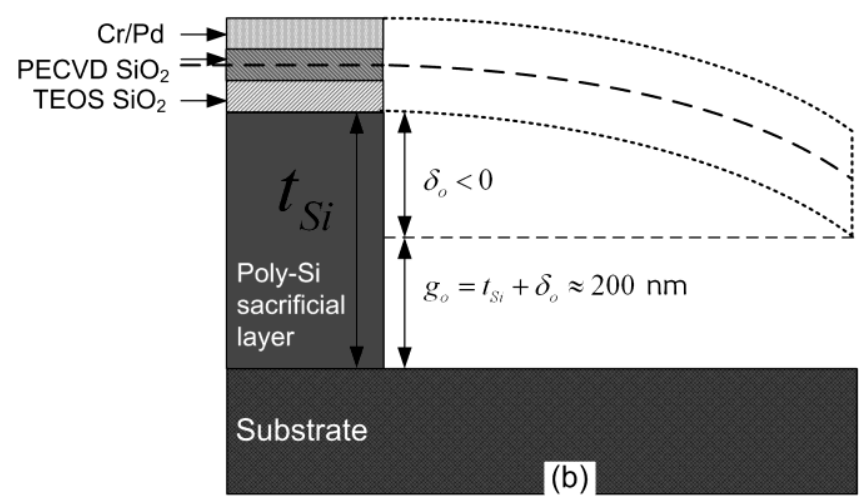

Fig. 5.6 Schematic 2D cross-section of a tri-layer CL, initial gap $g_{o} \approx 200 \mathrm{~nm}$ can be obtained by controlling the thicknesses of the PECVD and TEOS $\mathrm{SiO}_{2}$ layers in such a way that (a) $\delta_{o} \approx 0\left(t_{S i}=200 \mathrm{~nm}\right)$ or (b) $\delta_{o}=$ const $(<0), t_{S i}=g_{o}-\delta_{o} \approx 200 \mathrm{~nm}-\delta_{o}$. 


\subsection{Conclusions}

To conclude, we have demonstrated $\mathrm{H}_{2}$ sensing with a novel and compact integrated optical read-out scheme as a proof of concept for our mechanooptical sensor. It was found that the error in the peak shift, owing to noise and thermal fluctuations in the temperature-stabilized set-up, is around $1 \mathrm{pm}$. Then, using that $\Delta \lambda_{p} / \Delta g=0.1,0.8$, and $30 \mathrm{pm} / \mathrm{nm}$, at a gap of $g=700$, 500 , and $200 \mathrm{~nm}$, respectively (according to the theoretical curve in Fig. 5.1c), the error in detection of the gap is given by $10 \mathrm{~nm}, 1.25 \mathrm{~nm}$, and 33 pm for the aforementioned gap sizes, respectively. This new sensor type possesses a great potential as an element of a sensitive, on-chip multi-sensing system, provided that the gap between the GWG and the micro-cantilever, i.e., $\mathrm{s} C \mathrm{~L}$ and bridge, can be well controlled during fabrication. The sensing principle presented here could also be used for other specific surface reactions in a gaseous environment. For use in a liquid environment, e.g., label-free protein sensing exploiting an antigen-antibody reaction on the $C L$ surface, the stiction problem between $\mathrm{CL}$ and $\mathrm{CWG}$ caused by adhesive forces must be solved.

Further on, structures for obtaining low initial bending are proposed. For the bilayer $\mathrm{SiO}_{2} / \mathrm{Pd} \mathrm{CL}$, the initial bending is too large to be within reach of the evanescent field of the mode (total gap preferentially $200 \mathrm{~nm}$, smaller than $700 \mathrm{~nm}$ ). It is made plausible that with a tri-layer CL, an effective gap of $\sim 200 \mathrm{~nm}$ can be obtained by controlling the thicknesses of the PECVD and TEOS $\mathrm{SiO}_{2}$ layers to achieve a virtually zero initial bending or by increasing the thickness of the poly-silicon sacrificial layer adapted to the (down-ward) initial bending corresponding to a given thickness ratio. 



\section{Mechano-optical hydrogen sensor: analysis and optimization}

In chapters 4 and 5, we have proposed and demonstrated a novel and compact read-out principle for cantilever deflection using a grated waveguide optical cavity. The device, consisting of a micro-cantilever suspended above a $\mathrm{Si}_{3} \mathrm{~N}_{4}$ grated waveguide (GWG), is a potentially highly sensitive mechano-optical sensor for gas sensing. In this report we discuss the device principle and present results of a numerical investigation to optimize the read-out of such an integrated GWG-cantilever device using the bidirectional eigenmode propagation method and a grating mode solver. The results show that sensitivity of the device strongly depends on the length of the grated section, the cantilever width and to a lesser extent on the grating depth. ${ }^{5}$

${ }^{5}$ Part of this chapter is being prepared for publication as: S. V. Pham, R. M. de Ridder, and H. J. W. M. Hoekstra, "Numerical investigation and optimization of a mechano-optical sensor based on an optical grated-waveguide cavity," in preparation. 


\subsection{Introduction}

An introduction to the field has been given in Sections 4.1 and 5.1. In this chapter, we will first discuss the device operation principle, which will serve as a basis for considerations related to device optimization. As to the latter, we will try to find trends for the sensitivity of such an integrated OWG cantilever device as a function of device parameters in order to provide approximate, general guidelines for obtaining optimal device performance.

The rest of this chapter is organized as follows. Section 6.2 describes the device structure and parameters. Section 6.3 discusses the device sensing principle, sensitivity and the most relevant parameters to be varied for optimization of the read-out of cantilever deflection. In section 6.4 results are described and discussed. The paper ends with conclusions (section 6.5).

\subsection{Device structure and parameters}

The 3D schematic structure and the 2D cross-sections of the GWG-cantilever device are shown in Figs. 6.1a-c. Main parts of the device are the $\mathrm{Si}_{3} \mathrm{~N}_{4}$ grated waveguide and the $\mathrm{SiO}_{2}$ cantilever coated with a receptor layer for gas absorption. All dimensions and refractive indices are depicted in the figures and Table 6.1. In Table 6.1, the choice of the parameters, which are assumed to be fixed and those which are to be varied during the aforementioned optimization process, are indicated.

Table 6.1 Structural parameters of GWG-cantilever integrated system.

\begin{tabular}{llll}
\hline Component & Parameter & Value & Status \\
\hline \multirow{3}{*}{ Waveguide } & Ridge height, $h$ & $5 \mathrm{~nm}$ & fixed \\
& Ridge width, $w_{\text {wg }}$ & $5 \mu \mathrm{m}$ & fixed \\
& Thickness, $t_{\mathrm{wg}}$ & $275 \mathrm{~nm}$ & fixed \\
\hline \multirow{2}{*}{ Grating } & Period, $\Lambda$ & $490 \mathrm{~nm}$ & fixed \\
& Length, $L$ & $125 \Lambda, 250 \Lambda, 375 \Lambda$ & variable \\
& Groove depth, $d$ & $55,75 \mathrm{~nm}$ & variable \\
\hline CWG- & Gap, $g$ & $0-400 \mathrm{~nm}$ & variable \\
cantilever gap & & & \\
\hline cantilever & Width, $W$ & $20 \mu \mathrm{m}$, or $W / L=0.1-1$ & variable \\
& Thickness, $t$ & $800 \mathrm{~nm}$ & variable \\
\hline
\end{tabular}



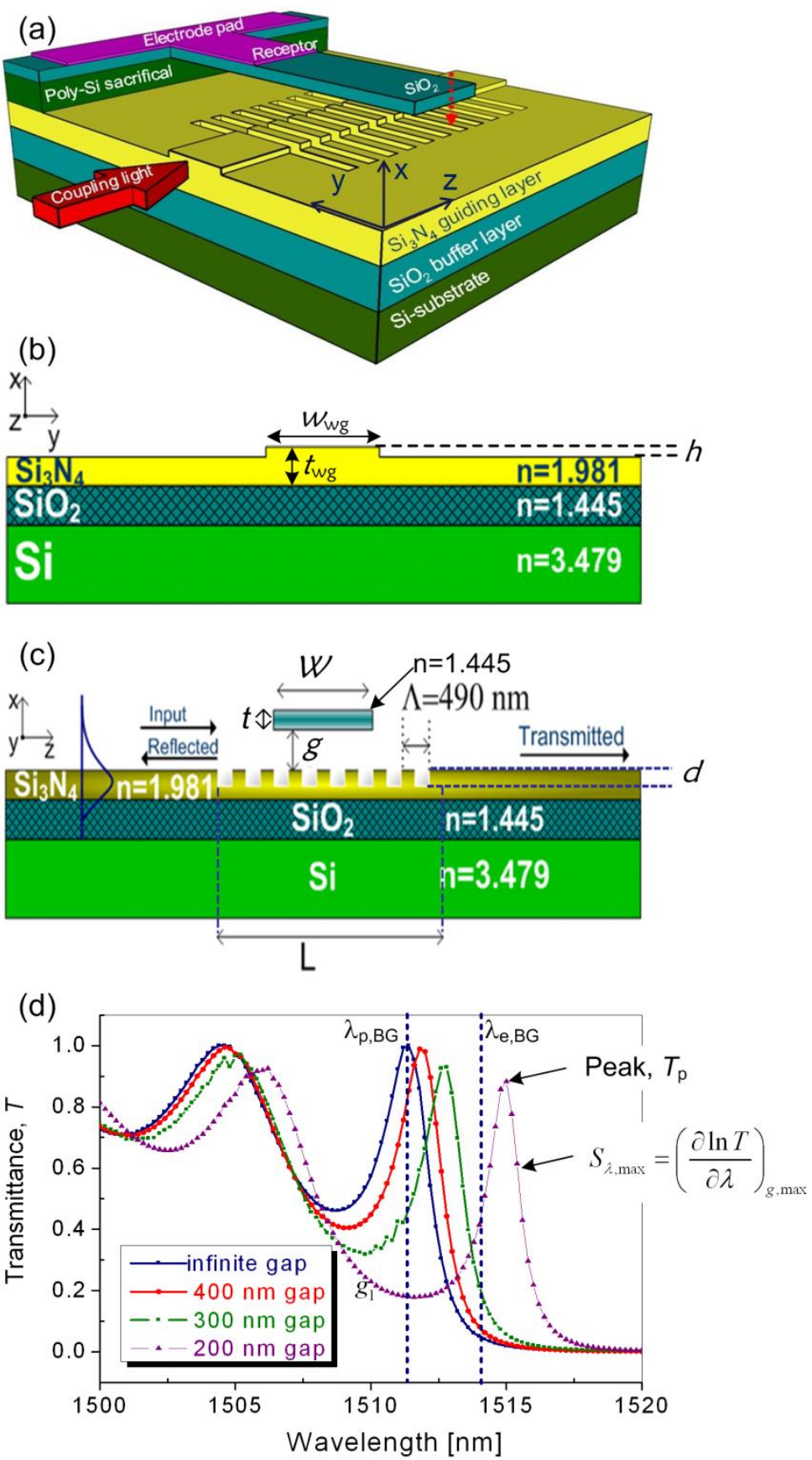

Fig. 6.1 The 3D schematic structure (a) and two cross-sections (b,c) of the GWG-cantilever device, and the simulated spectral shift due to varying the GWG-cantilever gap (d). 
It is assumed that of the above fixed device parameters the ridge width, $W_{w g}$, the grating period $(\Lambda)$ and the waveguide thickness, $t_{w g}$, are not very critical for device performance. The grating period has been chosen such that the grating stop band and its accompanying fringes are within reach of the used laser source. The relatively small ridge height $(5 \mathrm{~nm})$ may lead to losses at transitions between waveguiding sections, via radiation into lateral directions (along $y$, see Fig. 6.1b), which are not taken into account in the calculations presented here on the structure with reduced dimensionality. At the moment the magnitude of such losses nor the effect of the ridge height on these are not known to us; for the latter 3D calculations or detailed experiments would be required, both being beyond the scope of this chapter.

\subsection{Device analysis and sensitivity}

In this section the device principle is analyzed, expressions for the sensitivity are introduced and expressions which are helpful for the device optimization are derived. All computational results presented in this chapter are obtained using a grating mode solver (GMS) [76] for modal properties, and the bidirectional eigenmode propagation method (BEP) [95] for light propagation in the device. Both methods use dimensionality reduction from $3 D$ to $2 D$. For the $2 \mathrm{D}$ calculations, the geometry of the guiding part (the channel) is used. As the channel is defined by a guiding segment only $5 \mathrm{~nm}$ thicker than the adjacent (slab) sections it may be assumed that the dispersion curves $(\beta(\lambda))$ of grated sections are reproduced reasonably well. However, scattering losses, due to mode mismatch, in the two lateral directions (i.e., along $\pm y$, see Fig. 6.1) are fully neglected, which may introduce an (yet unknown) underestimation of the losses at such transitions.

With mode mismatch between two modes we mean any difference in modal field solutions (field or propagation constant). Mode mismatch always leads to modal reflection and, generally, also to scattering losses (to radiation modes). The extent of the latter depends strongly on the geometry of the considered waveguides and may vanish in, for example, a 1D setting (layered structure with plane wave fields).

Absorption of gas by the receptor layer (e.g., $\mathrm{H}_{2}$ by a Pd-layer) will cause the cantilever to curl down $[43,56]$, which narrows the GWG-cantilever gap, $g$, and leads to stronger interaction between the cantilever and the GWG evanescent field. This results into a shift of the transmission spectrum. 
This effect can be used for the detection of cantilever displacements (see Fig. 6.1d). For sensing we use the (sharpest) first peak left of the bandgap, i.e., the peak at the right hand side of Fig. 6.1d.

While varying the gap $g$ between cantilever and GWG, two regimes can be identified; regime 1 corresponds to a situation in which $\lambda_{p, C L}<\lambda_{e, B G}$, and regime 2 corresponds to $\lambda_{p, C L}>\lambda_{e, B G}$ (with $\lambda_{p, C L}$, the first resonant wavelength - near the band edge - of the cantilever-covered GWG, and $\lambda_{e, B G}$, the band edge wavelength of the bare grating), as will be discussed in detail in the following. For infinite $g$, corresponding to the bare GWC the resonance condition for the considered peak is given by [76]:

$$
\beta_{p, B G} L=\frac{L \pi}{\Lambda}(1-1 / M)=\pi(M-1),
$$

with $\beta_{p, B G}$ the modal propagation constant or wavenumber of the Bloch mode in the grating, at the first resonance adjacent to the band edge of the bare grating and $M(\equiv L / \Lambda)$ the number of grooves of the grating. Equation 6.1 is illustrated by Fig. 6.2 showing the band structure (calculated with the grating mode solver) of infinitely long gratings, both without and with an infinitely wide cantilever in close proximity $(g=200 \mathrm{~nm})$, where the first resonant state of a finite length $(L=125 \Lambda)$ grating without cantilever is shown as a dot. 

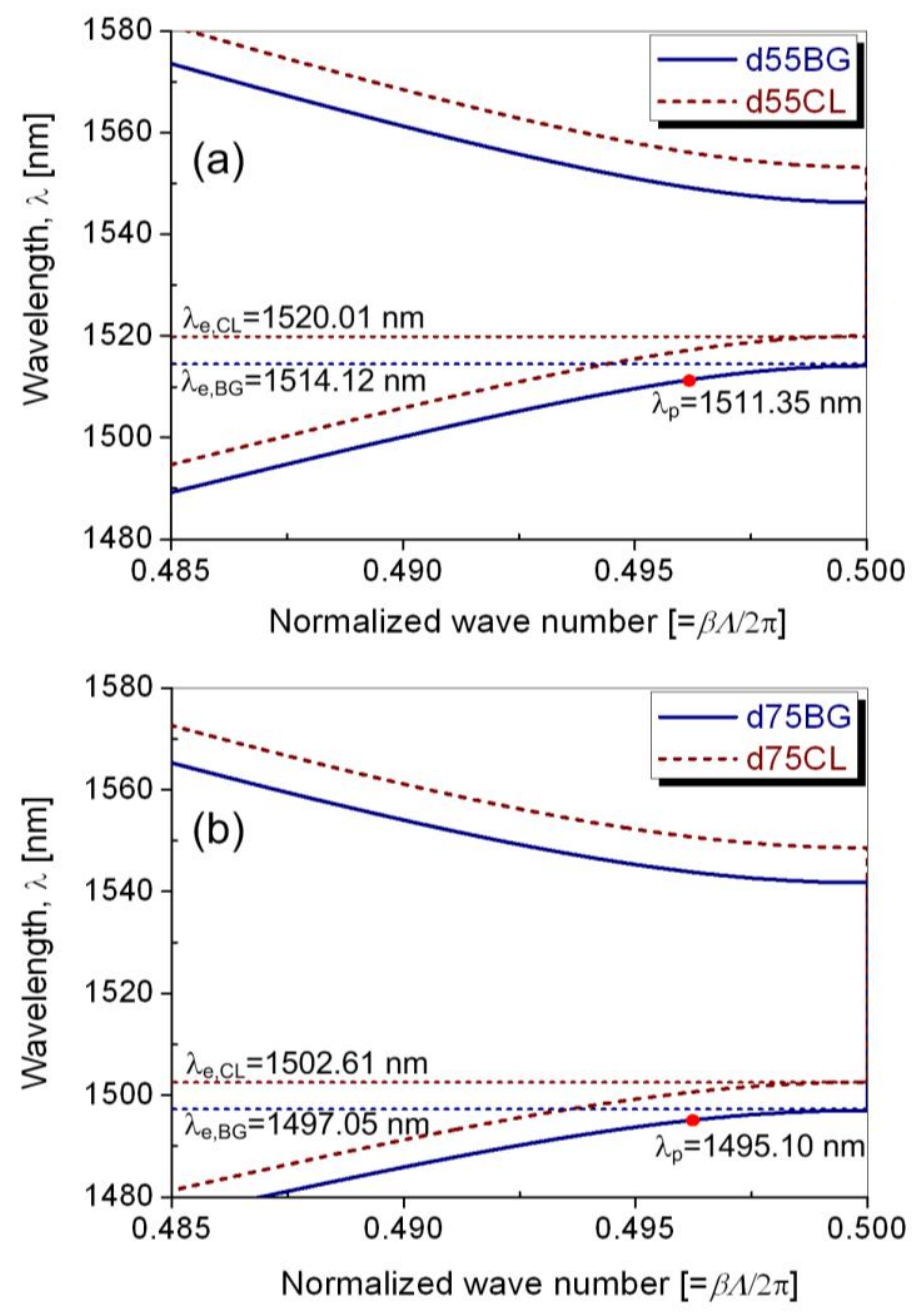

Fig. 6.2 Dispersion curves of an infinitely long GWC (solid blue line) and the same GWC completely covered by a cantilever in close proximity ( $g=200 \mathrm{~nm}$ ) (dashed brown line) for two values of the grating depth $d$ : (a) $d=55 \mathrm{~nm}$, wavelength at the band edge of the grating is $\lambda_{\text {edge }}=1514.12 \mathrm{~nm}$, and (b) $d=75 \mathrm{~nm}, \lambda_{\text {edge }}=1497.05 \mathrm{~nm}$. For both cases (a) and (b), the resonant state of a finite-length GWG of $L=125$ periods is indicated by a red dot, with $\lambda_{p}=1511.35 \mathrm{~nm}$ and $1495.10 \mathrm{~nm}$ for $d=55 \mathrm{~nm}$ and $75 \mathrm{~nm}$, respectively. 
Equation (6.1) relies among other things on the fact that phase shifts on reflection and transmission at the grating-WG transition can be assumed to be small [76]. The resonant wavelength of a finite-length bare grating, $\lambda_{p, B G}$ is indicated in Fig. 6.3a, by the lowest horizontal dashed line. For finite $g$ the modal dispersion curve for the GWG in the grated section shifts upwards, as shown later on (see Fig. 6.3a). If $g$ is not too small we arrive at a situation indicated by the middle horizontal dashed line in Fig. 6.3, with a resonance wavelength still outside the band gap of the bare grating. The approximate resonance condition is then given by:

$$
\beta_{B G}(L-W)+\beta W \cong \pi(M-1),
$$

where $\beta$ is the propagation constant of the Bloch mode of the GWG in the cantilever section. In the above we have neglected modal reflections and also phase shifts at structural transitions near the $\mathrm{CL}$. Note that in the above (regime 1) situation the resonance condition corresponds to two varying (as a function of $g$ ) propagation constants, contrary to the situation in regime 2 (see below) where only $\beta$ is varying, while $\beta_{B G}$ is fixed.

Equation (6.2) illustrates the behavior of the structure in regime 1. Since the condition of a small phase shift at structural transitions is not warranted, the approximation may be a bit crude. Equation 6.2 is not used further on in this chapter.

A different approximate resonance condition can be derived for the operation in regime 2, where owing to a further decreasing gap the resonance wavelength lies inside the band gap of the $B G$, and the real part of $\beta_{B G}$ becomes a constant $\left(\operatorname{Re}\left(\beta_{B G}\right)=\pi / \Lambda\right)$ ([92], chapter 4):

$$
2 \beta W+2 \varphi \cong 2 m \pi
$$

where $m$ is an integer, and where we took each of the two BC sections and corresponding structural transitions as a mirror with phase shifts upon reflection of $\varphi$. Neglecting any wavelength dependence of these phase shifts the wave number $\beta$ at resonance is a constant. In most interesting cases (low $g$, higher sensitivity) the device is operated in regime 2 , which is assumed in the expressions discussed below, unless stated differently. In 
regime 2 the modes in the $\mathrm{BC}$ are evanescent, so that the light tunnels through the $B G$ parts. The tunneling contributes (next to mode mismatch) to the mirror function of the BC parts for the Fabry-Perot cavity (which may be considered to have a length equal to the $\mathrm{CL}$ width). The nature of the modes (evanescent or propagating) for the two regimes and the different regions is indicated in Fig. 6.3b.
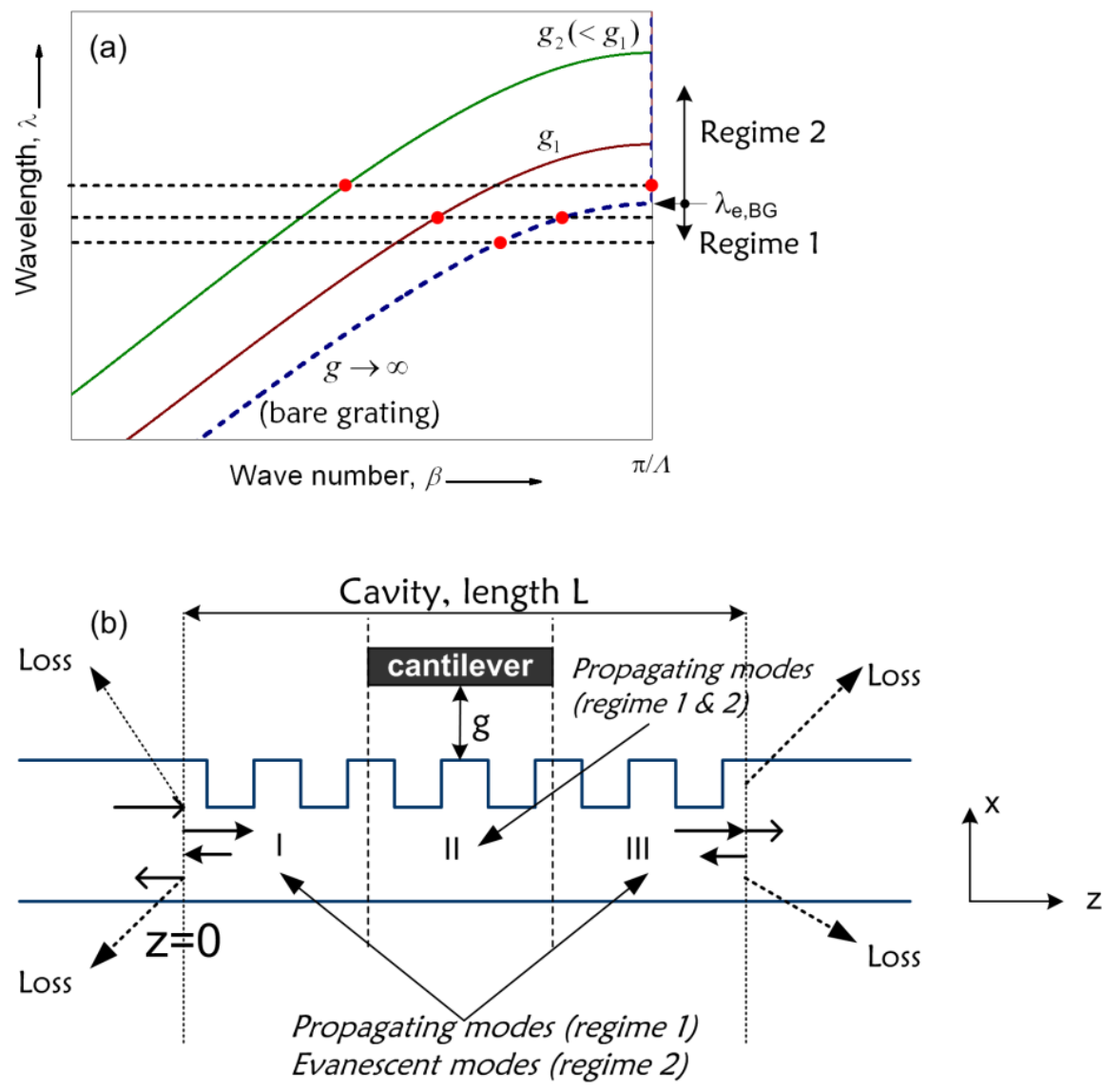

Fig. 6.3 (a) Schematic drawing of the relevant part of the band structure of a bare GWG and a GWG-cantilever system illustrating the two regimes discussed in the text. The red dots indicate the propagation constant(s) at resonant wavelengths for different values of g. (b) Schematic picture showing among others the nature of the modes for the two regimes. 
In order to be able to relate gap changes to resonance-wavelength shifts we consider the expression

$$
\left(\frac{\partial \lambda}{\partial g}\right)_{\beta} \cong A\left(e^{-2 \gamma g}-e^{-2 \gamma(g+t)}\right)=B e^{-2 \gamma g},
$$

which can be derived from ([92]; Eqs. 28 and 29 of chapter 2), considering the overlap of the evanescent field of the resonant mode with the cantilever. Here we assumed that the modal field is not altered very much by the cantilever, and we used that the cantilever movement causes index changes at distances $g$ and $g+t$ from the GWG. The parameters $A$ and $B$ (depending on the wavelength, structure, and corresponding modal field) can be shown to be negative, indicating indeed a shift upwards (larger wavelength) of the dispersion curves for decreasing values of $g$. In the above $\gamma$ is the approximate decay constant of the two dominant Floquet-Bloch components of the modal field, having $z$-dependencies given by $\exp (-i \beta z)$, and $\exp [-i(\beta-K) z] \approx \exp (i \beta z)$, where $K=2 \pi / \Lambda(\sim 2 \beta$, for modes near the band edge) is the wavenumber of the grating. Its value is given by $\gamma \approx \sqrt{\beta^{2}-k_{0}^{2}}$, with $k_{0}$ the free-space wavenumber of the light and we assumed an index for air equal to unity. Equation 6.4 can be used to deduce (approximately, as changes in the phase of modal reflections as a function of $g$ are ignored) wavelength shifts of peaks and the band edge in all cases that, as indicated by the subscript, $\beta$ is a constant (as in regime 2), discussed above. Equation 6.4 is illustrated by Fig. 6.4a showing the curve of $\left|\partial \lambda_{p} / \partial g\right|\left(=\left|(\partial \lambda / \partial g)_{\beta}\right|\right)$ versus $g$, from which the values $B(\approx-0.38)$ and $\gamma\left(\approx 4.8 \times 10^{-3} \mathrm{~nm}^{-1}\right)$ are derived and by Fig. $6.4 \mathrm{~b}$ showing the calculated wavelength corresponding to the lower edge of the band gap of a CWC with a cantilever above it, as a function of $g$, for $t=800 \mathrm{~nm}$. The linear dependence of $\ln \left[(-2 \gamma / B) \times\left(\lambda_{e, C L}-\lambda_{e, B G}\right)\right]$ follows from Eq. 6.4 by integration between $g$ and $g=\infty$. 

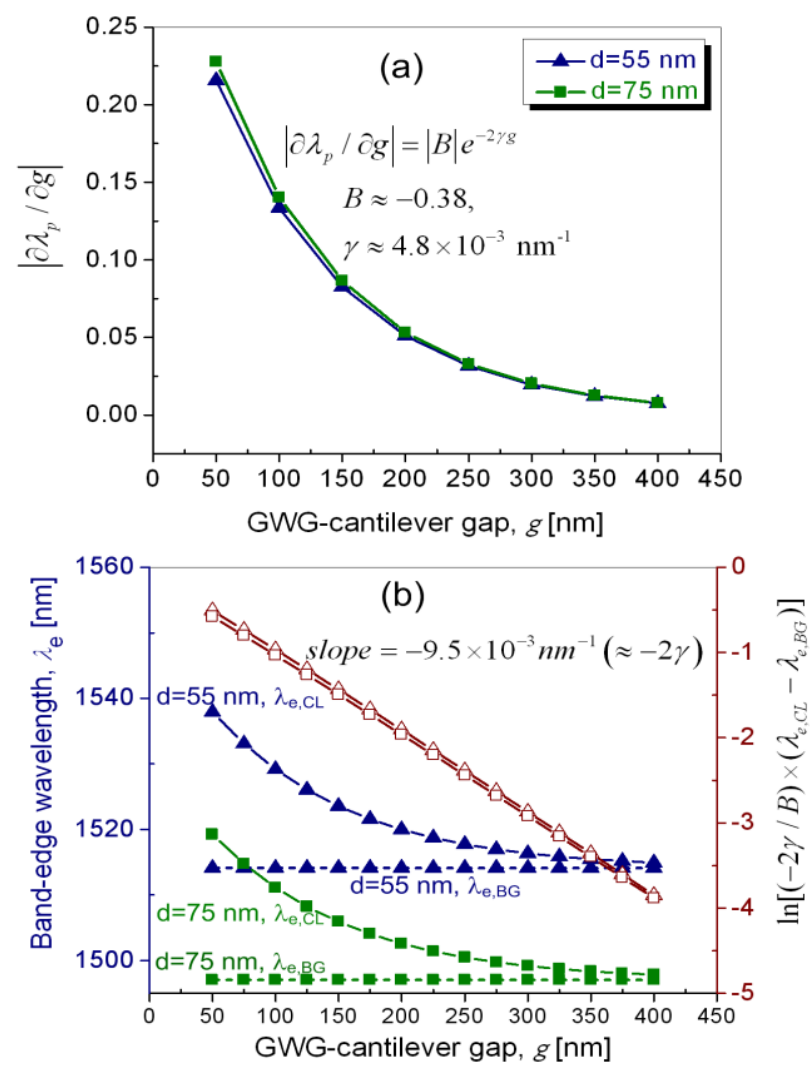

Fig. 6.4 (a) The quantity $\left|\partial \lambda_{p} / \partial g\right|$ as a function of $g$; the quantity is almost independent of the grating depth; and (b) Band-edge wavelength of the grating with and without a suspended cantilever (left-axis), and the (linear curve) $\ln \left[(-2 \gamma / B) \times\left(\lambda_{e, C L}-\lambda_{e, B G}\right)\right]$ (right-axis) for the two indicated values of the groove depth, confirming the exponential relationship presented in Eq. 6.4 (see text); 
The (wavelength dependent) sensitivity of the gas sensor, $S_{\text {gas }}$, given in Chapter 4 (Eq. 4.1), is repeated here for convenience and clarity

$$
S_{\text {gas }} \equiv\left|\left(\frac{1}{T} \frac{\partial T}{\partial C}\right)_{\lambda}\right|=\left|\left(\frac{\partial \ln T}{\partial C}\right)_{\lambda}\right|=\underbrace{\mid\left(\frac{\partial \ln T}{\partial g}\right)_{\lambda}}_{\equiv S_{M O}} \underbrace{\left|\left(\frac{\partial g}{\partial C}\right)\right|}_{\equiv S_{C L}},
$$

where $T$ is the transmittance and $C$ the gas concentration. The quantity $S_{C L}$ depends on cantilever geometry and its material properties, which include mechanical properties and properties related to gas absorption. In this work we focus on the optimization of the mechano-optical read-out, i.e., the optimization of $S_{M O}$. For simplicity we will consider only its largest value (as a function of the wavelength).

The (wavelength dependent) quantity $S_{M O}$, defined by Eq. 6.5, can be rewritten as

$$
S_{M O}=\left|\left(\frac{\partial \ln T}{\partial \lambda}\right)_{g}\left(\frac{\partial \lambda}{\partial g}\right)_{T}\right| \equiv\left|S_{\lambda}\left(\frac{\partial \lambda}{\partial g}\right)_{T}\right|,
$$

where we introduced the sensitivity for wavelength shifts, $S_{\lambda}$, and $(\partial \lambda / \partial g)_{T}$ corresponds, in regime 2 , to the quantity $\left(\partial \lambda_{p} / \partial g\right)_{\beta}$, discussed above. From BEP calculations it was found that $(\partial \lambda / \partial g)_{T}$ is nearly equal for the two regimes, so that Eq. 6.6 also holds approximately for regime 1 .

The quantity $(\partial \lambda / \partial g)_{\beta}$, which is a modal property, depends only on the gap, $g$, for a given grating and cantilever. Of the parameters to be varied in this chapter the quantity depends only slightly on the groove depth $d$ (see Fig. 6.4a) and on the cantilever thickness, $t$. As the latter will (later on; see section 6.4.3) be chosen to be fixed at $t=800 \mathrm{~nm}$, the quantity $(\partial \lambda / \partial g)_{\beta}$ (which depends only very slightly on groove depth, see Fig. 6.4b) can be left out of optimization process and we are left with optimizing the largest value (as a function of the wavelength) of

$$
S_{\lambda}=\left|\left(\frac{\partial \ln T}{\partial \lambda}\right)_{g}\right| \equiv\left|\left(\frac{\partial \ln T}{\partial \beta}\right)_{g}\left(\frac{\partial \beta}{\partial \lambda}\right)_{g}\right|,
$$


where we used that the wavelength $\lambda$ depends on the propagation constant via the dispersion curve $\beta(\lambda)$.

In equation 6.7 the factor $\partial \ln T / \partial \beta$ reflects the Fabry-Perot interferometric principle, the factor $\partial \beta / \partial \lambda$ is inversely proportional to the group velocity and so to the magnitude of the light matter interaction. Both factors influence the slope of the spectral response and may increase strongly if the resonance wavelength approaches the band edge or if the groove depth, $d$, increases (higher modal reflectance via mode mismatch and higher group index). The first effect occurs if the cantilever width increases (see Eq. 6.3). However, as we will see from the simulations presented in next section, the mode mismatch should not be too large as it is accompanied by scattering losses lowering both the $Q$ factor of the resonances and the device transmittance, $T$. A too low transmittance leads to a decrease of the device resolution (even if it has a large sensitivity) as the output signal becomes smaller or comparable to that corresponding to the dark current of the detector.

\subsection{Device optimization}

Below we will investigate the dependence of the device performance on the 5 variable parameters listed in Table 6.1, which are the grating length $(L)$, the cantilever width $(W)$ and thickness $(t)$, and the GWG-cantilever gap $(g)$. The groove depth is kept constant at $d=55 \mathrm{~nm}$ in most of the cases.

Not mentioned so far are the exact positions of the cantilever edges with respect to the grating grooves underneath them, i.e., at least some effect on modal reflection and transmission may be expected if such a cantilever edge is shifted for example from a groove to a 'tooth'. However, BEP calculations in which the edge position was shifted with steps smaller than the period did not reveal significant changes in the most relevant output parameters.

As a starting point and for completeness we will consider relevant properties of the bare grating GWG, partly published before [109]. While varying device parameters, we will mainly consider the quantity $S_{\lambda}$, from which the sensitivity can be derived using Eq. 6.6. Here we take advantage of the above observation that $(\partial \lambda / \partial g)_{\beta}$ depends only on $g$ once grating and cantilever geometry are fixed. Other parameters that will be monitored are the device transmittance (for reasons mentioned above) and the resonance peak positions for the interpretation of the results. In order to limit the 
complexity of the optimization problem a bit we assume as a criterion for optimum performance the maximum value for $S_{\lambda}$ at a gap of $g=200 \mathrm{~nm}$ and a grating depth of $d=55 \mathrm{~nm}$, with a peak transmittance not lower than (rather arbitrarily) $10^{-3}$ in the gap range $g \geq 100 \mathrm{~nm}$. In order to study the effect of the grating depth also a value of $d=75 \mathrm{~nm}$ is considered in a few cases.

\subsubsection{Bare grated waveguide}

\subsubsection{Groove depth}

The fixed, varied and considered output parameters are given in Table 6.2; results are presented in Fig. 6.5.

In Fig. 6.5a the displayed peak shift reflects the shift to lower wavelengths of the (lower wavelength) band edge on increasing groove depth. In Fig. $6.5 \mathrm{~b}$ it is shown that, in the considered parameter regime, $S_{\lambda, \max }$ growths somewhat faster than linearly with $d$. This observation also holds for results presented later for a GWG-cantilever system. The increase in $S_{\lambda}$ is related to a decreasing slope of the $\lambda$ versus $\beta$ curve for increasing groove depth, implying a shift of the resonance peak towards the band edge (see for example Fig. 6.2).

Table 6.2 Overview of input and output parameters. Blue boxed values are chosen, yellow boxed values are varied to find an optimum design.

\begin{tabular}{|l|l|l|l|}
\hline Grating & Gap & Cantilever & Output \\
\hline$L=250 \Lambda$ & \multirow{2}{*}{$g=\infty$} & $W=0$ & $\lambda_{p}$ \\
\cline { 1 - 3 }$d=55-75 \mathrm{~nm}$ & & $t=0$ & $T_{p}$ and $S_{\lambda, \max }$ \\
\hline
\end{tabular}



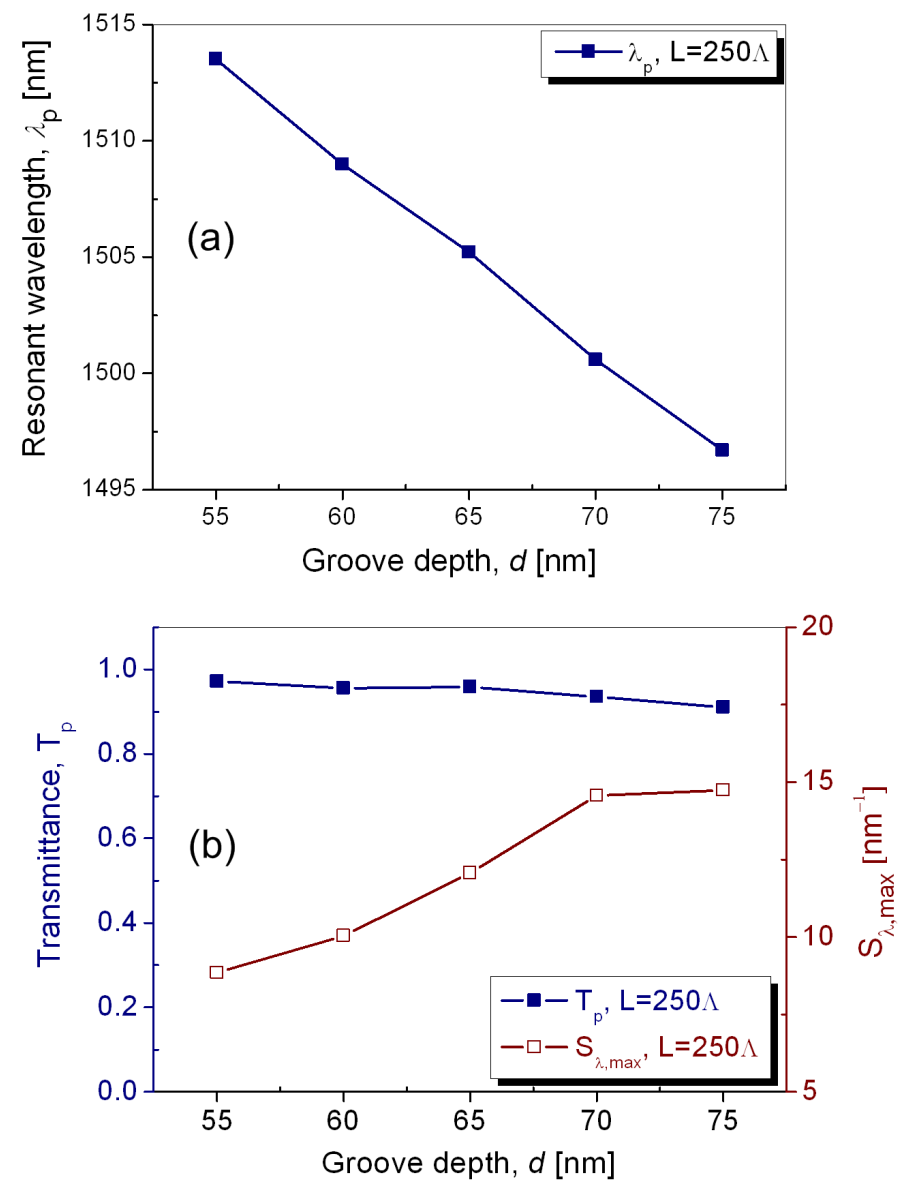

Fig. 6.5 Effect of groove depth, d, on GWG performance: (a) resonant wavelengths, and (b) transmittance at $\lambda_{p}\left(T_{p}\right)$ and maximum slope $\left(S_{\lambda, \max }\right)$ as functions of GWG groove depth. 


\subsubsection{Grating length}

We investigate the dependence of the GWG performance on its grating length $L$. The simulation parameters are listed in Table 6.3.

Table 6.3 Overview of input and output parameters. Blue boxed values are chosen, yellow boxed values are varied to find an optimum design.

\begin{tabular}{|l|l|l|l|}
\hline Grating & Gap & cantilever & Output \\
\hline$L=125 \Lambda-400 \Lambda$ & \multirow{2}{*}{$g=\infty$} & $W=0$ & $\lambda_{p}$, \\
\cline { 1 - 1 }$d=75 \mathrm{~nm}$ & & $t=0$ & $T_{p}$ and $S_{\lambda, \max }$ \\
\hline
\end{tabular}

Figure 6.6a shows the wavelength values of the considered peak as a function of GWG length, as calculated with BEP. At larger wavelengths the peaks shift towards the wavelength of the band edge, $\lambda_{e}(=1497.05 \mathrm{~nm})$. The difference $\lambda_{e}-\lambda_{p}$ is found to be approximately proportional to $1 / L^{2}$ (see Fig. 6.6b), which can easily be derived from Eq. 6.1, using that $M=L / \Lambda, \beta_{e}=\pi / \Lambda$, and assuming a parabolically shaped dispersion curve near the band edge (i.e., $\left.\lambda_{e}-\lambda \propto \sim\left(\beta-\beta_{e}\right)^{2}\right)$. The above is accompanied by a strong L-dependence of $S_{\lambda \text {,max }}$ (see Fig. 6.6c). The effect of loss due to mode mismatch is minor for the considered parameter range. 

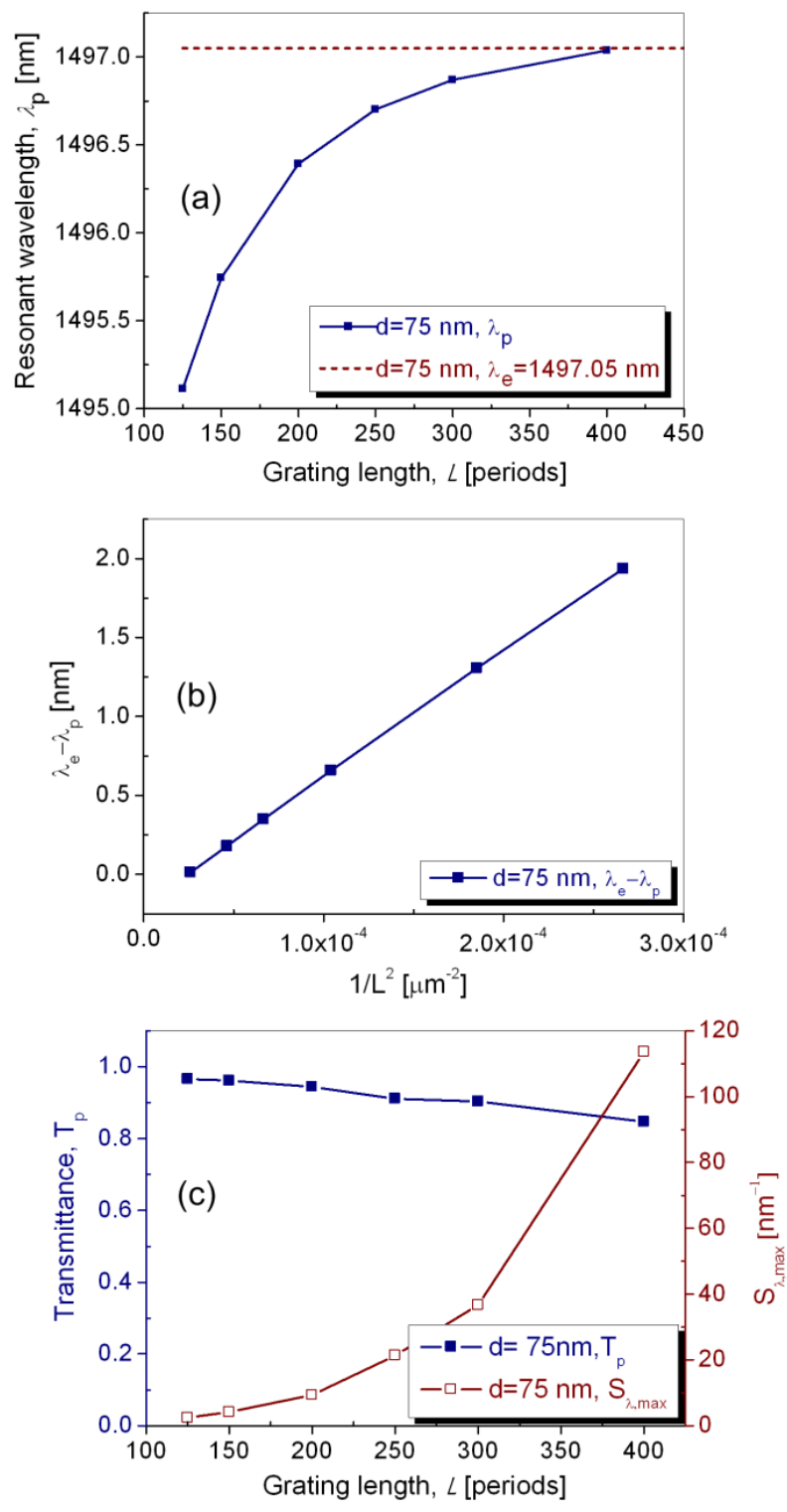

Fig. 6.6 Effect of grating length L on GWG performance: (a) resonant wavelengths as functions of the grating length, (b) difference between band edge wavelength and peak position as a function of $1 / L^{2}$ and (c) transmittance and maximum slope $S_{\lambda, \max }$ as a function of the grating length. Increasing the grating length results in a red-shift of the resonance peak towards to band-edge wavelength and an increase of $S_{\lambda, \max }$ (sensitivity) and loss. 


\subsubsection{Integrated grated waveguide with cantilever}

\subsubsection{Cantilever thickness}

The used input and output parameters are listed in Table 6.4.

Table 6.4 Overview of input and output parameters. Blue boxed values are chosen, yellow boxed values are varied to find an optimum design.

\begin{tabular}{|l|l|l|l|}
\hline Grating & Gap & cantilever & Output \\
\hline$L=250 \Lambda$ & \multirow{2}{*}{$g=200 \mathrm{~nm}, 300 \mathrm{~nm}$} & $W=0.5 \times L$ & $\lambda_{p}$, \\
\cline { 1 - 1 } & & $t$ & $T_{p}$ and $S_{\lambda, \max }$ \\
\hline
\end{tabular}

For finding an optimum value for $t$ we use as a boundary condition that $t$ has to be larger than $800 \mathrm{~nm}$ to prevent sticking of the cantilever to the substrate during fabrication (see Chapter 4). On the other hand, for mechanical bending (as a response to gas-induced stress in the receptor layer) it is of importance that the value of $t$ is chosen as small as possible (see Eq. 4.5).

In figure 6.7a the positions of the considered peak are given as a function of $t$. Starting at $\lambda_{p}=\lambda_{p, B G}$ at $t=0$ with a relatively high slope the curve saturates at $t \approx 800 \mathrm{~nm}$. It should be noted that the peak shift is proportional to the factor $(\partial \lambda / \partial g)_{\beta}$, which is of importance for the sensitivity $S_{M O}$. The graph for $S_{\lambda, \max }$, in Fig. $6.7 \mathrm{~b}$, shows that, for $g=300 \mathrm{~nm}$, this quantity has a local maximum value for $t \approx 500 \mathrm{~nm}$, and it increases for larger $t$-values. From Fig. $6.7 \mathrm{c}$, it can be seen that, for $g=200 \mathrm{~nm}, S_{\lambda, \text { max }}$ has a maximum at $t \approx 500 \mathrm{~nm}$. The lower transmittance at larger cantilever thickness, especially for $g=200 \mathrm{~nm}$, is attributed to mode mismatch, owing to a wider optical field in the cantilever region.

From the above computational results, using the boundary conditions that $t$ has to be larger than $800 \mathrm{~nm}$ to prevent sticking and that $t$ should be as small as possible (for the mechano-optical interaction) we take as an optimum value $t=800 \mathrm{~nm}$, which is assumed in all cases from now on. 

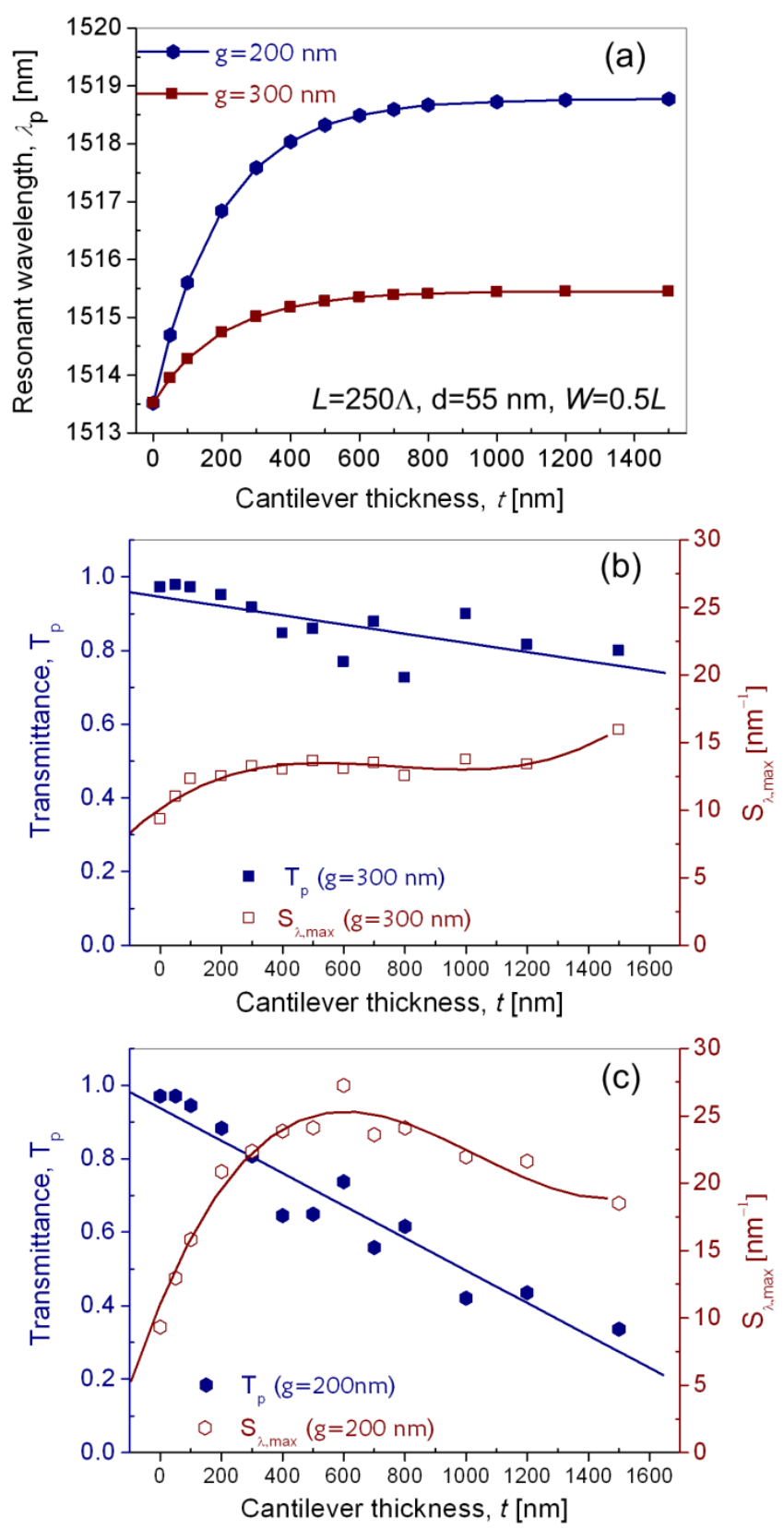

Fig. 6.7 Computational results to find an optimum value for the cantilever thickness: (a) Resonant peak position, and (b) and (c) transmittance (left-axis) and maximum slope $S_{\lambda, \max }$ (right-axis) are plotted as functions of cantilever thickness $t$ ( $t=0$ means that the device is a grating without a suspended cantilever). 


\subsubsection{Cantilever gap width}

The used input and output parameters are listed in Table 6.5.

Table 6.5 Overview of input and output parameters. Blue boxed values are chosen, yellow boxed values are varied to find an optimum design.

\begin{tabular}{|l|l|l|l|}
\hline Grating & Gap & cantilever & Output \\
\hline$L=125 \Lambda$ & \multirow{2}{*}{$g=50-400 \mathrm{~nm}$} & $W=20 \mu \mathrm{m}$ & $\partial \lambda_{p} / \partial g$, \\
& $t=800 \mathrm{~nm}$ & $T_{p}$ and $S_{\lambda, \max }$ \\
\hline
\end{tabular}

Figure 6.8a shows the wavelength position of the $1^{\text {st }}$ peak left of the band edge (left axis); also the position of the edge wavelengths of the bare grating, $\lambda_{e, B G}$, (for the two considered $d$ values) are indicated. As explained above the resonance peaks at wavelengths above $\lambda_{e, B G}$ correspond to regime 2 (see section 6.3), where $(\partial \lambda / \partial g)_{\beta}$ is assumed to be a constant (Eq. (6.4) for given $g)$. To confirm this by numerics we also plotted the quantity

$$
D \equiv \ln \left[(-2 \gamma / B) \times\left(\lambda_{p}-\lambda_{p, 1}\right)+e^{-2 \gamma g_{1}}\right]
$$

with the subscript 1 indicating the first peak value above $\lambda_{e, B G}$ (i.e., corresponding to the lowest wavelength in regime 2 , at $g=200 \mathrm{~nm}$ in both cases). Equation 6.8 follows from Eq. 6.4 and holds for regime 2 only. It then follows that (for regime 2 ) the following should hold

$$
D=-2 \gamma g
$$

which is confirmed by the straight lines of $D$ (as defined by Eq. 6.8; using parameters for $B$ and $\gamma$ given in section 6.3) versus $g$ in Fig. 6.8a and b. The slopes of these lines, both being $-8.1 \times 10^{-3} / \mathrm{nm}$ are reasonably close to $-2 \gamma\left(=-9.6 \times 10^{-3} / \mathrm{nm}\right)$.

The maximum sensitivity $S_{\lambda}$ is given in Fig. 6.8c; just as for the bare grating its value increases by a factor of $\sim 2$ in the range $d=55$ to $75 \mathrm{~nm}$. At lower gap values the transmittance drops to $\sim 0.1$ due to mode mismatch at the transitions between uncovered and cantilever-covered grating sections. 

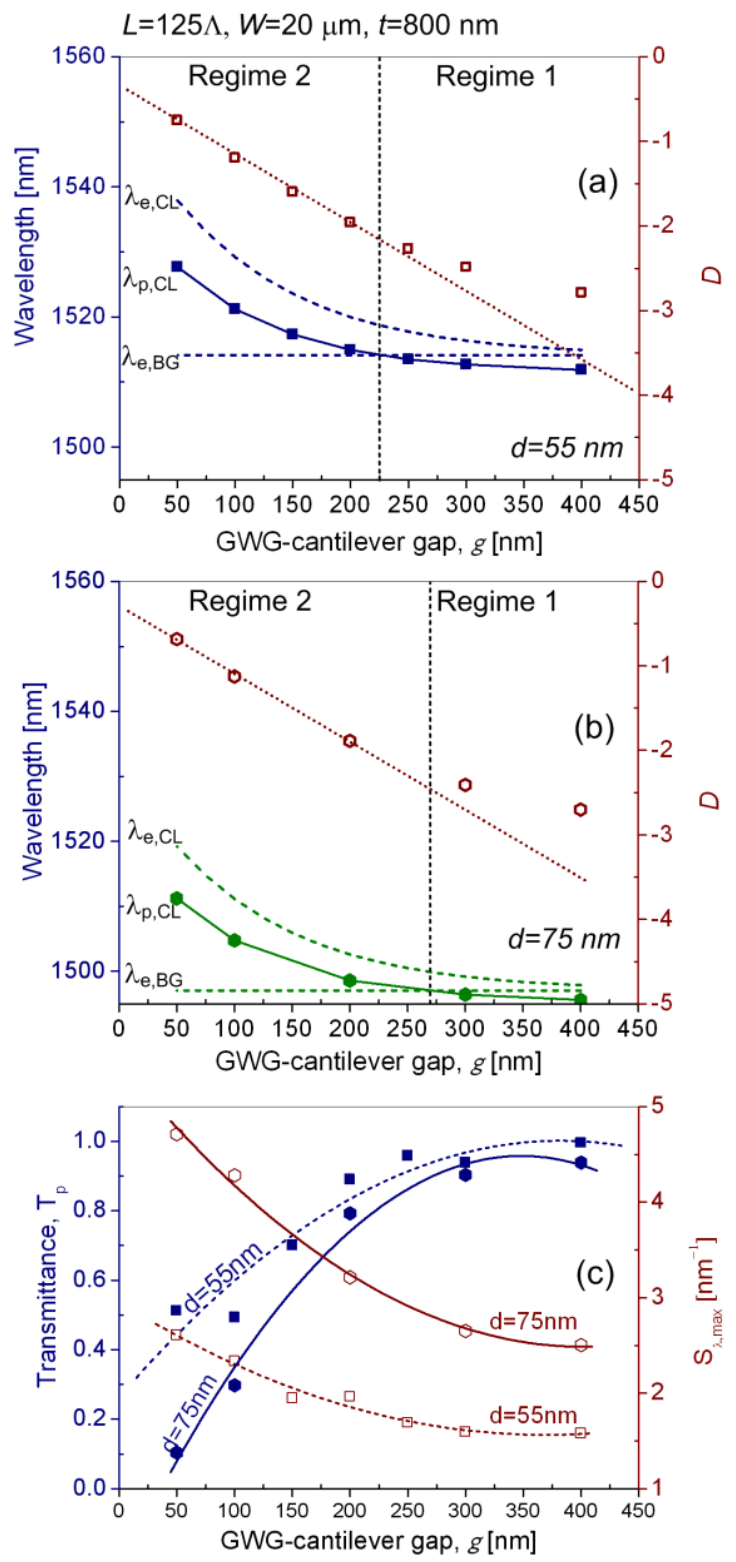

Fig. 6.8 (a) and (b) Resonant wavelengths and band-edge wavelenths (leftaxis) and the quantity $D \equiv \ln \left[(-2 \gamma / B) \times\left(\lambda_{p}-\lambda_{p, 1}\right)+e^{-2 \gamma g_{1}}\right]$ (right axis) for grating groove depth values of $d=55 \mathrm{~nm}$ and $d=75 \mathrm{~nm}$, respectively; and (c) transmittance at $\lambda_{p}\left(T_{p}\right.$ - left-axis) and maximum sensitivity $\left(S_{\lambda, \max }\right.$ - right-axis) are plotted as functions of $g$. The horizontal lines in (a) and (b) indicate the values of $\lambda_{e, B G}$ for both considered groove depths. 


\subsubsection{Ratio of cantilever width to grating length}

From our simulations we have found that the maximum value of $S_{\lambda}$ as a function of $L$ and $W$ peaks around a value of $W / L=0.5$, for which arguments will be given below. For this reason we have varied $W / L$ in our BEP simulations for three different values of the device length (see Table 6.6).

Table 6.6 Overview of input and output parameters. Blue boxed values are chosen, yellow boxed values are varied to find an optimum design.

\begin{tabular}{|l|l|l|l|}
\hline Grating & Gap & Cantilever & Output \\
\hline$L_{1-3}=125 \Lambda, 250 \Lambda, 375 \Lambda$ & \multirow{2}{*}{$g=200 \mathrm{~nm}$} & $W / L=0-1$ & $\lambda_{p}$ \\
\cline { 1 - 1 }$d=55 \mathrm{~nm}$ & & $t=800 \mathrm{~nm}$ & $T$ and $|\partial \ln T / \partial \lambda|$ \\
\hline
\end{tabular}

In figure 6.9, the resonant wavelength $\lambda_{\mathrm{p}}$ (left axis) and resonant shift $\Delta \lambda_{\mathrm{p}}$ (right axis) of the three devices are plotted as functions of the relative width $(W / L)$ of the cantilever. $W / L=0$ indicates that the system is only a bare GWC without a suspended cantilever.

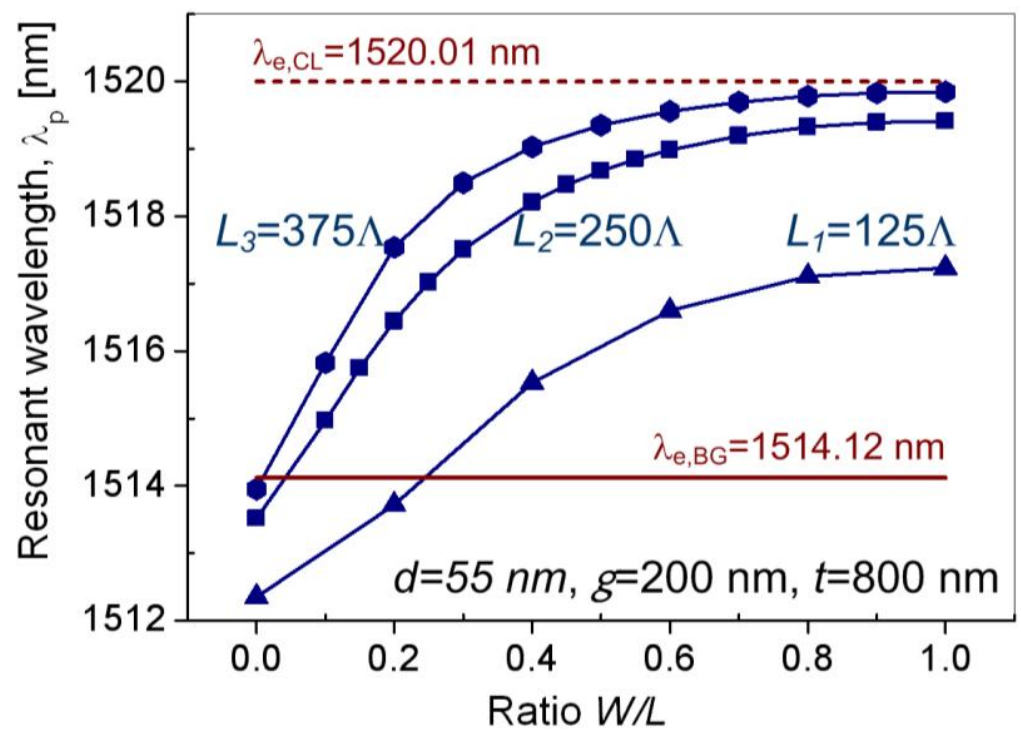

Fig. 6.9 Device performance with varied $W / L$, showing the resonant wavelength $\lambda_{p}$ as functions of cantilever relative width (W/L). The horizontal (solid) line indicating $\lambda_{e, B G}$ indicates the boundary between regime 1 (lower wavelengths) and regime 2 . 
At low values of $W / L$ the system operates in regime 1 and the peak wavelength is still far from the edge wavelength of the cantilever-covered grating $\left(\lambda_{e, C L}\right)$, implying rather low values of the group index $\left(v i a(\partial \beta / \partial \lambda)_{g}\right)$ and so of $S_{\lambda}$. For values of $W / L$ just below unity $(W / L=1$ corresponds to a fully covered GWG) the uncovered grating part is rather small, corresponding to short sections with evanescent mode propagation and so to weak modal reflections, which leads to lower values of $(\partial \ln T / \partial \beta)_{g}$ and so of $S_{\lambda}$. The above indicates that a maximum value of $S_{\lambda}$ is to be expected between the two extremes, i.e. at moderate values of $W / L$, as shown in the graphs of Fig. 6.10. 

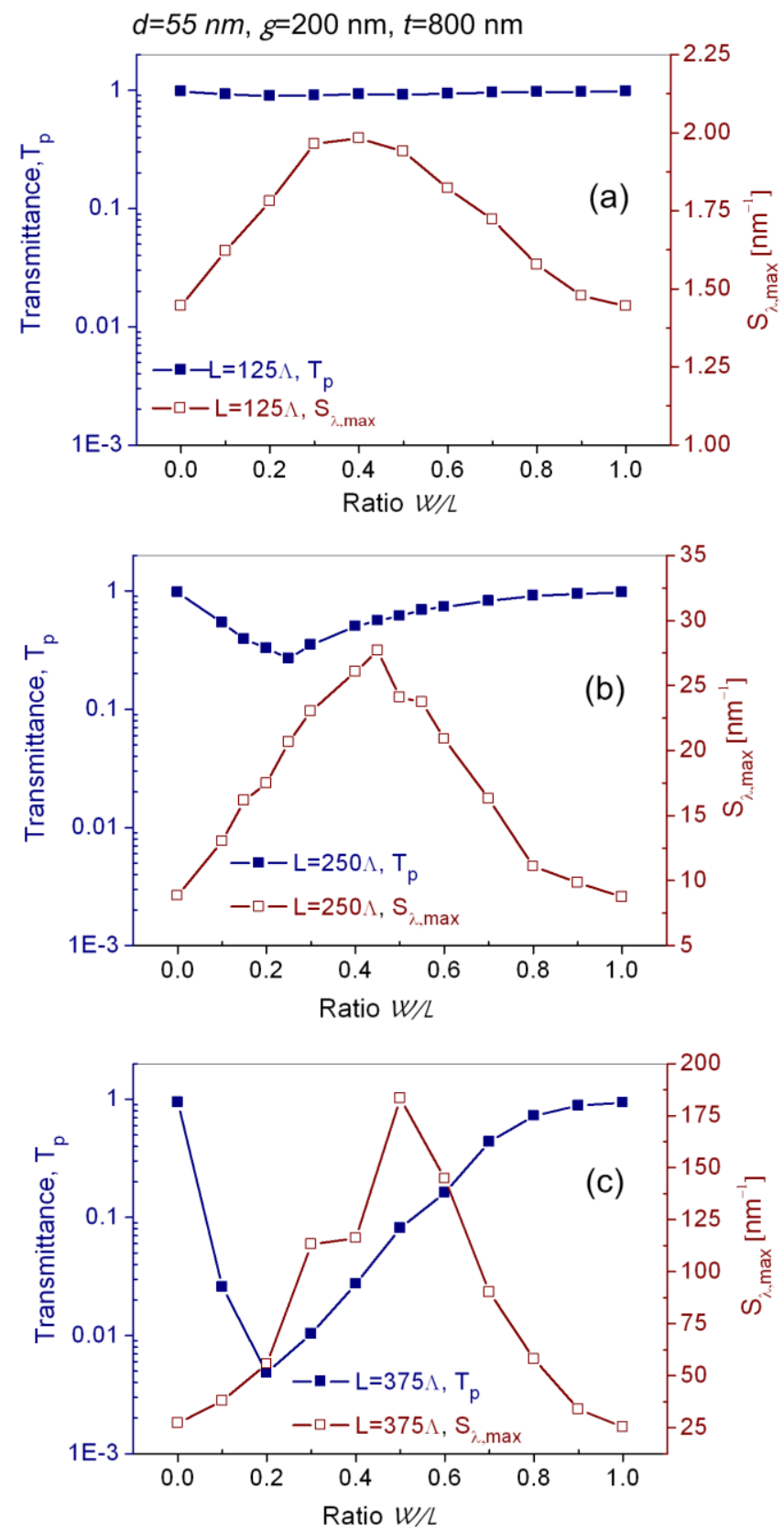

Fig. 6.10 Device performance at varying $W / L$. Transmittance at $\lambda_{p}\left(T_{p}\right.$ - leftaxis) and maximum value of $S_{\lambda}\left(S_{\lambda, \max }\right.$-right-axis) are plotted as functions of the ratio $W / L$ (cantilever width/ grating length) of devices at lengths (a) $L_{1}=125 \Lambda$, (b) $L_{2}=2501$ and (c) $L_{3}=375 \Lambda$. 
The maximum value of $S_{\lambda, \max }$ (i.e., the maximum value of $S_{\lambda}$ over a resonance peak) occurs for all three device lengths at or near $W / L=0.5$. At the latter value $S_{\lambda, \max }$ increases by a factor of almost 100 over the range $L$ $=125 \Lambda$ to $375 \Lambda$ (see Fig. 6.11).

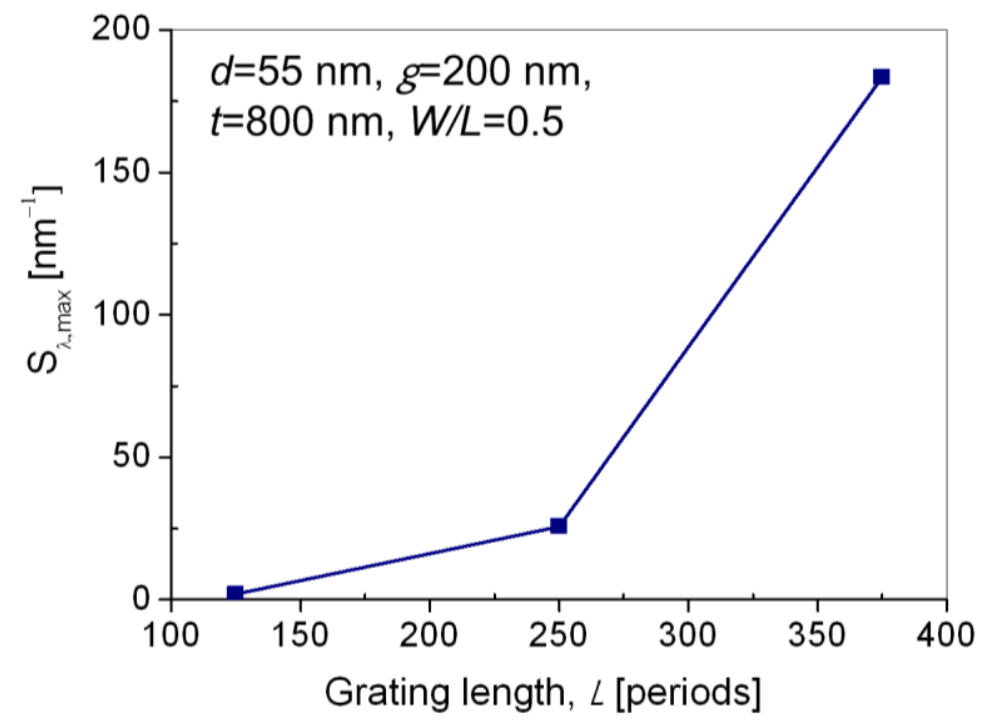

Fig. 6.11 Maximum value of $S_{\lambda}$ versus the grating length retrieved from Fig. 6.10 at $W / L=0.5$.

BEP simulations with $W / L=0.5$ and $L=375 \Lambda$ are presented in Fig. 6.12c, for $g>100 \mathrm{~nm}$. The figures $6.12 \mathrm{a}$ and $6.12 \mathrm{~b}$ are given for comparison, and to show that sensitivity as well as transmittance strongly depends on the device length.

Table 6.7 Used parameters for Fig. 6.12. Blue boxed values are fixed, yellow boxed values are varied.

\begin{tabular}{|l|l|l|l|}
\hline Grating & Gap & cantilever & Output \\
\cline { 1 - 2 }$L=125 \Lambda, 250 \Lambda, 375 \Lambda$ & \multirow{2}{*}{$g=50-400 \mathrm{~nm}$} & $W / L=0.5$ & $T_{p}$ and $S_{\lambda, \max }$ \\
\cline { 1 - 1 }$d=55 \mathrm{~nm}$ & & $t=800 \mathrm{~nm}$ & \\
\hline
\end{tabular}



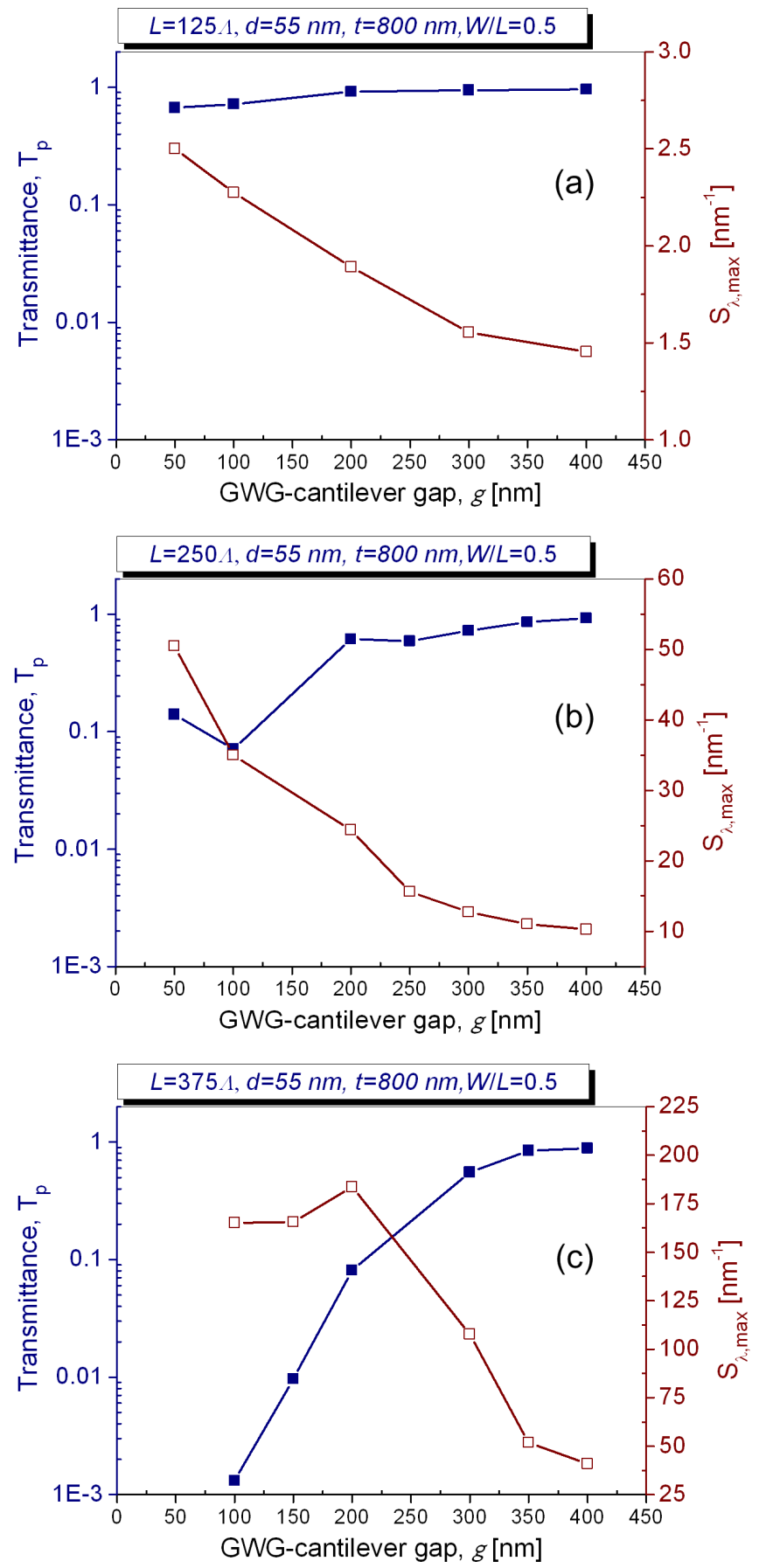

Fig. 6.12 Transmittance $T_{p}$ and $S_{\lambda, \max }$ as a function of the gap for parameters indicated in the figures. 
Taking into account the assumed boundary condition of $T_{p}>10^{-3}$ for $g>$ $100 \mathrm{~nm}$ it can be seen from Fig. 6.12c that this limit has almost been reached, implying that (with assumed other parameters) also the upper boundary of $S_{\lambda, \max }$ has been reached. This would imply a maximum value of $S_{M O}=S_{\lambda, \max }\left|(\partial \lambda / \partial g)_{\beta}\right| \approx 9.15 / \mathrm{nm}$ at $g=200 \mathrm{~nm}$, a value which is intrinsically limited (for given waveguide and GWG parameters) by the chosen device principle. In the above we have used $S_{\lambda, \max }=183 / \mathrm{nm}$ (Fig. 6.10c), and $\left|(\partial \lambda / \partial g)_{\beta}\right|=0.051$ from Fig. 6.4b. The limit of detection for changes of the gap, $L O D_{g}$, can be calculated using the theory presented in Chapter 3 (biosensing), leading to $L O D_{g}=L O D_{\lambda}\left|(\partial g / \partial \lambda)_{\beta}\right|=16 \mathrm{pm}$, where we used the experimentally obtained $L O D_{\lambda}=0.8 \mathrm{pm}$ (see Chapter 3).

\subsubsection{Summary and conclusions on device optimization}

In this section we have reported a study on the mechanism behind the observed (numerical) phenomena and on an optimization process to find trends in the relation between sensitivity and device parameters to investigate the potential of the considered sensing device. Assuming boundary conditions (mainly, transmittance $T_{p}>10^{-3}$ for $g>100 \mathrm{~nm}$ ) and chosen values of $d=55 \mathrm{~nm}$, for the groove depth, $\Lambda=490 \mathrm{~nm}$ for the grating period, and $g=200 \mathrm{~nm}$ for the gap between cantilever and GWC an optimum sensitivity was found at $t=800 \mathrm{~nm}, L \sim 375 \Lambda$ and $W=0.5 \mathrm{~L}$, for cantilever thickness, device length and cantilever width, respectively.

A few calculations performed for a groove depth of $d=75 \mathrm{~nm}$ show generally a relatively small increase, by a factor of $\sim 2$, of the sensitivity. However, at the same time the transmittance drops due to increased mode mismatch, urging the choice of a shorter device length, such that there is no or hardly any increase of the sensitivity. 


\subsection{Conclusions}

In conclusion to this chapter, we have reported on results of a numerical investigation and optimization of a novel sensing device based on the integration of a cantilever and a grated waveguide. Such a device can potentially be used for detection of small amounts of gas concentration through nanodisplacement of the suspended cantilever, which is covered by a (selective) gas absorbing layer. It is found that the sensor performance strongly depends on the grating length, the gap between cantilever and grated waveguide, and the cantilever width, and to a much lesser degree on grating depth, cantilever thickness and width. Assuming a boundary for the acceptable losses it follows that the sensitivity is intrinsically limited by the device principle for given waveguide and GWG structures. As to losses, we expect that there is still room for improvement by adapting the structure to reduce mode mismatch at waveguide to grating transitions, and also by increasing the groove depth.

The calculations have been performed via dimensionality reduction from $3 \mathrm{D}$ to $2 \mathrm{D}$, implying that scattering losses in lateral directions are ignored, leading presumably to an underestimation of the scattering losses owing to mode mismatch. For this reason it may be assumed that the here presented optimum device length should be taken a bit lower in practice, requiring experimental fine tuning. 



\section{Conclusions and outlook}

Three sensing applications utilizing the slow-light propagation and Fabry-Perot resonance in an optical grated waveguide cavity are presented in this thesis. General conclusions and an outlook on possible future research are given in this chapter. 


\subsection{Conclusions}

In the research presented in this thesis, we have focused on design, fabrication and demonstration of sensing devices based on a $\mathrm{Si}_{3} \mathrm{~N}_{4}$ grated waveguide (GWG). Three sensing applications are demonstrated, which are based on homogeneous sensing, surface sensing and mechano-optical sensing mechanisms. All three mechanisms exploit the spectral shift of the GWC transmittance induced by small changes in the environment of the GWG disturbing its modal evanescent field.

The first two mechanisms (homogeneous sensing and surface sensing) directly utilize the GWG surface, where the analytes are applied and the small changes in the homogeneous solution or the growth of an adlayer on the sensor surface are induced. These mechanisms are exploited for the bulkconcentration sensor and the label-free protein sensor. Experimentally resolutions of $6 \cdot 10^{-6}$ RIU and $\sim 4 \mathrm{pm}$ thickness growth have been obtained for bulk and surface sensing, respectively. Lower limit of detection can in principle be decreased by improving the design of the device, e.g., longer grating. However, noise analysis discussed in chapter 3 indicates that operation of the currently designed device is limited by peripheral equipment, such as the temperature control and/or inaccuracy of the scanning wavelength.

The third sensing mechanism (mechano-optical sensing) exploited small deflection changes due to the interaction between the GWC and the integrated suspended microcantilever above the GWG. The sensitivity of the set-up depends exponentially on the gap between $C L$ and $G W G$, which may limit the applicability of the sensor to a certain concentration range. The change of the gap depends on the deflection of the suspended cantilever, which can be induced by an external source (e.g., mechanical force, electrostatic force, or thermal source) or by a surface stress due to gas absorption of a receptor coated on the cantilever surface. The integrated GWG-cantilever (CL) based sensor using the mechano-optical sensing mechanism enables sensing of gases with relatively low concentrations, which is virtually impossible via the two aforementioned mechanisms. Successful fabrication and characterization for the proof of concept of the integrated GWG-CL read-out are presented in chapter 4 . The novel mechano-optical read-out scheme applied for gas sensing, which successfully detects hydrogen gas $\left(1 \% \mathrm{H}_{2}\right.$ in $\left.\mathrm{N}_{2}\right)$, is presented in chapter 5 . With the current designed 
GWG-CL device, the potential limit of detection of the cantilever bending is down to $33 \mathrm{pm}$ provided that the initial bending is well-controlled and the set-up is well temperature-stabilized. Suggestions for strong reduction of the initial bending of CLs are also discussed in chapter 5. The main goal of fabricating and demonstrating functioning devices has been fulfilled. Furthermore, numerical simulations to optimize the integrated mechanooptical read-out are presented and discussed in Chapter 6.

\subsection{Outlook}

Integrated optical sensors based on the $\mathrm{Si}_{3} \mathrm{~N}_{4}$ grated waveguides have potential for high sensitivity, simplicity and mass production. Further investigation for optimization should focus on two aspects: improvement of the device and improvement of the setup. In addition to the three sensing applications presented in this thesis, the GWG-based sensor could be investigated as to its potential for DNA sensing, where the GWG transmission spectral shift is due to the growth of an adlayer induced by the interaction between a single-stranded DNA and its corresponding probe via nucleic acid folding, i.e., adenine-thymine (A-T), guanine-cytosine ( $G-C)$.

\subsubsection{Improvement of device}

The three investigated sensing mechanisms are based on the slow-light propagation and Fabry-Perot resonance in the GWG. It can be shown that for longer GWGs the considered resonance peak sharpens up owing to the combined effect of a shift of the resonances towards the band-edge (higher group index) and a higher reflectance at the waveguide-GWG transitions. Therefore the sensitivity of the sensors can significantly be improved using longer gratings. For the mechano-optical gas sensors, cantilever parameters also need to be improved, of which the initial bending and the relative width of the cantilever (i.e., cantilever width/ grating length) are the most important.

\subsubsection{Improvement of setup}

As mentioned in chapter 3, the performance of the peripheral equipment could be improved by a better temperature stabilization (e.g., via an improved thermal isolation); the wavelength scanning could be improved by replacing it by a new updated tunable laser, or by an electro-optical tuning in combination with a narrow wavelength laser, coming from e.g., an optical 
comb laser. In addition, a (nearly) identical reference device can be incorporated in the design so that the effects of noise can be depressed via data processing. 
Appendices 


\section{A1. Process of immobilizing antibodies on $\mathrm{Si}_{3} \mathrm{~N}_{4}$ surface}

In this appendix, the process of immobilizing antibodies on the $\mathrm{Si}_{3} \mathrm{~N}_{4}$ surface, as presented in Section 3.4.3, is described in detail. The immobilization process consists of 5 steps:

\section{(1) Cleaning and activation to generate a silanol surface $(-\mathrm{Si}-\mathrm{OH})$}

The surface of the $\mathrm{Si}_{3} \mathrm{~N}_{4}$ GWC device was cleaned with the hydrofluoric acid (HF) solution (1\%) in a few minutes, and then with the $\mathrm{HNO}_{3}$ solution (100\%) in 1 hour and activated with the so-called piranha solution $\left(\mathrm{CH}_{3} \mathrm{COOH}, 100 \%: \mathrm{H}_{2} \mathrm{O}_{2}, 30 \%=4: 1\right)$ in 45 minutes. After this procedure silanol groups (- $\mathrm{Si}-\mathrm{OH})$ have been generated at the surface.

\section{(2) Silanization to generate an amino surface $\left(-\mathrm{NH}_{2}\right)$}

The GWG device with the silanol groups was next silanized in a solution containing 1 part of 3-aminopropyltriethoxysilane (APTES, 99\%) and 19 parts of ethanol (96\%). This process was quickly done in 5 seconds, after which the sample was washed 8 times with ethanol, and dried with nitrogen gas. This step results in approximately a monolayer of aminopropylsiloxane on the GWG surface. In this way reactive amino groups were introduced on the $\mathrm{Si}_{3} \mathrm{~N}_{4}$ surface.

\section{(3) Glutaraldehyde treatment to generate an aldehyde surface $(-\mathrm{CH}=\mathrm{O})$}

Glutaraldehyde was used as a bifunctional cross-linking agent to immobilize the antibodies on the chip. In this case, a mixture of a 25 wt.\% glutaric dialdehyde (Sigma-Aldrich) and PBS1x with ratio of 1:19 was used. The amino-silanized $\mathrm{Si}_{3} \mathrm{~N}_{4}$ surface was soaked in the diluted glutaraldehyde for $4 \mathrm{~h}$ at room temperature, followed by rinsing in PBS1X solution, deionized water, and drying with $\mathrm{N}_{2}$ gas. By this procedure, the amino-functional $\mathrm{Si}_{3} \mathrm{~N}_{4}$ surface was converted to a reactive surface with aldehyde groups $(-\mathrm{CH}=\mathrm{O})$ which was ready for immobilization of antibody.

\section{(4) Antibody immobilization}

PepN-antibody solution $(1 \mu \mathrm{g} / \mu \mathrm{l})$ was dissolved in PBST1x solution (PBS with Tween 20) ${ }^{6}$ with ratio of 1:19. To couple the PepN-antibody with the gutaraldehyde-treated $\mathrm{Si}_{3} \mathrm{~N}_{4}$ surface, the GWG device was kept in the

\footnotetext{
${ }^{6}$ PBST10x was obtained by adding $0.5 \mathrm{ml}$ Tween 20 to $95 \mathrm{ml}$ PBS10x.
} 
antibody solution at room temperature for $4 \mathrm{~h}$, followed by rinsing in PBST1x solution, deionized water, and drying with $\mathrm{N}_{2}$ gas.

(5) Blocking of remaining aldehyde groups

After the antibody immobilization reaction, there may remain left-over aldehyde groups. Bovine serum albumin (BSA) blocking solution was used to block any remaining aldehyde groups. The solution was made by mixing $0.2 \mathrm{~g}$ BSA, $8.793 \mathrm{ml}$ PBST1x and $1.207 \mathrm{ml}$ ethanolamine. The blocking process was carried out in $1 \mathrm{~h}$, followed by rinsing in PBST1x solution, deionized water, and drying with $\mathrm{N}_{2}$ gas. After this step, the $\mathrm{Si}_{3} \mathrm{~N}_{4}$ GWG device was ready for antigen-antibody reaction, which is described in section 3.5.4. 


\section{A2. Freeze-drying process}

In this appendix we will describe the technology steps used to release the $\mathrm{SiO}_{2} / \mathrm{Pd}$ bilayer cantilever by etching the poly-sacrificial layer underneath it, which includes the freeze-drying process, as mentioned in Chapter 4. The process is based on the standard procedure described in the MESA+ cleanroom database. Figure A2 shows the SEM images of the sticking cantilevers fabricated without using the free-drying process.
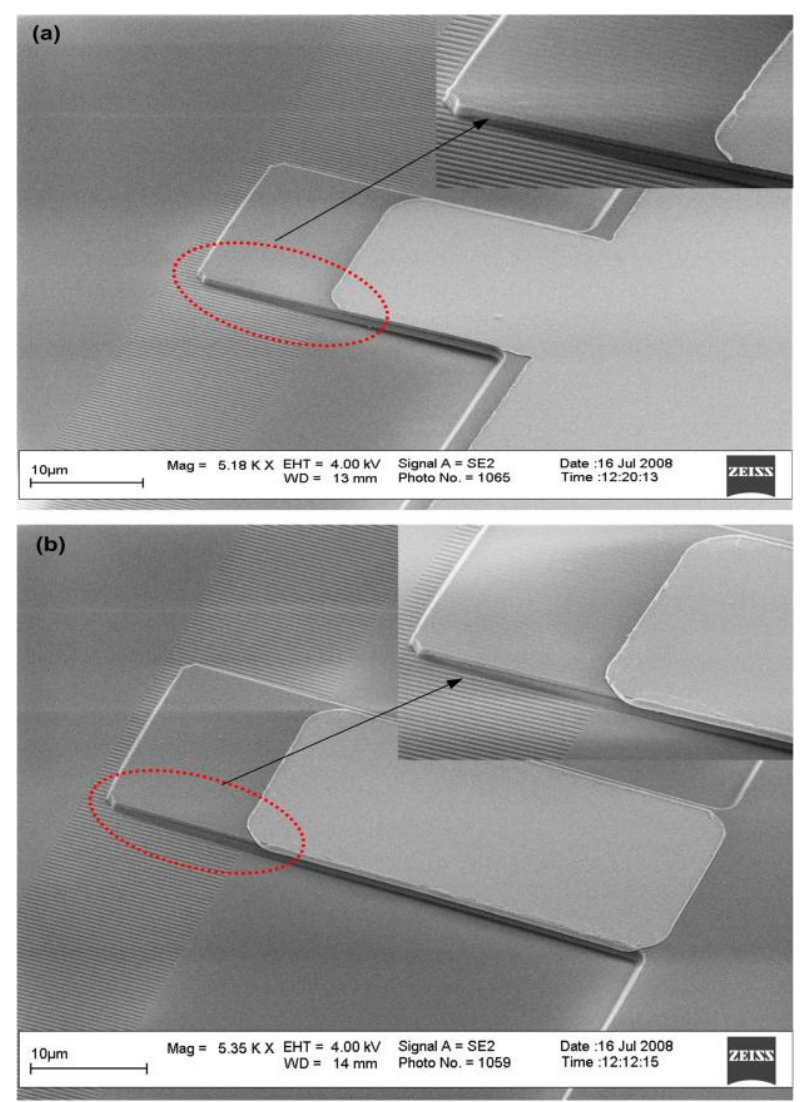

Fig. A2 Stiction of a short (a, $30 \mu \mathrm{m})$ and long (b, $50 \mu \mathrm{m})$ cantilever as released with the TMAH wet-etching process without using the freeze drying process. No stiction occurred with the $30 \mu \mathrm{m}$ long $C L$ when the freeze-drying process was used as showed in Chapter 4.

The freeze-drying setup mainly consists of a Peltier chuck and a glass hood, which together form a reactor (chamber). The Peltier chuck is connected with a thermo-couple, power supply and water cooling system. 
The glass hood is connected with a $\mathrm{N}_{2}$ flow supply. Before starting the freeze-drying process, the reaction chamber is rinsed with an $\mathrm{N}_{2}$ flow (0.5 bar, room temperature). After the poly-Si sacrificial layer is completely etched by the solution TMAH, $5 \%$ (see Chapter 4), the freeze-drying process is applied, which consists of the following steps:

1. Rinse TMAH chemicals $(200 \mathrm{ml}$ ) in the etching beaker (say beaker 1 , where the sample has been etched) using a dilute step method: remove $100 \mathrm{ml}$ of the chemical and add $100 \mathrm{ml}$ dilute ionized (DI) water, wait 5 minutes and remove $100 \mathrm{ml}$ of the diluted chemical. This step is repeated $\sim 10$ times to have all TMAH chemicals completely replaced by DI water.

2. Dilute DI water in beaker 1 with $500 \mathrm{ml}$ IPA (isopropyl alcohol), using the step method 5 times.

3. Transfer the sample with the liquid (IPA +DI water) on top of it, to a beaker containing $200 \mathrm{ml}$ IPA (beaker 2), and soak it for $\sim 150$ minutes. The steps 2 and 3 aim to completely replace DI water by IPA.

4. Put the sample into a beaker containing $200 \mathrm{ml}$ cyclohexane (beaker 3) for $\sim 30$ minutes.

5. Put the sample into a second cyclohexane beaker (200ml, beaker 4) for $\sim 30$ minutes. The steps 5 and 6 aim to replace IPA completely by cyclohexane.

6. Take the sample out of the beaker 4 and put it on a clean room tissue paper to remove the cyclohexane from the backside of the sample. (Otherwise, ice - on the backside, occurring during next step - may disturb the thermal contact between the sample and the chuck).

7. Transfer the sample to the chuck, set at $-5^{\circ} \mathrm{C}$, wait for a few seconds until ice formed on the sample before closing the glass hood. (The freezing point of cyclohexane is at $6.55^{\circ} \mathrm{C}$ ).

8. Wait for $\sim 10$ minutes until it is seen that the ice is gone from the top of the sample. Wait for another $\sim 30$ minutes to remove the ice underneath the released $\mathrm{CL}$.

9. Heat the reactor to room temperature ( $\sim 15$ minutes) to avoid moisture condensation on the wafer and keep rinsing with the $\mathrm{N}_{2}$ flow.

10. Check the result using an interference microscope. 


\section{A3. Dry-eching process}

As discussed in Chapter 4 - Section 4.5.2, using the $S_{6}$ plasma dry-etching for our purpose resulted in poor quality devices with high surface roughness. Here we describe (for possible use in the future) the main features of the dryetching process.

For $\mathrm{SF}_{6}$ plasma dry-etching, an extra resist mask is required to protect unetched areas. As illustrated in Fig. A3, holes (1) are made on the resist mask so that the $\mathrm{SF}_{6}$ plasma stream can attack and remove the poly-Si sacrificial layer underneath the resist mask areas (2). However, not only the poly-Si layer (green) but also the $\mathrm{Si}_{3} \mathrm{~N}_{4}$ layer underneath it (yellow) is affected removed with different etch depths depending on the direction of the plasma stream (Fig. A.3a), which results in an ill-defined surface (Fig. A3b, and see more Fig 4.6 - Chapter 4).

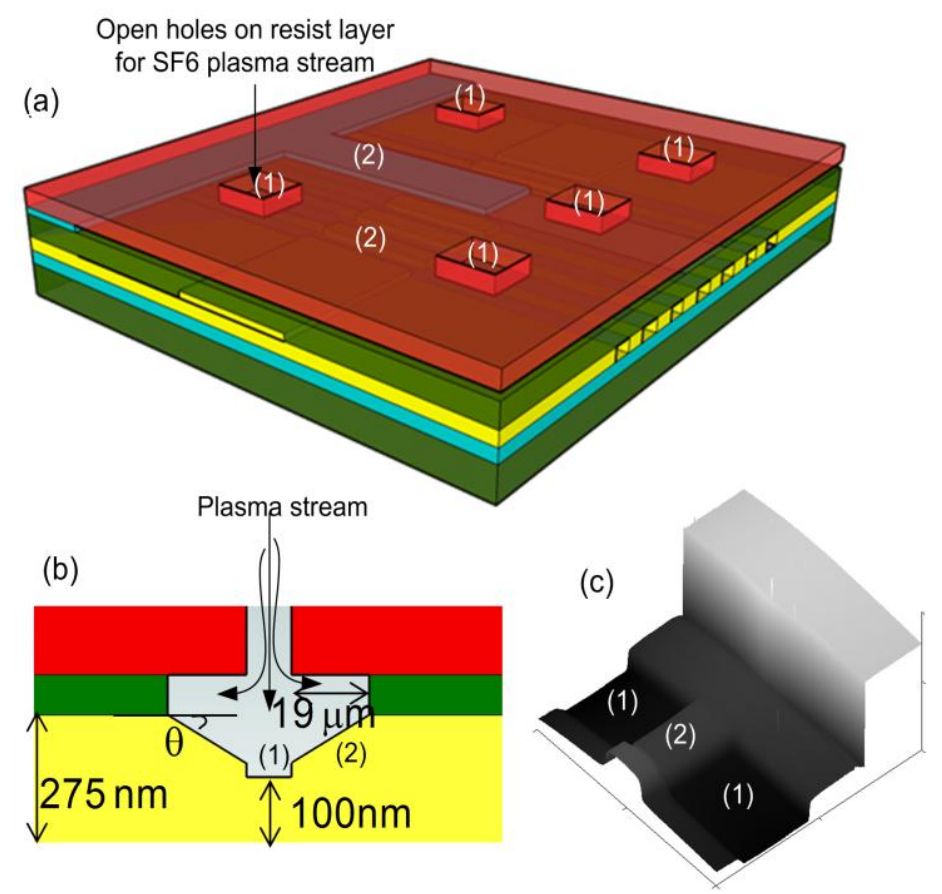

Fig. A3 (a) Schematic of etching the poly-Si sacrificial layer (dark green) underneath the cantilever: (1) holes for plasma stream and (2) areas need to be etched, expectedly poly-Si only, (b) schematic of etched profile which is hard to control, i.e., over etch on $\mathrm{Si}_{3} \mathrm{~N}_{4}$ layer (yellow), and (c) AFM image of the profile shown in (b). 


\section{A4. Technology steps for the fabrication of the GWG-CL device}

The table below contains the technology steps for the fabrication of GWG-CL devices discussed in Chapter 4.

\begin{tabular}{|c|c|c|}
\hline Step & Process description & Mask \\
\hline 1 & Substrate selection - Silicon $<100>$ OSP & \\
\hline 2 & Standard cleaning & \\
\hline 3 & $\mathrm{Si}_{3} \mathrm{~N}_{4}$ stoichiometric LPCVD & \\
\hline 4 & Standard cleaning & \\
\hline 5 & Lithography - Olin 907-12: ridge WG patterning & 1 \\
\hline 6 & BHF (1:7) wet etching of $\mathrm{Si}_{3} \mathrm{~N}_{4}$ : ridge $\mathrm{WG}$ formation & \\
\hline 7 & RIE alignment key & \\
\hline 8 & Resist stripping by oxygen plasma & \\
\hline 9 & Standard cleaning & \\
\hline 10 & BARC DUV30 spin-coating CL2000 & \\
\hline 11 & MA-N2403 spin-coating CL2000 & \\
\hline 12 & AZ-aquatar spin-coating CL2000 & \\
\hline 13 & LIL exposure (without mask) & \\
\hline 14 & UV exposure (with mask ): define grating area & 2 \\
\hline 15 & Development 75\% OPD & \\
\hline 16 & RIE $\mathrm{Si}_{3} \mathrm{~N}_{4}$ gratings: transfer grating pattern to $\mathrm{Si}_{3} \mathrm{~N}_{4}$ & \\
\hline 17 & Standard cleaning & \\
\hline 18 & Poly-Si LPCVD: sacrificial layer deposition & \\
\hline 19 & Standard cleaning & \\
\hline 20 & Lithography - Olin 907-12: anchor patterning & 3 \\
\hline 21 & RIE poly-Si (Etske): anchor formation & \\
\hline 22 & Resist stripping by oxygen plasma & \\
\hline 23 & Standard cleaning & \\
\hline 24 & TEOS $\mathrm{SiO}_{2}$ LPCVD & \\
\hline 25 & $\mathrm{PECVD} \mathrm{SiO}_{2}$ (if applicable) & \\
\hline 26 & Standard cleaning & \\
\hline 27 & Lithography - Olin 907-12: $\mathrm{SiO}_{2}$ cantilever patterning & 4 \\
\hline 28 & $\mathrm{RIE} \mathrm{SiO}_{2}: \mathrm{SiO}_{2}$ cantilever formation & \\
\hline 29 & Resist stripping by oxygen plasma & \\
\hline 30 & Standard cleaning & \\
\hline 31 & Lithography - Olin 907-12: receptor layer patterning & 5 \\
\hline 32 & Sputering $\mathrm{Cr} 10 \mathrm{~nm}$ & \\
\hline 33 & Sputtering Pd (30)/50 nm & \\
\hline 34 & Lift-off Olin-PR in Acetone & \\
\hline
\end{tabular}




\begin{tabular}{|c|l|c|}
\hline 35 & Lithography - Olin 908-35: dicing-line patterning & 6 \\
\hline 36 & Deep RIE etching: dicing-line etching & \\
\hline 37 & Resist coating - Olin 907-17: for dicing protection & \\
\hline $38 \mathrm{a}$ & Dicing Silicon & \\
\hline $39 \mathrm{a}$ & $\mathrm{TMAH} 5 \%, 70^{\circ} \mathrm{C}$ wet-etching & \\
\hline 40a & Freeze-drying & \\
\hline $38 \mathrm{~b}$ & Lithography for patterning the resist mask & \\
\hline $39 \mathrm{~b}$ & $\mathrm{SF}_{6}$ plasma etching & \\
\hline 40b & Dicing Silicon & \\
\hline
\end{tabular}




\section{Publications}

\section{Peer-reviewed journal articles}

1. S. V. Pham, René M. de Ridder, and H. J. W. M. Hoekstra, "Numerical investigation and optimization of a mechano-optical sensor based on an optical grated-waveguide cavity," in preparation.

2. So V. Pham, Meindert Dijkstra, Anton J. F. Hollink, Lasse J. Kauppinen, René M. de Ridder, Markus Pollnau, Paul V. Lambeck and Hugo J. W. M. Hoekstra, "On-chip bulk-index concentration and direct, label-free protein sensing utilizing an optical grated-waveguide cavity," (submitted 2012).

3. S. V. Pham, M. Dijkstra, H. A. G. M. van Wolferen, M. Pollnau, G. J. M. Krijnen, and H. J. W. M. Hoekstra, "Integrated mechano-optical hydrogen-gas sensor using cantilever bending read-out with a $\mathrm{Si}_{3} \mathrm{~N}_{4}$ grated waveguide," Optics Letters, Vol. 36, Issue 15, pp. 3003-3005, 2011.

4. S. V. Pham, L. J. Kauppinen, M. Dijkstra, H. A. G. M. van Wolferen, R. M. de Ridder, and H. J. W. M. Hoekstra, "Read-out of cantilever bending with a grated waveguide optical cavity," Photonics Technology Letters, IEEE, vol.23, no.4, pp.215-217, 2011.

5. M. D. Nguyen, H. Nazeer, K. Karakaya, S. V. Pham, R. Steenwelle, M. Dekkers, L. Abelmann, D. H. A. Blank and G. Rijnders, Characterization of epitaxial $\mathrm{Pb}(\mathrm{Zr}, \mathrm{Ti}) \mathrm{O}_{3}$ thin films deposited by pulsed laser deposition on silicon cantilevers, J. Micromech. Microeng.20 085022, Issue 8, 2010.

6. Pham Van So, Jangkwen-Lee, Sanghun-Shin and Jaichan-Lee, "Electromechanical Performance of Piezoelectric Actuators in Inkjet Print Head”, Integrated Ferroelectrics, 98: 251-258, 2008.

\section{Conference presentations/papers}

1. S. V. Pham, M. Dijkstra, A. J. F. Hollink, R. M. de Ridder, G. J. M. Krijnen, Paul V. Lambeck M. Pollnau, and H. J. W. M. Hoekstra, "Grated waveguide optical cavity for sensing," Poster presentation, Proc. 16th European Conference on Integrated Optics (ECIO), April 18-20, 2012, Sitges/Barcelona, Spain. Paper 114. 
2. S. V. Pham, M. Dijkstra, H. A. G. M. van Wolferen, M. Pollnau, G. J. M. Krijnen, and H. J.W. M. Hoekstra, "A novel mechano-optical sensor based on read-out with a $\mathrm{Si}_{3} \mathrm{~N}_{4}$ grated waveguide," Proceedings of the 2011 Annual Symposium of the IEEE Photonics Benelux Chapter, 1-2 December 2011, Ghent, Belgium. pp. 6164, Department of Information Technology, Ghent University.

3. S. V. Pham, M. Dijkstra, A. J. F. Hollink, R. M. de Ridder, H. A. G. M. van Wolferen, G. J. M. Krijnen, M. Pollnau, H. J. W. M. Hoekstra, "Grated waveguide cavity for label-free protein and mechano-optical gas sensing," Oral presentation, The 2011 IQEC / CLEO Pacific Rim Conference, August 28 - September 1, 2011, Sydney, Australia, paper 177.

4. S. V. Pham, M. Dijkstra, A. J. F. Hollink, R. M. de Ridder, M. Pollnau, and $\mathrm{H}$. J. W. M. Hoekstra, " $\mathrm{Si}_{3} \mathrm{~N}_{4}$ grated waveguide optical cavity based sensors for bulk-index concentration, label-free protein, and mechano-optical gas sensing," Oral presentation (invited talk), The twentieth annual International Laser Physics Workshop (LPHYS'11), July 11- 15, 2011, Sarajevo, Bosnia and Herzegovina.

5. M. Pollnau, B. I. Akca, N. Ismail, C. Dongre, S. V. Pham, K. Wörhoff, R. M. de Ridder, and H.J.W.M. Hoekstra, "Biophotonic sensors on a microchip for trace-gas detection, DNA and enzyme analysis, Raman spectroscopy, and optical coherence tomography", Joint Annual Meeting of the Swiss Physical Society and Austrian Physical Society, Lausanne, Switzerland, in Bulletin SPG/SSP, Vol. 28, 2011, paper 213.

6. S. V. Pham, M. Dijkstra, A.J.F. Hollink, R.M. de Ridder, M. Pollnau, and H.J.W.M. Hoekstra, "Applications of a Grated Waveguide Optical Cavity for Label-free Protein and Gas Sensing," Oral presentation, Applied Nanophotonics (ANP) retreat, May 16-17, 2011, Ootmarsum, the Netherlands.

7. S. V. Pham, M. Dijkstra, A. J. F. Hollink, R. M. de Ridder, M. Pollnau, and H. J. W. M. Hoekstra, "Compact Integrated Optical Sensors based on a $\mathrm{Si}_{3} \mathrm{~N}_{4}$ Grated Waveguide Optical Cavity," Oral presentation, The European Conference on Lasers and Electro-Optics and the XIIth European Quantum Electronics Conference (CLEO®/Europe-EQEC 2011), May 22-26, 2011, Munich, Germany, paper CK2.1.

8. S. V. Pham, M. Dijkstra, H. A. G. M. van Wolferen, M. Pollnau, G. J. M. Krijnen and H. J. W. M. Hoekstra, "A Novel Mechano-Optical Sensor based on Read-out with a $\mathrm{Si}_{3} \mathrm{~N}_{4}$ Grated Waveguide," Oral presentation, Conference on Lasers and Electro-Optics (CLEO), May 1- 


\section{6, 2011, Baltimore, Maryland, USA, paper CThQ4.}

9. S. V. Pham, M. Dijkstra, A.J.F. Hollink, L.J. Kauppinen, R.M. de Ridder and H.J.W.M. Hoekstra, "Label-free Enzyme Sensing with a $\mathrm{Si}_{3} \mathrm{~N}_{4}$ Grated Waveguide Optical Cavity," Poster presentation, Conference on Lasers and Electro-Optics (CLEO), May 1-6, 2011, Baltimore, Maryland, USA, paper JWA108.

10. S. V. Pham, L.J. Kauppinen, R.M. de Ridder, H.J.W.M. Hoekstra, "Numerical investigation and optimization of a mechano-optical sensor based on a grated waveguide optical cavity", Oral presentation, Proc. 2010 Symp. IEEE Photonics Benelux Chapter, November 18-19, 2010, Delft, The Netherlands, pp. 145-148, ISBN 978-90-78314-15-8.

11. S. V. Pham, L.J. Kauppinen, M. Dijkstra, H.A.G.M. van Wolferen, R.M. de Ridder and H.J.W.M.Hoekstra, "Read-out of Cantilever Bending with a Grated Waveguide Optical Cavity", Poster presentation, MESA+ Annual Meeting, September 14, 2010, Enschede, the Netherlands.

12. S. V. Pham, L.J. Kauppinen, M. Dijkstra, H.A.G.M. van Wolferen, R.M. de Ridder and H.J.W.M.Hoekstra, "Read-out of Cantilever Bending with a Grated Waveguide Optical Cavity", Applied Nanophotonics (ANP) internal workshop, May 27-28, 2010, Bad Bentheim, Germany.

13. S. V. Pham, L.J. Kauppinen, M. Dijkstra, H.A.G.M. van Wolferen, R.M. de Ridder and H.J.W.M.Hoekstra, "Cantilever deflection readout with a grated waveguide optical cavity", Oral presentation, Proc. 15th European Conference on Integrated Optics (ECIO), April 7-9, 2010, Cambridge, UK, paper ThE6.

14. S. V. Pham, L.J. Kauppinen, M. Dijkstra, H.A.G.M. van Wolferen, R.M. de Ridder and H.J.W.M. Hoekstra, "Mechano-optical sensors for hydrogen gas: Fabrication and Proof of concept", Poster presentation, The Sense of Contact 12, April 8, 2010, Woudschoten, Zeist, The Netherlands.

15. S. V. Pham, L.J. Kauppinen, M. Dijkstra, H.A.G.M. van Wolferen, R.M. de Ridder and H.J.W.M.Hoekstra, "Fabrication of mechanooptical sensors for hydrogen gas”, Poster presentation, Annual Workshop on Semiconductor Advances for Future Electronics and Sensors (SAFE), November 26-27, 2009, Veldhoven, the Netherlands, [BEST POSTER AWARD]. 
16. L. J. Kauppinen, C. Dongre, M. Hoekman, S. V. Pham, M. Dijkstra, M. Pollnau, R. M. de Ridder and H. J. W. M. Hoekstra, "Advanced Photonic Devices for (multi) sensing of Biomolecules and Gases," MESA+ Annual Meeting, September 21, 2009, Enschede, the Netherlands.

17. S. V. Pham, L. J. Kauppinen, M. Dijkstra, H. A. G. M. van Wolferen, R. M. de Ridder and H. J. W. M. Hoekstra, "Simulation, design and fabrication of microcantilver-based 10 sensors for the highly sensitive detection of gas concentrations", Invited talk at Laboratory for Nanotechnology, Vietnam National University of Ho Chi Minh City, August 18, 2009, Ho Chi Minh City, Vietnam.

18. S. V. Pham, L. J. Kauppinen, M. Dijkstra, H. A. G. M. van Wolferen, R. M. de Ridder and H. J. W. M. Hoekstra, "Photonic crystal waveguides integrated with microcantilevers as a novel platform for gas sensors",Oral presentation, the 7th International Symposium on Modern Optics and Its Applications (ISMOA), August 10-14, 2009, Bandung, Indonesia.

19. S. V. Pham, L. J. Kauppinen, M. Dijkstra, H. A. G. M. van Wolferen, R. M. de Ridder and H. J. W. M. Hoekstra, "Simulation and fabrication of a mechano-optical sensor for nano-displacements", Oral presentation, 1st Nano Today Conference, August 2-5, 2009, Singapore.

20. Pham Van So, L. J. Kauppinen, H. J. W. M. Hoekstra, M. Dijkstra, H. A. G. M. van Wolferen, G. J. M. Krijnen and R. M. de Ridder, "Fabrication of microcantilever-based 10 grated waveguide sensors for detection of nano-displacements", Poster presentation, Proc. Annual Symposium of the IEEE/LEOS Benelux Chapter, November 2728, 2008, Enschede, The Netherlands [BEST POSTER AWARD].

21 Pham Van So, L. J. Kauppinen, H. J. W. M. Hoekstra, M. Dijkstra, H. A. G. M. van Wolferen, G. J. M. Krijnen and R. M. de Ridder, "Simulation and fabrication of microcantilever-based 10 grated waveguide sensors for detection of nano-displacements: primary results”, Poster presentation, MESA+ Annual Meeting, September 23, 2008, Enschede, The Netherlands. 


\section{References}

[1] M. J. Madou, Fundamentals of microfabrication: the science of miniaturization. Boca Raton: CRC Press, 2002.

[2] B. Haab, "Methods and applications of antibody microarrays in cancer research," Proteomics, vol. 3, pp. 2116-2122, 2003.

[3] J. Gooding, "Biosensor technology for detecting biological warfare agents: Recent progress and future trends," Analytica Chimica Acta, vol. 559, pp. 137-151, 2006.

[4] P. S. Waggoner and H. G. Craighead, "Micro- and nanomechanical sensors for environmental, chemical, and biological detection," Lab on a Chip, vol. 7, pp. 1238-1255, 2007.

[5] M. Dennison and A. Turner, "Biosensors for Environmental Monitoring," Biotechnology Advances, vol. 13, pp. 1-12, 1995.

[6] J. Daniels and N. Pourmand, "Label-free impedance biosensors: Opportunities and challenges," Electroanalysis, vol. 19, pp. 1239-1257, 2007.

[7] G. Voirin and R. Kunz, "Optical sensor using an immunological reaction and a fluorescent marker," Google Patents, 2001.

[8] J. Heng, A. Aksimentiev, C. Ho, P. Marks, Y. Grinkova, S. Sligar, K. Schulten, and G. Timp, "The electromechanics of DNA in a synthetic nanopore," Biophysical Journal, vol. 90, pp. 1098-1106, 2006.

[9] A. Aksimentiev, "Deciphering ionic current signatures of DNA transport through a nanopore," Nanoscale, vol. 2, pp. 468-483, 2010.

[10] F. Patolsky, G. Zheng, O. Hayden, M. Lakadamyali, X. Zhuang, and C. Lieber, "Electrical detection of single viruses," Proceedings of the National Academy of Sciences of the United States of America, vol. 101, pp. 14017-14022, 2004.

[11] F. Ishikawa, H. Chang, M. Curreli, H. Liao, C. Olson, P. Chen, R. Zhang, R. Roberts, R. Sun, R. Cote, M. Thompson, and C. Zhou, "Label-Free, Electrical Detection of the SARS Virus N-Protein with Nanowire Biosensors Utilizing Antibody Mimics as Capture Probes," Acs Nano, vol. 3, pp. 1219-1224, 2009.

[12] T. Burg, M. Godin, S. Knudsen, W. Shen, G. Carlson, J. Foster, K. Babcock, and S. Manalis, "Weighing of biomolecules, single cells and single nanoparticles in fluid," Nature, vol. 446, pp. 1066-1069, 2007.

[13] R. Datar, S. Kim, S. Jeon, P. Hesketh, S. Manalis, A. Boisen, and T. Thundat, "Cantilever Sensors: Nanomechanical Tools for Diagnostics," Mrs Bulletin, vol. 34, pp. 449-454, 2009. 
[14] G. Wu, R. Datar, K. Hansen, T. Thundat, R. Cote, and A. Majumdar, "Bioassay of prostate-specific antigen (PSA) using microcantilevers," Nature Biotechnology, vol. 19, pp. 856-860, 2001.

[15] C. O'Sullivan and G. Guilbault, "Commercial quartz crystal microbalances - theory and applications," Biosensors \& Bioelectronics, vol. 14, pp. 663-670, 1999.

[16] M. Iqbal, M. Gleeson, B. Spaugh, F. Tybor, W. Gunn, M. Hochberg, T. Baehr-Jones, R. Bailey, and L. Gunn, "Label-Free Biosensor Arrays Based on Silicon Ring Resonators and High-Speed Optical Scanning Instrumentation," IEEE Journal of Selected Topics in Quantum Electronics, vol. 16, pp. 654-661, 2010.

[17] H. K. Hunt and A. M. Armani, "Label-free biological and chemical sensors," Nanoscale, vol. 2, pp. 1544-1559, 2010.

[18] X. Fan, I. White, S. Shopoua, H. Zhu, J. Suter, and Y. Sun, "Sensitive optical biosensors for unlabeled targets: A review," Analytica Chimica Acta, vol. 620, pp. 8-26, 2008.

[19] P. V. Lambeck, "Integrated optical sensors for the chemical domain," Measurement Science \& Technology, vol. 17, pp. R93-R116, 2006.

[20] R. Kunz, "Miniature integrated optical modules for chemical and biochemical sensing1," Sensors and Actuators B: Chemical, vol. 38, pp. 13-28, 1997.

[21] C. Chao and L. Guo, "Design and optimization of microring resonators in biochemical sensing applications," Journal of Lightwave Technology, vol. 24, pp. 1395-1402, 2006.

[22] M. Estevez, M. Alvarez, and L. Lechuga, "Integrated optical devices for lab-on-a-chip biosensing applications," Laser \& Photonics Reviews, 2011.

[23] J. Van Gent, P. V. Lambeck, H. J. M. Kreuwel, G. J. Gerritsma, E. J. R. Sudholter, D. N. Reinhoudt, and T. J. A. Popma, "Optimization of a Chemooptical Surface-Plasmon Resonance Based Sensor," Applied Optics, vol. 29, pp. 2843-2849, 1990.

[24] J. Van Gent, P. V. Lambeck, R. J. Bakker, T. J. A. Popma, E. J. R. Sudholter, and D. N. Reinhoudt, "Design and Realization of a SurfacePlasmon Resonance-Based Chemo-Optical Sensor," Sensors and Actuators a-Physical, vol. 26, pp. 449-452, 1991.

[25] T. J. Yen and Y. C. Lai, "A plasmonic biosensor demonstrates high sensitivity and long-distance detection," in SPIE Newsroom, 2011.

[26] J. Homola, S. S. Yee, and G. Gauglitz, "Surface plasmon resonance sensors: review," Sensors and Actuators B: Chemical, vol. 54, pp. 3 15, 1999. 
[27] M. L. Gorodetsky, A. A. Savchenkov, and V. S. Ilchenko, "Ultimate Q of optical microsphere resonators," Optics Letters, vol. 21, pp. $453-$ 455, 1996.

[28] S. Arnold, M. Khoshsima, I. Teraoka, S. Holler, and F. Vollmer, "Shift of whispering-gallery modes in microspheres by protein adsorption," Optics Letters, vol. 28, pp. 272-274, 2003.

[29] N. M. Hanumegowda, I. M. White, C. J. Stica, B. C. Patel, H. Oveys, and $\mathrm{X}$. Fan, "Development of label-free microsphere optical resonator bio/chemical sensors," Proc. of SPIE, Vol. 6004, pp. 60040I-1, 2005.

[30] M. Hossein-Zadeh and K. J. Vahala, "Free ultra-high-Q microtoroid: a tool for designing photonic devices," Optics Express, vol. 15, pp. 166 175, 2007.

[31] D. K. Armani, T. J. Kippenberg, S. M. Spillane, and K. J. Vahala, "Ultra-high-Q toroid microcavity on a chip," Nature, vol. 421, pp. 925-928, 2003.

[32] C. Chao and L. Guo, "Biochemical sensors based on polymer microrings with sharp asymmetrical resonance," Applied Physics Letters, vol. 83, pp. 1527-1529, 2003.

[33] C. Barrios, M. Banuls, V. Gonzalez-Pedro, K. Gylfason, B. Sanchez, A. Griol, A. Maquieira, H. Sohlstrom, M. Holgado, and R. Casquel, "Label-free optical biosensing with slot-waveguides," Optics Letters, vol. 33, pp. 708-710, 2008.

[34] J. Hu, X. Sun, A. Agarwal, and L. Kimerling, "Design guidelines for optical resonator biochemical sensors," Journal of the Optical Society of America B-Optical Physics, vol. 26, pp. 1032-1041, 2009.

[35] M. R. Lee and P. M. Fauchet, "Two-dimensional silicon photonic crystal based biosensing platform for protein detection," Opt. Express, vol. 15, pp. 4530-4535, 2007.

[36] V. Toccafondo, J. García-Rupérez, M. Bañuls, A. Griol, J. Castelló, S. Peransi-Llopis, and A. Maquieira, "Single-strand DNA detection using a planar photonic-crystal-waveguide-based sensor," Optics Letters, vol. 35, pp. 3673-3675, 2010.

[37] S. Mandal, J. M. Goddard, and D. Erickson, "A multiplexed optofluidic biomolecular sensor for low mass detection," Lab Chip, vol. 9, pp. 2924-2932, 2009.

[38] L. Kauppinen, H. Hoekstra, and R. de Ridder, "A compact refractometric sensor based on grated silicon photonic wires," Sensors and Actuators B-Chemical, vol. 139, pp. 194-198, 2009. 
[39] M. W. Sigrist, "Trace gas monitoring by laser photoacoustic spectroscopy and related techniques (plenary)," Review of Scientific Instruments, vol. 74, pp. 486-490, 2003.

[40] C. Eranna, B. C. Joshi, D. P. Runthala, and R. P. Gupta, "Oxide materials for development of integrated gas sensors - A comprehensive review," Critical Reviews in Solid State and Materials Sciences, vol. 29, pp. 111-188, 2004.

[41] E. Rossinyol, A. Prim, E. Pellicer, J. Rodriguez, F. Peiro, A. Cornet, J. R. Morante, B. Z. Tian, T. Bo, and D. Y. Zhao, "Mesostructured pure and copper-catalyzed tungsten oxide for NO2 detection," Sensors and Actuators B-Chemical, vol. 126, pp. 18-23, 2007.

[42] Z. Zhao, M. A. Carpenter, H. Xia, and D. Welch, "All-optical hydrogen sensor based on a high alloy content palladium thin film," Sensors and Actuators B-Chemical, vol. 113, pp. 532-538, 2006.

[43] Z. Y. Hu, T. Thundat, and R. J. Warmack, "Investigation of adsorption and absorption-induced stresses using microcantilever sensors," Journal of Applied Physics, vol. 90, pp. 427-431, 2001.

[44] Britton, Jones, Oden, Hu, Warmack, Smith, Bryan, and Rochelle, Multiple-input microcantilever sensors vol. 82, 2000.

[45] S. T. Hekkert, M. J. Staal, R. H. M. Nabben, H. Zuckermann, S. Persijn, L. J. Stal, L. A. C. J. Voesenek, F. J. M. Harren, J. Reuss, and D. H. Parker, "Laser photoacoustic trace gas detection, an extremely sensitive technique applied in biological research," Instrumentation Science \& Technology, vol. 26, pp. 157-175, 1998.

[46] N. Fabricius, G. Gauglitz, and J. Ingenhoff, "A Gas Sensor Based on an Integrated Optical Mach-Zehnder Interferometer," Sensors and Actuators B-Chemical, vol. 7, pp. 672-676, 1992.

[47] N. Cioffi, D. de Ceglia, M. De Sario, A. D’Orazio, V. Petruzzelli, F. Prudenzano, M. Scalora, S. Trevisi, and M. Vincenti, "MetalloDielectric Multilayer Sensor for Hydrogen Detection in Exhaled Breath." Proc. 13th European Conference on Integrated Optics (ECIO), 25-27 April 2007, Copenhagen, Denmark.

[48] C. D. Fernandez, M. G. Manera, J. Spadavecchia, G. Maggioni, A. Quaranta, G. Mattei, M. Bazzan, E. Cattaruzza, M. Bonafini, E. Negro, A. Vomiero, S. Carturan, C. Scian, G. Della Mea, R. Rella, L. Vasanelli, and P. Mazzoldi, "Study of the gas optical sensing properties of Au-polyimide nanocomposite films prepared by ion implantation," Sensors and Actuators B-Chemical, vol. 111, pp. 225229, 2005. 
[49] P. Li and X. X. Li, "A single-sided micromachined piezoresistive $\mathrm{SiO} 2$ cantilever sensor for ultra-sensitive detection of gaseous chemicals," Journal of Micromechanics and Microengineering, vol. 16, pp. 2539 2546, 2006.

[50] J. Thaysen, A. Boisen, O. Hansen, and S. Bouwstra, "Atomic force microscopy probe with piezoresistive read-out and a highly symmetrical Wheatstone bridge arrangement," Sensors and Actuators a-Physical, vol. 83, pp. 47-53, 2000.

[51] J. Fritz, M. K. Baller, H. P. Lang, H. Rothuizen, P. Vettiger, E. Meyer, H. J. Guntherodt, C. Gerber, and J. K. Gimzewski, "Translating biomolecular recognition into nanomechanics," Science, vol. 288, pp. 316-318, 2000.

[52] L. G. Carrascosa, M. Moreno, M. Álvarez, and L. M. Lechuga, "Nanomechanical biosensors: a new sensing tool," TrAC Trends in Analytical Chemistry, vol. 25, pp. 196-206, 2006.

[53] B. Ilic, H. G. Craighead, S. Krylov, W. Senaratne, C. Ober, and P. Neuzil, "Attogram detection using nanoelectromechanical oscillators," Journal of Applied Physics, vol. 95, pp. 3694-3703, Apr 12004.

[54] D. R. Baselt, B. Fruhberger, E. Klaassen, S. Cemalovic, C. L. Britton Jr, S. V. Patel, T. E. Mlsna, D. McCorkle, and B. Warmack, "Design and performance of a microcantilever-based hydrogen sensor," Sensors and Actuators B: Chemical, vol. 88, pp. 120-131, 2003.

[55] A. Loui, F. T. Goericke, T. V. Ratto, J. Lee, B. R. Hart, and W. P. King, "The effect of piezoresistive microcantilever geometry on cantilever sensitivity during surface stress chemical sensing," Sensors and Actuators A: Physical, vol. 147, pp. 516-521, 2008.

[56] S. Okuyama, Y. Mitobe, K. Okuyama, and K. Matsushita, "Hydrogen Gas Sensing Using a Pd-Coated Cantilever," Japanese Journal of Applied Physics, vol. 39, p. 3584.

[57] E. Lee, J. M. Lee, J. H. Koo, W. Lee, and T. Lee, "Hysteresis behavior of electrical resistance in $\mathrm{Pd}$ thin films during the process of absorption and desorption of hydrogen gas," International journal of hydrogen energy, vol. 35, pp. 6984-6991, 2010.

[58] E. M. Larsson, B. Kasemo, and C. Langhammer, "Localized surface plasmons shed light on nanoscale metal hydrides," Advanced Materials, vol. 22, pp. 4628-4633, 2010.

[59] W. C. L. Hopman, P. Pottier, D. Yudistira, J. van Lith, P. V. Lambeck, R. M. De La Rue, A. Driessen, H. J. W. M. Hoekstra, and R. M. de Ridder, "Quasi-one-dimensional photonic crystal as a compact 
building-block for refractometric optical sensors," Selected Topics in Quantum Electronics, IEEE Journal of, vol. 11, pp. 11-16, 2005.

[60] W. C. L. Hopman, H. J. W. M. Hoekstra, R. Dekker, L. Zhuang, and R. M. de Ridder, "Far-field scattering microscopy applied to analysis of slow light, power enhancement, and delay times in uniform Bragg waveguide gratings," Opt. Express, vol. 15, pp. 1851-1870, 2007.

[61] Wikipedia, "http://en.wikipedia.org/wiki/Slow_light."

[62] L. Hau, S. Harris, Z. Dutton, and C. Behroozi, "Light speed reduction to 17 metres per second in an ultracold atomic gas," Nature, vol. 397, pp. 594-598, 1999.

[63] M. Kash, V. Sautenkov, A. Zibrov, L. Hollberg, G. Welch, M. Lukin, Y. Rostovtsev, E. Fry, and M. Scully, "Ultraslow group velocity and enhanced nonlinear optical effects in a coherently driven hot atomic gas," Physical review letters, vol. 82, pp. 5229-5232, 1999.

[64] D. Budker, D. Kimball, S. Rochester, and V. Yashchuk, "Nonlinear magneto-optics and reduced group velocity of light in atomic vapor with slow ground state relaxation," Physical review letters, vol. 83, 1999.

[65] M. Bigelow, N. Lepeshkin, and R. Boyd, "Observation of ultraslow light propagation in a ruby crystal at room temperature," Physical Review Letters, vol. 90, pp. 113903, 2003.

[66] M. Povinelli, S. Johnson, and J. Joannopoulos, "Slow-light, bandedge waveguides for tunable time delays," Optics Express, vol. 13, pp. 7145-7159, 2005.

[67] J. Garcia, P. Sanchis, A. Martinez, and J. Marti, "1D periodic structures for slow-wave induced non-linearity enhancement," Optics Express, vol. 16, pp. 3146-3160, 2008.

[68] T. Baba, "Slow light in photonic crystals," Nature Photonics, vol. 2, pp. 465-473, 2008.

[69] T. Krauss, "Slow light in photonic crystal waveguides," Journal of Physics D-Applied Physics, vol. 40, pp. 2666-2670, 2007.

[70] A. Canciamilla, M. Torregiani, C. Ferrari, F. Morichetti, R. De la Rue, A. Samarelli, M. Sorel, and A. Melloni, "Silicon coupled-ring resonator structures for slow light applications: potential, impairments and ultimate limits," Journal of Optics, vol. 12, 2010.

[71] J. Y. Lee and P. M. Fauchet, "Slow-light dispersion in periodically patterned silicon microring resonators," Optics Letters, vol. 37, pp. 58-60, 2012.

[72] J. Ctyroky, S. Helfert, R. Pregla, P. Bienstman, R. Baets, R. De Ridder, R. Stoffer, G. Klaasse, J. Petracek, P. Lalanne, J. Hugonin, and R. De 
La Rue, "Bragg waveguide grating as a 1D photonic band gap structure: COST 268 modelling task," Optical and Quantum Electronics, vol. 34, pp. 455-470, 2002.

[73] J. Bendickson, J. Dowling, and M. Scalora, "Analytic expressions for the electromagnetic mode density in finite, one-dimensional, photonic band-gap structures," Physical Review E, vol. 53, pp. 41074121, 1996.

[74] A. Yariv, "Coupled-mode theory for guided-wave optics," leee Journal of Quantum Electronics, vol. QE 9, pp. 919-933, 1973.

[75] K. Winick, "Effective-index method and coupled-mode theory for almost-periodic waveguide gratings: a comparison," Applied Optics, vol. 31, pp. 757-764, 1992.

[76] H. Hoekstra, W. Hopman, J. Kautz, R. Dekker, and R. De Rider, "A simple coupled mode model for near band-edge phenomena in grated waveguides," Optical and Quantum Electronics, vol. 38, pp. 799-813, 2006.

[77] P. S. J. Russell, "Optics of Floquet-Bloch waves in dielectric gratings," Applied Physics B: Lasers and Optics, vol. 39, pp. 231-246, 1986.

[78] T. Yu, L. Wang, and J. He, "Bloch wave formalism of photon lifetime in distributed feedback lasers," Journal of the Optical Society of America B-Optical Physics, vol. 26, pp. 1780-1788, 2009.

[79] M. Gnan, G. Bellanca, H. Chong, P. Bassi, and R. De La Rue, "Modelling of Photonic Wire Bragg Gratings," Optical and Quantum Electronics, vol. 38, pp. 133-148, 2006.

[80] J. Ctyroky, J. Homola, P. V. Lambeck, S. Musa, H. J. W. M. Hoekstra, R. D. Harris, J. S. Wilkinson, B. Usievich, and N. M. Lyndin, "Theory and modelling of optical waveguide sensors utilising surface plasmon resonance," Sensors and Actuators B-Chemical, vol. 54, pp. 66-73, 1999.

[81] P. V. Lambeck, H. J. W. M. Hoekstra, J. van Lith, and G. van Elzakker, "Two novel integrated optical sensor types for measuring chemical concentrations, based on chemically induced changes of modal field profiles," Journal of Nonlinear Optical Physics \&amp; Materials, vol. 13, pp. 209-227, 2004.

[82] R. E. Kunz and J. Dübendorfer, "Miniature integrated-optical wavelength analyzer chip," Opt. Lett., vol. 20, pp. 2300-2302, 1995.

[83] M. Wiki and R. E. Kunz, "Wavelength-interrogated optical sensor for biochemical applications," Opt. Lett., vol. 25, pp. 463-465, 2000. 
[84] J. H. Berends, G. J. Veldhuis, P. V. Lambeck, and T. J. A. Popma, "Device Equivalence in Integrated-Optics," Journal of Lightwave Technology, vol. 13, pp. 2082-2086, 1995.

[85] W. F. A. Engbers, "Adiabatic slow light excitation in grated channel waveguides." vol. Master thesis: University of Twente, 2005.

[86] M. Wiki, R. Kunz, G. Voirin, K. Tiefenthaler, and A. Bernard, "Novel integrated optical sensor based on a grating coupler triplet1," Biosensors and Bioelectronics, vol. 13, pp. 1181-1185, 1998.

[87] M. Wiki, H. Gao, M. Juvet, and R. Kunz, "Compact integrated optical sensor system," Biosensors and Bioelectronics, vol. 16, pp. 37-45, 2001.

[88] M. Wiki and R. Kunz, "Wavelength-interrogated optical sensor for biochemical applications," Optics letters, vol. 25, pp. 463-465, 2000.

[89] S. V. Pham, L. J. Kauppinen, M. Dijkstra, H. A. G. M. van Wolferen, R. M. de Ridder, and H. J.W. M. Hoekstra, "Read-Out of Cantilever Bending With a Grated Waveguide Optical Cavity," IEEE Photonics Technology Letters, pp. 215-217, 2011.

[90] S. V. Pham, M. Dijkstra, H. A. G. M. van Wolferen, M. Pollnau, G. J. M. Krijnen, and H. J. W. M. Hoekstra, "Integrated mechano-optical hydrogen-gas sensor using cantilever bending read-out with a Si3N4 grated waveguide," Optics Letters, vol. 36, pp. 3003-3005, 2011.

[91] H. Hoekstra, P. Lambeck, H. Uranus, and T. Koster, "Relation between noise and resolution in integrated optical refractometric sensing," Sensors and Actuators B-Chemical, vol. 134, pp. 702-710, 2008.

[92] J. D. Joannopoulos, S. G. Johnson, J. N. Winn, and R. D. Meade, Photonic Crystals: Molding the Flow of Light. Princeton, New Jersey: Princeton University Press, 2008.

[93] P. Yeh, "Electromagnetic propagation in birefringent layered media," Journal of the Optical Society of America, vol. 69, pp. 742-756, 1979.

[94] C. Dongre, M. Pollnau, and H. Hoekstra, "All-numerical noise filtering of fluorescence signals for achieving ultra-low limit of detection in biomedical applications," Analyst, vol. 136, pp. 1248-1251, 2011.

[95] C2V, "http://www.c2v.nl."

[96] D. J. Segelstein, "The complex refractive index of water," University of Missouri-Kansas City, 1981.

[97] "Longwood University, www.longwood.edu/staff/buckalewdw/Immlec/lmm14.ppt." 
[98] T. Sakata, M. Kamahori, and Y. Miyahara, "Immobilization of oligonucleotide probes on Si3N4 surface and its application to genetic field effect transistor," Materials Science \& Engineering C-Biomimetic and Supramolecular Systems, vol. 24, pp. 827-832, 2004.

[99] G. Yu and S. Yen, "Hydrogen ion diffusion coefficient of silicon nitride thin films," Applied Surface Science, vol. 202, pp. 68-72, 2002.

[100] S. Jamasb, S. Collins, and R. Smith, "A physical model for threshold voltage instability in $\mathrm{Si}_{3} \mathrm{~N}_{4}$-Gate $\mathrm{H}^{+}$-sensitive FET's (pH ISFET's)," IEEE Transactions on Electron Devices, vol. 45, pp. 1239-1245, 1998.

[101] M. Esashi and T. Matsuo, "Integrated Micro Multi lon Sensor Using Field Effect of Semiconductor," IEEE Transactions on Biomedical Engineering, vol. 25, pp. 184-192, 1978.

[102] " IMEnz Bioengineering BV, L Zielstraweg 1, 9713 GX, Groningen The Netherlands."

[103] F. Chavagnat, M. Casey, and J. Meyer, "Purification, characterization, gene cloning, sequencing, and overexpression of aminopeptidase $\mathrm{N}$ from Streptococcus thermophilus A," Applied and Environmental Microbiology, vol. 65, pp. 3001-3007, 1999.

[104] L. J. Kauppinen, M. Dijkstra, H. J. W. M. Hoekstra, and R. M. de Ridder, "Label-free Enzyme Sensing with Grated Silicon Photonic Wire," in Annual Symposium of the IEEE Photonics Benelux Chapter, Brussels, Belgium, 2009, pp. 41-44.

[105] M. Banuls, V. Gonzalez-Pedro, C. Barrios, R. Puchades, and A. Maquieira, "Selective chemical modification of silicon nitride/silicon oxide nanostructures to develop label-free biosensors," Biosensors \& Bioelectronics, vol. 25, pp. 1460-1466, 2010.

[106] B. Sutapun, M. Tabib-Azar, and A. Kazemi, "Pd-coated elastooptic fiber optic Bragg grating sensors for multiplexed hydrogen sensing," Sensors and Actuators B: Chemical, vol. 60, pp. 27-34, 1999.

[107] S. Okuyama, Y. Mitobe, K. Okuyama, and K. Matsushita, "Hydrogen Gas Sensing Using a Pd-Coated Cantilever," Japanese Journal of Applied Physics, vol. 39, p. 3584, 2000.

[108] D. lannuzzi, M. Slaman, J. H. Rector, H. Schreuders, S. Deladi, and M. C. Elwenspoek, "A fiber-top cantilever for hydrogen detection," Sensors and Actuators B: Chemical, vol. 121, pp. 706-708, 2007.

[109] L. J. Kauppinen, H. J. W. M. Hoekstra, M. Dijkstra, R. M. d. Ridder, and G. J. M. Krijnen, "Grated waveguide optical cavity as a compact sensor for sub-nanometre cantilever deflections," Proc. 14th European Conference on Integrated Optics (ECIO), 11-13 June 2008, Eindhoven, The Netherlands. 
[110] W. C. Young and R. G. Budynas, Roark's formulas for stress and strain, 7th ed.: McGraw-Hill, 2002.

[111] Y.-l. Chou, H.-C. Chiang, and C.-C. Wang, "Study on Pd functionalization of microcantilever for hydrogen detection promotion," Sensors and Actuators B: Chemical, vol. 129, pp. 72-78, 2008.

[112] M. D. Nguyen, H. Nazeer, K. Karakaya, S. V. Pham, R. Steenwelle, M. Dekkers, L. Abelmann, D. H. A. Blank, and G. Rijnders, "Characterization of epitaxial $\mathrm{Pb}$ ( $\mathrm{Zr}, \mathrm{Ti}) \mathrm{O} 3$ thin films deposited by pulsed laser deposition on silicon cantilevers," Journal of Micromechanics and Microengineering, vol. 20, p. 085022, 2010.

[113] M. Calleja, J. Tamayo, M. Nordstrom, and A. Boisen, "Low-noise polymeric nanomechanical biosensors," Applied Physics Letters, vol. 88, pp. 113901-1-13901-3, 2006.

[114] J. M. Gere and S. P. Timoshenko, Mechanics of Materials, 4th SI ed.: Stanley Thornes, 1999.

[115] A. Boisen, S. Dohn, S. S. Keller, S. Schmid, and M. Tenje, "Cantileverlike micromechanical sensors," Reports on Progress in Physics, vol. 74, p. 036101, 2010.

[116] W. Benecke and $W$. Riethmuller, "Applications of silicon microactuators based on bimorph structures," 1989, pp. 116-120.

[117] J. Matovic, J. Lamovec, and Z. Djinovic, "Bimaterial infrared detector with efficient suppression of interference from ambient temperature," vol. 1, pp. 349-352, 2004.

[118] W. Riethmuller and W. Benecke, "Thermally excited silicon microactuators," Electron Devices, IEEE Transactions on, vol. 35, pp. 758-763, 1988.

[119] H. Choi and J. Park, "Prediction of residual stress distribution in multistacked thin film by curvature measurement and iterative FEA," Journal of Mechanical Science and Technology, vol. 19, pp. 10651071, 2005.

[120] C. Mastrangelo, "Adhesion-related failure mechanisms in micromechanical devices," Tribology Letters, vol. 3, pp. 223-238, 1997.

[121] Niels Tas, Tonny Sonnenberg, Henri Jansen, Rob Legtenberg, and Miko Elwenspoek, "Stiction in surface micromachining," Journal of Micromechanics and Microengineering, vol. 6, p. 385, 1996.

[122] R. P. a. I. D. W. W Merlijn van Spengen, "A physical model to predict stiction in MEMS," Journal of Micromechanics and Microengineering, vol. 12, p. 702, 2002. 
[123] A. Witvrouw, H. A. C. Tilmans, and I. De Wolf, "Materials issues in the processing, the operation and the reliability of MEMS," Microelectronic Engineering, vol. 76, pp. 245-257, 2004.

[124] E. Forsén, Z. J. Davis, M. Dong, S. G. Nilsson, L. Montelius, and A. Boisen, "Dry release of suspended nanostructures," Microelectronic Engineering, vol. 73-74, pp. 487-490, 2004.

[125] Y. Yee, M. Park, and K. Chun, "A sticking model of suspended polysilicon microstructure including residual stress gradient and postrelease temperature," Journal of Microelectromechanical Systems, vol. 7, pp. 339-344, 1998.

[126] "Intellisuite software, http://www.intellisense.com/."

[127] G. L. Weibel and C. K. Ober, "An overview of supercritical CO2 applications in microelectronics processing," Microelectronic Engineering, vol. 65, pp. 145-152, 2003.

[128] "MESA+ Institute for Nanotechnology, http://www.utwente.nl/mesaplus/."

[129] L. Vogelaar, W. Nijdam, H. A. G. M. Wolferen, R. M. Ridder, F. B. Segerink, E. Flück, L. Kuipers, and N. F. Hulst, "Large area photonic crystal slabs for visible light with waveguiding defect structures: Fabrication with focused ion beam assisted laser interference lithography," Advanced Materials, vol. 13, pp. 1551-1554, 2001.

[130] R. Luttge, H. Van Wolferen, and L. Abelmann, "Laser interferometric nanolithography using a new positive chemical amplified resist," Journal of Vacuum Science \& Technology B: Microelectronics and nanometer structures, vol. 25, pp. 2476-2480, 2007.

[131] N. Maluf and K. Williams, "An Introduction to Microelectromechanical Systems Engineering," Second edition ed Norwood, MA: Artech House, Inc., 2004.

[132] V. L. Nickolay, J. S. Michael, and G. D. Panos, "Cantilever transducers as a platform for chemical and biological sensors," Review of Scientific Instruments, vol. 75, pp. 2229-2253, 2004.

[133] X. D. Yan, H. F. Ji, and T. Thundat, "Microcantilever (MCL) biosensing," Current Analytical Chemistry, vol. 2, pp. 297-307, 2006.

[134] P. Gorelkin, G. Kiselev, D. Mukhin, T. Kim, S. Kim, S. Lee, and I. Yaminskii, "Use of Biospecific Reactions for the Design of HighSensitivity Biosensors Based on Nanomechanical Cantilever Systems," Polymer Science Series a, pp. 1023-1033, 2010.

[135] M. Y. Al Aioubi, V. Djakov, S. E. Huq, and P. D. Prewett, "Deflection and load characterisation of bimorph actuators for bioMEMS and 
other applications," Microelectronic Engineering, vol. 73-74, pp. 898903, 2004.

[136] M. Alvarez and L. M. Lechuga, "Microcantilever-based platforms as biosensing tools," Analyst, vol. 135, pp. 827-836, 2010.

[137] R. Raiteri, M. Grattarola, H.-J. Butt, and P. Skládal, "Micromechanical cantilever-based biosensors," Sensors and Actuators B: Chemical, vol. 79, pp. 115-126, 2001.

[138] K. Zinoviev, C. Dominguez, J. A. Plaza, V. J. C. Busto, and L. M. Lechuga, "A novel optical waveguide microcantilever sensor for the detection of nanomechanical forces," Lightwave Technology, Journal of, vol. 24, pp. 2132-2138, 2006.

[139] M. Nordstrom, D. A. Zauner, M. Calleja, J. Hubner, and A. Boisen, "Integrated optical readout for miniaturization of cantilever-based sensor system," Applied Physics Letters, vol. 91, p. 3, 2007.

[140] M. Nordstrom, S. Keller, M. Lillemose, A. Johansson, S. r. Dohn, D. Haefliger, G. Blagoi, M. Havsteen-Jakobsen, and A. Boisen, "SU-8 Cantilevers for Bio/chemical Sensing; Fabrication, Characterisation and Development of Novel Read-out Methods," Sensors, vol. 8, pp. 1595-1612, 2008.

[141] J. W. Noh, R. Anderson, S. Kim, J. Cardenas, and G. P. Nordin, "Inplane photonic transduction of silicon-on-insulator microcantilevers," Opt. Express, vol. 16, pp. 12114-12123, 2008.

[142] S. T. Koev, R. Fernandes, W. E. Bentley, and R. Ghodssi, "A cantilever sensor with an integrated optical readout for detection of enzymatically produced homocysteine," Biomedical Circuits and Systems, IEEE Transactions on, vol. 3, pp. 415-423, 2009.

[143] Z. H. Chen, J. S. Jie, L. B. Luo, H. Wang, C. S. Lee, and S. T. Lee, "Applications of silicon nanowires functionalized with palladium nanoparticles in hydrogen sensors," Nanotechnology, vol. 18, p. 345502, 2007.

[144] S. U. Jen and T. C. Wu, "Young's modulus and hardness of Pd thin films," Thin Solid Films, vol. 492, pp. 166-172, 2005.

[145] K. M. Goeders, J. S. Colton, and L. A. Bottomley, "Microcantilevers: sensing chemical interactions via mechanical motion," Chemical reviews, vol. 108, pp. 522-542, 2008. 


\section{Biography}

Pham Van So was born on the $16^{\text {th }}$ of October 1981 in Long An, Vietnam. He completed his schooling from Le Quy Don high school for gifted students of Long An province in 1999. After completing the general course at Hochiminh City University of Technology within Programme de Formation d'Ingénieurs d'Excellence au Vietnam (PFIEV), a program in cooperation between Vietnam and France for training high-quality engineers, he continued the specialized course within the same PFIEV program and obtained the B.E. degree in Mechanical Engineering from Hanoi University of Technology, Vietnam, in 2004.

He received the M.Sc. degree in Advanced Materials Science and Engineering from SungKyunKwan University, South of Korea, in 2007.

Since September 2007, he has started his four-year doctorate research at the Integrated Optical MicroSystems group, MESA+ Institute for Nanotechnology, University of Twente, the Netherlands.

During his doctoral study, he won two Best Poster Awards at the $13^{\text {th }}$ Annual Symposium of the IEEE/LEOS Benelux Chapter, Enschede - the Netherlands in 2008, and the Annual Workshop on Semiconductor Advances for Future Electronics and Sensors (SAFE), Veldhoven - the Netherlands in 2009, respectively. He also won a Marie-Curie scholarship for a two-week summer school on "Highlights in Microtechnology" organized by F.S.R.M., Neuchâtel, Switzerland in June 2008. The work of his PhD project has been producing 4 journal papers: two of them published in Photonics Technology Letters and Optics Letters, one submitted and one to be submitted. His PhD dissertation entitled "Integrated Optical Sensors utilizing Slow-light Propagation in Grated-waveguide Cavities" will publicly be defended and approved by an international Craduation Committee organized by the University of Twente in June 2012.

Since October 2011, he has started working for ASML, one of the world's leading providers of lithography systems for the semiconductor industry.

\section{PHAM VAN SO}

Veldhoven, The Netherlands, May 2012 